\title{
Total Synthesis of (-)-Kopsifoline A and (+)-Kopsifoline E
}

\author{
In-Soo Myeong, Nadide Hazal Avci, and Mohammad Movassaghi* \\ Massachusetts Institute of Technology, Department of Chemistry, Cambridge, Massachusetts 02139 \\ movassag@mit.edu
}

\section{Supporting Information}

General Procedures $\quad$ S2

Materials

Instrumentation_

Positional Numbering System __ S3

$\mathrm{N}$ - $(p$-methoxybenzyl) Indole $(+)-\mathbf{1 0}$

Indole $(+)-11$

Vinylogous Urethane (-)-8_ S8

C21-Alcohol (-)-15 $\quad$ S10

(-)-Kopsifoline D (2)_S12

Table S1, ${ }^{1} \mathrm{H}$ NMR Comparison Table for (-)-Kopsifoline D (2) __ S14

Table S2, ${ }^{13}$ C NMR Comparison Table for (-)-Kopsifoline D (2) $\quad$ S15

Boronic Ester (-)-12 $\quad$ S16

C17-Alcohol (-)-16 S18

C17-Methyl Ether $(-)-7 \_$S20

C21-Alcohol (-)-17_

(+)-Kopsifoline E (3)_

Table S3, ${ }^{1} \mathrm{H}$ NMR Comparison Table for (+)-Kopsifoline E (3) __

Table S4, ${ }^{13} \mathrm{C}$ NMR Comparison Table for $(+)$-Kopsifoline E (3) $\quad$ S27

(-)-Kopsifoline A (1)_

Table S5, ${ }^{1} \mathrm{H}$ NMR Comparison Table for (-)-Kopsifoline A (1) __ _ S30

Table S6, ${ }^{13} \mathrm{C}$ NMR Comparison Table for (-)-Kopsifoline A (1) _

Computational Information $\quad$ S32

Figure S1, Comparison of atomic charges between vinylogous urethane 8 and indole 11 S32

Table S7, Optimized Cartesian Coordinates (+)-11 __ S33

Table S8, Optimized Cartesian Coordinates $(-)-8 \_$S34

Copy of ${ }^{1} \mathrm{H}$ and ${ }^{13} \mathrm{C}$ NMR Spectra __ 
General Procedures. All reactions were performed in oven-dried or flame-dried roundbottom flasks fitted with rubber septa and were conducted under positive argon pressure using standard Schlenk techniques, unless noted otherwise. Cannulae or gas-tight syringes with stainless steel needles were used to transfer air- or moisture-sensitive liquids. Where necessary (so noted), solutions were degassed by sparging with argon for a minimum of 10 min. Flash column chromatography was performed as described by Still et al. ${ }^{1}$ using granular silica gel (60- $\AA$ pore size, $40-63 \mu \mathrm{m}, 4-6 \% \mathrm{H}_{2} \mathrm{O}$ content, Zeochem). Analytical thin layer chromatography (TLC) was performed using glass plates pre-coated with $0.25 \mathrm{~mm} \mathrm{230-400}$ mesh silica gel impregnated with a fluorescent indicator $(254 \mathrm{~nm})$. TLC plates were visualized by exposure to short wave ultraviolet light $(254 \mathrm{~nm})$ and irreversibly stained by treatment with an aqueous solution of ceric ammonium molybdate (CAM) or an aqueous solution of potassium permanganate $\left(\mathrm{KMnO}_{4}\right)$ followed by heating $(\sim 1 \mathrm{~min})$ on a hot plate $\left(\sim 250^{\circ} \mathrm{C}\right)$. Organic solutions were concentrated at $30-35^{\circ} \mathrm{C}$ on rotary evaporators capable of achieving a minimum pressure of $\sim 10$ Torr.

Materials. Commercial reagents and solvents were used as received with the following exceptions: acetonitrile, dichloromethane, $N, N$-dimethylformamide, methanol, tetrahydrofuran, toluene, and triethylamine were purchased from J. T. Baker (CycletainerTM) and were purified by the method of Grubbs et al. under positive argon pressure. ${ }^{2}$ Deuterated solvents used for nuclear magnetic resonance (NMR) spectroscopy were purchased from Cambridge Isotope Laboratories, Inc. and were used as received with the exception of chloroform- $d$, which was stored over granular anhydrous potassium carbonate. The molarity of $n$-butyl lithium solutions was determined by titration against diphenylacetone $p$ tosylhydrazone ${ }^{3}$ (average of three titrations). 4-(Dimethylamino)pyridine was purchased from Chem-Impex International. All other solvents and chemicals were purchased from SigmaAldrich.

Instrumentation. Proton nuclear magnetic resonance $\left({ }^{1} \mathrm{H}\right.$ NMR) spectra were recorded with a Bruker AVANCE NEO 600 or a Bruker AVANCE NEO 500 spectrometer. Spectra were processed with MestReNova 14.1.2 using the automatic phasing and third-order polynomial baseline correction capabilities. Chemical shifts are recorded in parts per million on the $\delta$ scale and are referenced from the residual protium in the NMR solvent $\left(\mathrm{CHCl}_{3}: \delta 7.26\right.$, $\left.\mathrm{CD}_{2} \mathrm{HCN}: \delta 1.94\right) .{ }^{4}$ Data are reported as follows: chemical shift [multiplicity ( $\mathrm{s}=$ singlet, $\mathrm{d}=$ doublet, $\mathrm{t}=$ triplet, $\mathrm{q}=$ quartet, $\mathrm{m}=$ multiplet, $\mathrm{br}=$ broad), coupling constant( $\mathrm{s})$ in Hertz, integration, assignment]. Carbon-13 nuclear magnetic resonance $\left({ }^{13} \mathrm{C} \mathrm{NMR}\right)$ spectra were recorded with a Bruker AVANCE NEO 600 or a Bruker AVANCE NEO 500 spectrometer and are recorded in parts per million on the $\delta$ scale and are referenced from the carbon resonances of the solvent $\left(\mathrm{CDCl}_{3}: \delta 77.16, \mathrm{CD}_{3} \mathrm{CN}: \delta 118.26\right) .{ }^{4}$ Data are reported as follows: chemical shift (assignment). Infrared data were obtained with a Perkin-Elmer 2000 FTIR and are reported as follows: frequency of absorption $\left(\mathrm{cm}^{-1}\right)$ [intensity of absorption $(\mathrm{s}=$ strong, $\mathrm{m}$ $=$ medium, $\mathrm{w}=$ weak, $\mathrm{br}=$ broad $)]$. Optical rotations were measured on a Jasco-1010 polarimeter. High-resolution mass spectra (HRMS) were recorded on a Bruker Daltonics APEXIV 4.7 Tesla FT-ICR-MS using electrospray ionization (ESI) source, a Q-TOF LC/MS using ESI. 
Positional Numbering System. At least two numbering systems exist in the literature for the aspidosperma alkaloids. ${ }^{5,6}$ For direct comparison between structures, the numbering system shown below for (-)-kopsifoline A, (-)-Kopsifoline D and (+)-Kopsifoline E are used throughout this report. For each compound, we have also provided the systematic names following the International Union of Pure and Applied Chemistry (IUPAC) convention, which has a different positional numbering system.

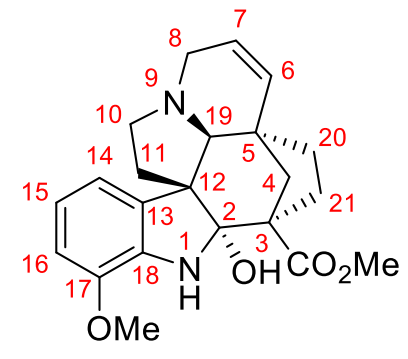

(- )-kopsifoline A (1)

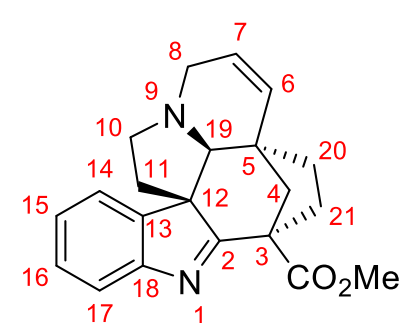

(-)-kopsifoline D (2)

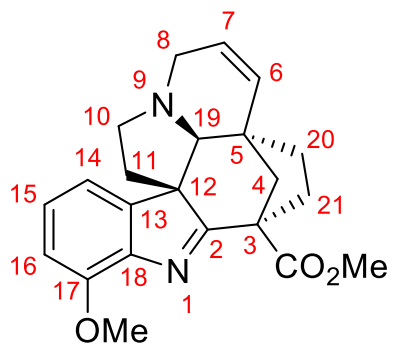

(+)-kopsifoline E (3) 


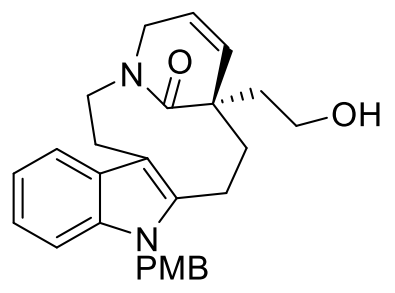

$(+)-9$

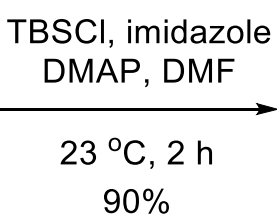

$90 \%$

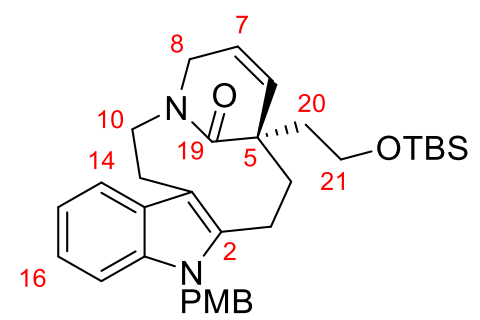

$(+)-10$

(7S)-7-(2-((tert-Butyldimethylsilyl)oxy)ethyl)-10-(4-methoxybenzyl)-1,4,7,8,9,10hexahydro-2H-3,7-methano[1] azacycloundecino[5,4-b]indol-15-one, $N$ - $(p-$ methoxybenzyl) indole $(+)-10$ :

4-(Dimethylamino)pyridine $(9.0 \mathrm{mg}, 74 \mu \mathrm{mol}, 2.0$ equiv) was added to a mixture of $(+)$ $9^{7}$ (160 mg, $0.37 \mathrm{mmol}, 1$ equiv), tert-butyldimethylsilyl chloride (84 mg, $0.55 \mathrm{mmol}, 1.5$ equiv) and imidazole (38 mg, $0.55 \mathrm{mmol}, 1.5$ equiv) in $N, N$-dimethylformamide $(2.8 \mathrm{~mL})$ at $23{ }^{\circ} \mathrm{C}$. After $2 \mathrm{~h}$, saturated aqueous sodium bicarbonate solution $(10 \mathrm{~mL})$ was added to the reaction mixture. The layers were separated, and the aqueous layer was further extracted with ethyl acetate $(2 \times 20 \mathrm{~mL})$. The combined organic extracts were further washed with saturated aqueous sodium chloride solution $(10 \mathrm{~mL})$, were dried over anhydrous sodium sulfate, were filtered, and were concentrated under reduced pressure. The residue was purified by flash column chromatography on silica gel (eluent: $20 \%$ ethyl acetate in hexanes) to afford $N$ - $(p-$ methoxybenzyl) indole (+)-10 (180 $\mathrm{mg}, 0.33 \mathrm{mmol}, 90 \%)$ as a white foam. Structural assignments were made using additional information from gCOSY, gHSQC, and gHMBC experiments.

${ }^{1} \mathrm{H}$ NMR $\left(600 \mathrm{MHz}, \mathrm{CDCl}_{3}, 25^{\circ} \mathrm{C}\right)$ :

$\delta 7.51\left(\mathrm{~d}, J=6.5 \mathrm{~Hz}, 1 \mathrm{H}, \mathrm{C}_{14} \mathbf{H}\right), 7.12-6.98(\mathrm{~m}$, $\left.3 \mathrm{H}, \mathrm{C}_{15} \mathbf{H}, \mathrm{C}_{16} \mathbf{H}, \mathrm{C}_{17} \mathbf{H}\right), 6.85(\mathrm{~d}, J=8.4 \mathrm{~Hz}, 2 \mathrm{H}$, $\left.\operatorname{Ar}_{\mathrm{PMB}} \mathbf{H}\right), 6.77\left(\mathrm{~d}, J=8.7 \mathrm{~Hz}, 2 \mathrm{H}, \operatorname{Ar}_{\mathrm{PMB}} \mathbf{H}\right)$, 5.80 (br s, 1H, C6 $\mathbf{H}), 5.49$ (d, $J=9.4 \mathrm{~Hz}, 1 \mathrm{H}$, $\left.\mathrm{C}_{7} \mathbf{H}\right), 5.30\left(\mathrm{~d}, J=17.1 \mathrm{~Hz}, 1 \mathrm{H}, \mathrm{N}_{1} \mathrm{CH}_{\mathrm{a}}\right), 5.12(\mathrm{~d}$, $J=16.7 \mathrm{~Hz}, 1 \mathrm{H}, \mathrm{N}_{1} \mathrm{CH}$ ), $4.51(\mathrm{t}, J=11.4 \mathrm{~Hz}$, $1 \mathrm{H}, \mathrm{C}_{10} \mathrm{H}_{\mathrm{a}}$ ), 4.20 (br s, $\left.1 \mathrm{H}, \mathrm{C}_{8} \mathrm{H}_{\mathrm{a}}\right) 3.90$ (br s, $1 \mathrm{H}$, $\left.\mathrm{C} 8 \mathrm{H}_{\mathrm{b}}\right), 3.73$ (s, 3H, OCH $), 3.56-3.45(\mathrm{~m}, 2 \mathrm{H}$, $\mathrm{C}_{21} \mathbf{H}_{2}$ ), 3.10 (br s, 1H, C11 $\mathbf{H}_{\mathrm{a}}$ ), 2.89 (ddd, $J=$ 14.4, 11.1, $\left.2.9 \mathrm{~Hz}, 1 \mathrm{H}, \mathrm{C}_{11} \mathrm{Hb}\right), 2.80-2.67(\mathrm{~m}$, $\left.2 \mathrm{H}, \mathrm{C}_{3} \mathrm{H}_{\mathrm{a}}, \mathrm{C}_{10} \mathrm{H}_{\mathrm{b}}\right), 2.62(\mathrm{dd}, J=15.6,9.4 \mathrm{~Hz}, 1 \mathrm{H}$, $\mathrm{C}_{3} \mathrm{Hb}$ ), 2.18 (dt, $J=13.4,7.2 \mathrm{~Hz}, 1 \mathrm{H}, \mathrm{C}_{4} \mathbf{H}_{\mathrm{a}}$ ), $2.10\left(\mathrm{dd}, J=12.7,10.5 \mathrm{~Hz}, 1 \mathrm{H}, \mathrm{C}_{20} \mathrm{H}_{\mathrm{a}}\right), 1.84$ (dd, $J=13.1,9.4 \mathrm{~Hz}, 1 \mathrm{H}, \mathrm{C}_{4} \mathbf{H b}$ ), 1.39 (ddd, $J=$ 13.2, 7.6, $\left.5.8 \mathrm{~Hz}, 1 \mathrm{H}, \mathrm{C}_{20} \mathbf{H}_{\mathrm{b}}\right), 0.83(\mathrm{~s}, 9 \mathrm{H}$, $\left.\mathrm{SiC}\left(\mathrm{CH}_{3}\right)_{3}\right), 0.00\left(\mathrm{~s}, 6 \mathrm{H}, \mathrm{Si}\left(\mathrm{CH}_{3}\right)_{2}\right)$.

${ }^{13} \mathrm{C} \mathrm{NMR}\left(150.9 \mathrm{MHz}, \mathrm{CDCl}_{3}, 25^{\circ} \mathrm{C}\right)$ :

$\delta 171.9\left(\mathbf{C}_{19}\right), 158.8\left(\mathbf{C O C H}_{3}\right), 137.1\left(\mathbf{C}_{2}\right), 136.6$ $\left(\mathbf{C}_{13}\right), 131.2\left(\mathbf{C}_{6}\right), 130.1\left(\mathrm{~N}_{1} \mathrm{CH}_{2} \mathbf{C}\right), 128.0\left(\mathbf{C}_{18}\right)$, 127.3 (Ar $\left.{ }_{\mathrm{PMB}}\right), 121.3\left(\mathbf{C}_{15}\right), 120.4\left(\mathbf{C}_{7}\right), 119.1$ $\left(\mathbf{C}_{16}\right), 117.8\left(\mathbf{C}_{14}\right), 114.2\left(\mathrm{Ar}_{\mathrm{PMB}}\right), 110.0\left(\mathbf{C}_{12}\right)$, $109.7\left(\mathbf{C}_{17}\right), 60.3\left(\mathbf{C}_{21}\right), 55.3\left(\mathrm{OCH}_{3}\right), 49.2\left(\mathbf{C}_{8}\right)$, 
$46.3\left(2 \mathrm{C}, \mathbf{C}_{10}, \mathrm{~N}_{1} \mathbf{C H}_{2}\right), 44.7\left(\mathbf{C}_{4}\right), 44.0\left(\mathbf{C}_{5}\right)$, $41.1\left(\mathbf{C}_{20}\right), 26.0\left(\mathrm{SiC}\left(\mathbf{C H}_{3}\right)_{3}\right), 22.2\left(\mathbf{C}_{11}\right), 20.0$ $\left(\mathbf{C}_{3}\right), 18.3\left(\mathrm{SiC}\left(\mathrm{CH}_{3}\right)_{3}\right),-5.2\left(\mathrm{SiC}_{\mathbf{a}} \mathrm{H}_{3}\right),-5.3$ $\left(\mathrm{SiC}_{\mathbf{b}} \mathrm{H}_{3}\right)$.

FTIR (thin film) $\mathrm{cm}^{-1}$ : 2926 (s), 1645 (m), 1512 (m), 1470 (m), 1290 (s), $1096(\mathrm{~m}), 836(\mathrm{~m}), 738(\mathrm{~m})$.

HRMS (ESI) $(\mathrm{m} / \mathrm{z})$ : calc'd for $\mathrm{C}_{33} \mathrm{H}_{45} \mathrm{~N}_{2} \mathrm{O}_{3} \mathrm{Si}[\mathrm{M}+\mathrm{H}]^{+}$: 545.3194, found: 545.3186 .

$[\alpha]_{\mathrm{D}}^{25}:$ $+45.8\left(c=0.41, \mathrm{CHCl}_{3}\right)$.

TLC (20\% ethyl acetate in hexanes), Rf: 0.50 (UV, CAM). 


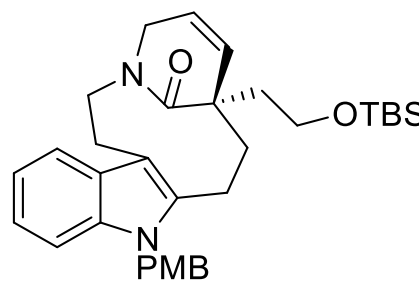

$(+)-10$

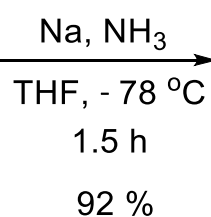

$92 \%$

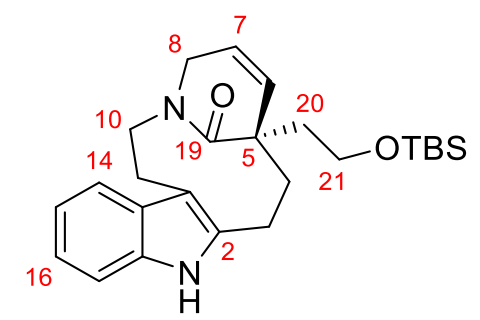

$(+)-11$

(7S)-7-(2-((tert-Butyldimethylsilyl)oxy)ethyl)-1,4,7,8,9,10-Hexahydro-2H-3,7methano[1] azacycloundecino[5,4-b]indol-15-one; indole $(+)-11$ :

A solution of the $N$-( $p$-methoxybenzyl) indole $(+)-10(180 \mathrm{mg}, 0.33 \mathrm{mmol}, 1$ equiv) in THF $(30 \mathrm{~mL})$ was added to liquid ammonia $(60 \mathrm{~mL})$ at $-78^{\circ} \mathrm{C}$. After $15 \mathrm{~min}$, sodium $(74 \mathrm{mg}$, $3.3 \mathrm{mmol}, 10$ equiv) was added to the mixture as solid, and the reaction mixture was stirred at $-78{ }^{\circ} \mathrm{C}$. After $1.5 \mathrm{~h}$, solid ammonium chloride $(1.0 \mathrm{~g})$ was added to the reaction mixture. After $5 \mathrm{~min}$, the ammonia was evaporated by slowly warming the colorless suspension to 23 ${ }^{\circ} \mathrm{C}$. After evaporation of the solvent, water $(50 \mathrm{~mL})$ and dichloromethane $(50 \mathrm{~mL})$ were added to the residue and the layers were separated. The aqueous layer was extracted with dichloromethane $(2 \times 30 \mathrm{~mL})$ and the combined organic layers were washed with saturated aqueous sodium chloride solution $(15 \mathrm{~mL})$, were dried over anhydrous sodium sulfate, were filtered, and were concentrated under reduced pressure. The resulting residue was purified by flash column chromatography on silica gel (eluent: $20 \%$ ethyl acetate in hexanes) to afford indole (+)-11 (130 mg, $0.30 \mathrm{mmol}, 92 \%)$ as a white solid. Structural assignments were made using additional information from gCOSY, gHSQC, and gHMBC experiments.

${ }^{1} \mathrm{H}$ NMR (500 MHz, $\left.\mathrm{CDCl}_{3}, 2{ }^{\circ} \mathrm{C}\right)$ :

$\delta 7.72\left(\mathrm{~s}, 1 \mathrm{H}, \mathrm{N}_{1} \mathbf{H}\right), 7.46(\mathrm{~d}, J=7.3 \mathrm{~Hz}, 1 \mathrm{H}$, $\left.\mathrm{C}_{14} \mathbf{H}\right), 7.22\left(\mathrm{~d}, J=7.6 \mathrm{~Hz}, 1 \mathrm{H}, \mathrm{C}_{17} \mathbf{H}\right), 7.09$ $7.01\left(\mathrm{~m}, 2 \mathrm{H}, \mathrm{C}_{15} \mathbf{H}, \mathrm{C}_{16} \mathbf{H}\right), 5.84(\mathrm{dt}, J=10.0,2.6$ $\left.\mathrm{Hz}, 1 \mathrm{H}, \mathrm{C}_{7} \mathbf{H}\right), 5.57$ (dt, $J=10.3,1.5 \mathrm{~Hz}, 1 \mathrm{H}$, $\mathrm{C}_{6} \mathbf{H}$ ), 4.46 (ddd, $J=13.5,11.2,2.3 \mathrm{~Hz}, 1 \mathrm{H}$, $\left.\mathrm{C}_{10} \mathbf{H}_{\mathrm{a}}\right), 4.17\left(\mathrm{~d}, J=17.3 \mathrm{~Hz}, 1 \mathrm{H}, \mathrm{C}_{8} \mathbf{H}_{\mathrm{a}}\right), 3.89(\mathrm{~d}$, $\left.J=17.6 \mathrm{~Hz}, 1 \mathrm{H}, \mathrm{C}_{8} \mathbf{H}_{\mathrm{b}}\right), 3.57-3.47(\mathrm{~m}, 2 \mathrm{H}$, $\left.\mathrm{C}_{21} \mathbf{H}\right), 3.00$ (dt, $J=15.0,2.9 \mathrm{~Hz}, 1 \mathrm{H}, \mathrm{C}_{11} \mathbf{H}_{\mathrm{a}}$ ), 2.85 (ddd, $J=14.8,11.6,2.9 \mathrm{~Hz}, 1 \mathrm{H}, \mathrm{C}_{11} \mathbf{H}_{\mathrm{b}}$ ), $2.74\left(\mathrm{dd}, J=15.3,8.2 \mathrm{~Hz}, 1 \mathrm{H}, \mathrm{C}_{3} \mathbf{H}_{\mathrm{a}}\right), 2.68(\mathrm{dt}, J$ $\left.=12.9,3.5 \mathrm{~Hz}, 1 \mathrm{H}, \mathrm{C}_{10} \mathbf{H}_{\mathrm{b}}\right), 2.63(\mathrm{dd}, J=15.3$, $10.3 \mathrm{~Hz}, 1 \mathrm{H}, \mathrm{C}_{3} \mathbf{H}_{\mathrm{b}}$ ), 2.27 (ddd, $J=13.0,8.0,6.9$ $\left.\mathrm{Hz}, 1 \mathrm{H}, \mathrm{C}_{20} \mathbf{H}_{\mathrm{a}}\right), 2.17(\mathrm{dd}, J=12.9,10.9 \mathrm{~Hz}, 1 \mathrm{H}$, $\left.\mathrm{C}_{4} \mathbf{H}_{\mathrm{a}}\right), 1.93\left(\mathrm{dd}, J=13.4,8.1 \mathrm{~Hz}, 1 \mathrm{H}, \mathrm{C}_{4} \mathbf{H}_{\mathrm{b}}\right)$, 1.47 (ddd, $J=13.4,7.8,5.7 \mathrm{~Hz}, 1 \mathrm{H}, \mathrm{C}_{20} \mathbf{H}_{\mathrm{b}}$ ), 0.84 (s, 9H, $\left.\mathrm{SiC}\left(\mathrm{CH}_{3}\right)_{3}\right),-0.02 \quad(\mathrm{~s}, \quad 6 \mathrm{H}$, $\left.\mathrm{Si}\left(\mathrm{CH}_{3}\right)_{2}\right)$.

${ }^{13} \mathrm{C} \mathrm{NMR}\left(125.8 \mathrm{MHz}, \mathrm{CDCl}_{3}, 25{ }^{\circ} \mathrm{C}\right): \quad \delta 171.8\left(\mathbf{C}_{19}\right), 135.7\left(\mathbf{C}_{13}\right), 135.6\left(\mathbf{C}_{2}\right), 131.3$ $\left(\mathbf{C}_{6}\right), 128.4\left(\mathbf{C}_{18}\right), 121.4\left(\mathbf{C}_{15}\right), 120.5\left(\mathbf{C}_{7}\right), 119.2$ $\left(\mathbf{C}_{16}\right), 117.8\left(\mathbf{C}_{14}\right), 110.5\left(\mathbf{C}_{17}\right), 109.9\left(\mathbf{C}_{12}\right), 60.3$ $\left(\mathbf{C}_{21}\right), 49.1\left(\mathbf{C}_{8}\right), 46.1\left(\mathbf{C}_{10}\right), 45.7\left(\mathbf{C}_{4}\right), 44.3\left(\mathbf{C}_{5}\right)$, 
$41.0\left(\mathbf{C}_{20}\right), 26.0\left(\mathrm{SiC}\left(\mathbf{C H}_{3}\right)_{3}\right), 21.8\left(\mathbf{C}_{3}\right), 21.7$ $\left(\mathbf{C}_{11}\right), 18.3\left(\mathrm{SiC}\left(\mathrm{CH}_{3}\right)_{3}\right),-5.2\left(\mathrm{SiC}_{\mathrm{a}} \mathrm{H}_{3}\right),-5.3$ $\left(\mathrm{SiC}_{\mathbf{b}} \mathrm{H}_{3}\right)$.

FTIR (thin film) $\mathrm{cm}^{-1}$ :

3296 (s), 2926 (m), 1632 (s), 1465 (m), 1250 (m).

HRMS (ESI) $(m / z)$ :

calc'd for $\mathrm{C}_{25} \mathrm{H}_{37} \mathrm{~N}_{2} \mathrm{O}_{2} \mathrm{Si}[\mathrm{M}+\mathrm{H}]^{+}$: 425.2619, found: 425.2622 .

$[\alpha]_{\mathrm{D}}^{25}:$

$+97.9\left(c=0.17, \mathrm{CHCl}_{3}\right)$.

TLC (30\% ethyl acetate in hexanes), $\mathrm{R} f$ :

0.35 (UV, CAM). 


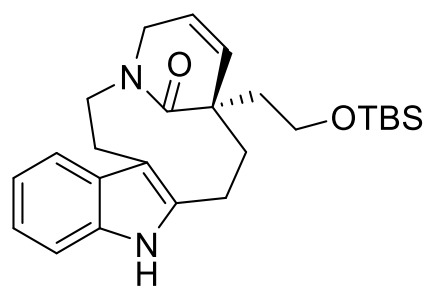

$(+)-11$
1. DIBAL, THF $0^{\circ} \mathrm{C}, 1.5 \mathrm{~h}$

2. $n$-BuLi, $\mathrm{NCCO}_{2} \mathrm{Me}$ $\mathrm{THF},-78^{\circ} \mathrm{C}, 1 \mathrm{~h}$ $80 \%$ (2 steps)

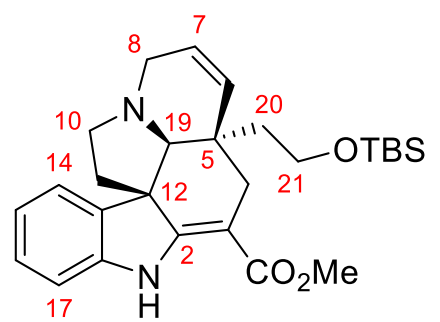

$(-)-8$

Methyl (3aS,3a $\left.{ }^{1} S, 10 \mathrm{~b} R\right)$-3a-(2-((tert-butyldimethylsilyl)oxy)ethyl)-3a,3a ${ }^{1}, 4,6,11,12$-hexa hydro-1H-indolizino $[8,1-c d]$ carbazole-5-carboxylate; vinylogous urethane $(-)-8$ :

Diisobutylaluminum hydride (1.0 $\mathrm{M}$ in hexane, $1.1 \mathrm{~mL}, 1.1 \mathrm{mmol}, 4.0$ equiv) was added slowly via syringe to a solution of indole $(+)-\mathbf{1 1}(110 \mathrm{mg}, 0.27 \mathrm{mmol}, 1$ equiv) in THF (5.4 $\mathrm{mL})$ at $0{ }^{\circ} \mathrm{C}$. After $1.5 \mathrm{~h}$, an aqueous solution of Rochelle's salt $(20 \mathrm{~mL})$ was added and the solution was stirred vigorously, and the resulting solution was allowed to warm to $23{ }^{\circ} \mathrm{C}$. Ethyl acetate $(20 \mathrm{~mL})$ was added and the layers were separated. The aqueous layer was extracted further with ethyl acetate $(2 \times 20 \mathrm{~mL})$ and the combined organic layers were dried over anhydrous sodium sulfate, were filtered through basic alumina, and were concentrated under reduced pressure. The resulting residue was used for the next reaction without further purification.

A solution of $n$-butyllithium $(1.9 \mathrm{M}, 0.43 \mathrm{~mL}, 0.81 \mathrm{mmol}, 3.0$ equiv) was added via syringe to a solution of pentacyclic imine prepared above in THF $(6.8 \mathrm{~mL})$ at $-78^{\circ} \mathrm{C}$. After $30 \mathrm{~min}$, Mander's reagent ( $86 \mu \mathrm{L}, 1.1 \mathrm{mmol}, 4.0$ equiv) was added via syringe. After $30 \mathrm{~min}$, saturated aqueous sodium bicarbonate solution $(20 \mathrm{~mL})$ was added to the reaction mixture. The layers were separated, and the aqueous layer was extracted with dichloromethane $(2 \times 20$ $\mathrm{mL}$ ). The combined organic extracts were dried over anhydrous sodium sulfate, were filtered, and were concentrated under reduced pressure. The resulting residue was purified by flash column chromatography on silica gel (eluent: $5 \%$ ethyl acetate in hexanes) to afford pentacyclic vinylogous urethane (-)-8 (100 $\mathrm{mg}, 0.22 \mathrm{mmol}, 80 \%)$ as a colorless thin film. Structural assignments were made using additional information from gCOSY, gHSQC and gHMBC experiments.

${ }^{1} \mathrm{H}$ NMR $\left(500 \mathrm{MHz}, \mathrm{CDCl}_{3}, 25^{\circ} \mathrm{C}\right)$ :

$\delta 8.98\left(\mathrm{~s}, 1 \mathrm{H}, \mathrm{N}_{1} \mathbf{H}\right), 7.22(\mathrm{~d}, J=7.3 \mathrm{~Hz}, 1 \mathrm{H}$, $\left.\mathrm{C}_{14} \mathbf{H}\right), 7.14\left(\mathrm{t}, J=7.6 \mathrm{~Hz}, 1 \mathrm{H}, \mathrm{C}_{16} \mathbf{H}\right), 6.87(\mathrm{t}, J$ $\left.=7.5 \mathrm{~Hz}, 1 \mathrm{H}, \mathrm{C}_{15} \mathbf{H}\right), 6.81(\mathrm{~d}, J=7.6 \mathrm{~Hz}, 1 \mathrm{H}$, $\left.\mathrm{C}_{17} \mathbf{H}\right), 5.82-5.73\left(\mathrm{~m}, 2 \mathrm{H}, \mathrm{C}_{6} \mathbf{H}, \mathrm{C}_{7} \mathbf{H}\right), 3.77(\mathrm{~s}$, $\left.3 \mathrm{H}, \mathrm{CO}_{2} \mathrm{CH}_{3}\right), 3.54-3.39\left(\mathrm{~m}, 3 \mathrm{H}, \mathrm{C}_{21} \mathbf{H}_{2}, \mathrm{C}_{8} \mathbf{H}_{\mathrm{a}}\right.$ ), $3.18\left(\mathrm{~d}, J=15.8 \mathrm{~Hz}, 1 \mathrm{H}, \mathrm{C}_{8} \mathbf{H}_{\mathrm{b}}\right), 3.03(\mathrm{t}, J=7.3$ $\left.\mathrm{Hz}, \quad 1 \mathrm{H}, \mathrm{C}_{10} \mathbf{H}_{\mathrm{a}}\right), 2.74-2.66\left(\mathrm{~m}, 2 \mathrm{H}, \mathrm{C}_{19} \mathbf{H}\right.$, $\left.\mathrm{C}_{10} \mathbf{H}_{\mathrm{b}}\right), 2.49\left(\mathrm{~s}, 2 \mathrm{H}, \mathrm{C}_{4} \mathbf{H}_{2}\right), 2.05(\mathrm{td}, J=11.3$, $\left.6.5 \mathrm{~Hz}, 1 \mathrm{H}, \mathrm{C}_{11} \mathbf{H}_{\mathrm{a}}\right), 1.79(\mathrm{dd}, J=11.6,4.3 \mathrm{~Hz}$, $\left.1 \mathrm{H}, \mathrm{C}_{11} \mathbf{H}_{\mathrm{b}}\right), 1.19\left(\mathrm{t}, J=7.6 \mathrm{~Hz}, 2 \mathrm{H}, \mathrm{C}_{20} \mathbf{H}_{2}\right), 0.78$ $\left(\mathrm{s}, 9 \mathrm{H}, \mathrm{SiC}\left(\mathrm{CH}_{3}\right)_{3}\right),-0.10$ to $-0.15(\mathrm{~m}, 6 \mathrm{H}$, $\left.\mathrm{Si}\left(\mathrm{CH}_{3}\right)_{2}\right)$.

${ }^{13} \mathrm{C} \mathrm{NMR}\left(125.8 \mathrm{MHz}, \mathrm{CDCl}_{3}, 25{ }^{\circ} \mathrm{C}\right): \quad \delta 169.0\left(\mathrm{OC}(\mathrm{O}) \mathrm{CH}_{3}\right), 167.0\left(\mathbf{C}_{2}\right), 143.3\left(\mathbf{C}_{18}\right)$, 
FTIR (thin film) $\mathrm{cm}^{-1}$ :

HRMS (ESI) $(m / z)$ :

$[\alpha]_{\mathrm{D}}^{25}:$

TLC (15\% ethyl acetate in hexanes), Rf:
$138.0\left(\mathbf{C}_{13}\right), 133.8\left(\mathbf{C}_{6}\right), 127.9\left(\mathbf{C}_{16}\right), 125.0\left(\mathbf{C}_{7}\right)$, $121.6\left(\mathbf{C}_{14}\right), 120.8\left(\mathbf{C}_{15}\right), 109.5\left(\mathbf{C}_{17}\right), 92.3\left(\mathbf{C}_{3}\right)$, $69.9 \quad\left(\mathbf{C}_{19}\right), \quad 59.4 \quad\left(\mathbf{C}_{21}\right), \quad 55.3 \quad\left(\mathbf{C}_{12}\right), \quad 51.2$, $\left(\mathrm{OC}(\mathrm{O}) \mathbf{C H}_{3}\right) 51.1\left(\mathbf{C}_{10}\right), 50.7\left(\mathbf{C}_{8}\right), 44.8\left(\mathbf{C}_{11}\right)$, $40.5 \quad\left(\mathbf{C}_{5}\right), \quad 37.2 \quad\left(\mathbf{C}_{20}\right), \quad 29.3 \quad\left(\mathbf{C}_{4}\right), \quad 26.1$ $\left(\mathrm{SiC}\left(\mathrm{CH}_{3}\right)_{3}\right), 18.5\left(\mathrm{SiC}\left(\mathrm{CH}_{3}\right)_{3}\right),-5.4\left(\mathrm{Si}\left(\mathrm{CH}_{3}\right)_{2}\right)$.

3370 (w), 2927 (w), 1675 (s), 1608 (s), 1464 (m), $1253(\mathrm{~m}), 1099(\mathrm{w})$.

calc'd for $\mathrm{C}_{27} \mathrm{H}_{39} \mathrm{~N}_{2} \mathrm{O}_{3} \mathrm{Si}[\mathrm{M}+\mathrm{H}]^{+}$: 467.2724, found: 467.2722 .

$-171.7\left(c=0.22, \mathrm{CHCl}_{3}\right)$.

0.32 (UV, CAM). 

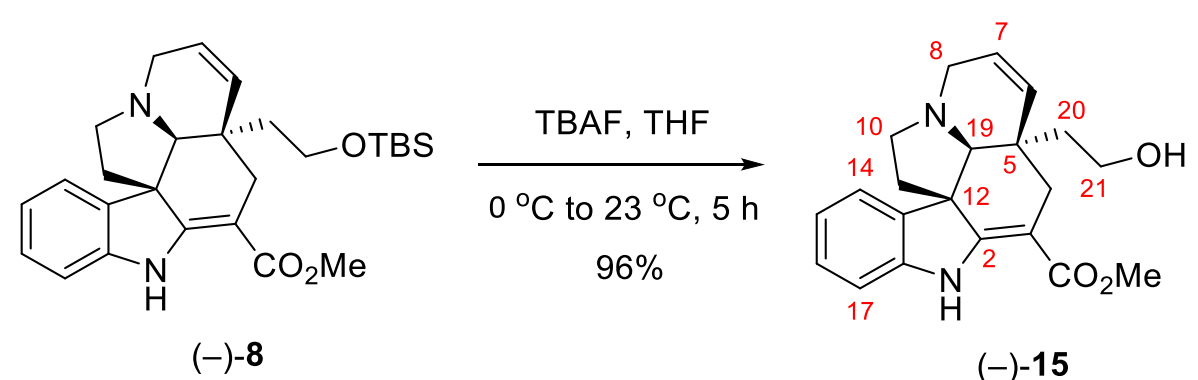

\section{Methyl (3a $\left.S, 3 a^{1} S, 10 b R\right)-3 a-(2-h y d r o x y e t h y l)-3 a, 3 a^{1}, 4,6,11,12$-hexahydro-1 $H$ - indolizino $[8,1-c d]$ carbazole-5-carboxylate; C21-alcohol (-)-15:}

Tetrabutylammonium fluoride solution (1.0 M in THF, $0.12 \mathrm{~mL}, 0.12 \mathrm{mmol}, 6.0$ equiv) was added to a solution of vinylogous urethane $(-)-8(9.4 \mathrm{mg}, 0.020 \mathrm{mmol}, 1$ equiv) in THF $(1.0 \mathrm{~mL})$ at $0{ }^{\circ} \mathrm{C}$. After the addition, the reaction mixture was allowed to $23{ }^{\circ} \mathrm{C}$. After $15 \mathrm{~h}$, saturated aqueous sodium bicarbonate solution $(10 \mathrm{~mL})$ was added to the reaction mixture and diluted with ethyl acetate $(10 \mathrm{~mL})$. The layers were separated, and the aqueous layer was extracted with ethyl acetate $(2 \times 10 \mathrm{~mL})$. The combined organic layers were dried over anhydrous sodium sulfate, were filtered, and were concentrated under reduced pressure. The resulting residue was purified by flash column chromatography on silica gel (eluent: $30 \%$ ethyl acetate in hexanes to $50 \%$ ethyl acetate in hexanes) to afford C21-alcohol (-)-15 (6.8 $\mathrm{mg}, 96 \%)$ as a colorless oil. Structural assignments were made using additional information from gCOSY, gHSQC and gHMBC experiments.

${ }^{1} \mathrm{H}$ NMR $\left(500 \mathrm{MHz}, \mathrm{CDCl}_{3}, 25^{\circ} \mathrm{C}\right)$ :

${ }^{13} \mathrm{C}$ NMR $\left(125.8 \mathrm{MHz}, \mathrm{CDCl}_{3}, 25^{\circ} \mathrm{C}\right)$ :

FTIR (thin film) $\mathrm{cm}^{-1}$ :

HRMS (ESI) $(m / z)$ : $\delta 8.96$ (br. s., $\left.1 \mathrm{H}, \mathrm{N}_{1} \mathbf{H}\right), 7.23$ (d, $J=7.3 \mathrm{~Hz}, 1 \mathrm{H}$, $\left.\mathrm{C}_{14} \mathbf{H}\right), 7.15\left(\mathrm{t}, J=7.5 \mathrm{~Hz}, 1 \mathrm{H}, \mathrm{C}_{16} \mathbf{H}\right), 6.87(\mathrm{t}, J$ $\left.=7.5 \mathrm{~Hz}, 1 \mathrm{H}, \mathrm{C}_{15} \mathbf{H}\right), 6.82(\mathrm{~d}, J=7.6 \mathrm{~Hz}, 1 \mathrm{H}$, $\left.\mathrm{C}_{17} \mathbf{H}\right), 5.82(\mathrm{ddd}, J=10.0,4.7,1.2 \mathrm{~Hz}, 1 \mathrm{H}$, $\left.\mathrm{C}_{6} \mathbf{H}\right), 5.76\left(\mathrm{dt}, J=10.0,1.8 \mathrm{~Hz}, 1 \mathrm{H}, \mathrm{C}_{7} \mathbf{H}\right), 3.77$ $\left(\mathrm{s}, 3 \mathrm{H}, \mathrm{CO}_{2} \mathrm{CH}_{3}\right), 3.56-3.43\left(\mathrm{~m}, 3 \mathrm{H}, \mathrm{C}_{21} \mathbf{H}_{2}\right.$, $\left.\mathrm{C}_{8} \mathbf{H}_{\mathrm{a}}\right), 3.20\left(\mathrm{dt}, J=16.1,1.8 \mathrm{~Hz}, 1 \mathrm{H}, \mathrm{C}_{8} \mathbf{H}_{\mathrm{b}}\right)$, $3.04\left(\mathrm{t}, J=7.2 \mathrm{~Hz}, 1 \mathrm{H}, \mathrm{C}_{10} \mathbf{H}_{\mathrm{a}}\right), 2.76(\mathrm{~s}, 1 \mathrm{H}$, $\mathrm{C}_{19} \mathbf{H}$ ), 2.71 (ddd, $J=11.0,8.4,4.7 \mathrm{~Hz}, 1 \mathrm{H}$, $\left.\mathrm{C}_{10} \mathbf{H}_{\mathrm{b}}\right), 2.55-2.46\left(\mathrm{~m}, 2 \mathrm{H}, \mathrm{C}_{4} \mathbf{H}_{2}\right), 2.07$ (td, $J=$ $\left.11.4,6.5 \mathrm{~Hz}, 1 \mathrm{H}, \mathrm{C}_{11} \mathbf{H}_{\mathrm{a}}\right), 1.80(\mathrm{dd}, J=11.4,4.1$ $\left.\mathrm{Hz}, 1 \mathrm{H}, \mathrm{C}_{11} \mathbf{H}_{\mathrm{b}}\right), 1.34-1.16\left(\mathrm{~m}, 2 \mathrm{H}, \mathrm{C}_{20} \mathbf{H}_{2}\right)$.

$\delta 169.0\left(\mathrm{OC}(\mathrm{O}) \mathrm{CH}_{3}\right), 166.7\left(\mathbf{C}_{2}\right), 143.3\left(\mathbf{C}_{18}\right)$, $137.9\left(\mathbf{C}_{13}\right), 133.5\left(\mathbf{C}_{6}\right), 128.0\left(\mathbf{C}_{16}\right), 125.6\left(\mathbf{C}_{7}\right)$, $121.6\left(\mathbf{C}_{14}\right), 120.9\left(\mathbf{C}_{15}\right), 109.6\left(\mathbf{C}_{17}\right), 92.3\left(\mathbf{C}_{3}\right)$, $69.7\left(\mathbf{C}_{19}\right), \quad 59.0 \quad\left(\mathbf{C}_{21}\right), \quad 55.3 \quad\left(\mathbf{C}_{12}\right), \quad 51.3$, $\left(\mathrm{OC}(\mathrm{O}) \mathbf{C H}_{3}\right) 51.0\left(\mathbf{C}_{10}\right), 50.6\left(\mathbf{C}_{8}\right), 44.7\left(\mathbf{C}_{11}\right)$, $40.5\left(\mathbf{C}_{5}\right), 37.5\left(\mathbf{C}_{20}\right), 29.8\left(\mathbf{C}_{4}\right)$.

3368 (br), 2924 (m), 1607 (s), 1465 (m), 1253 (m), 1156 (m).

calc'd for $\mathrm{C}_{21} \mathrm{H}_{25} \mathrm{~N}_{2} \mathrm{O}_{3}[\mathrm{M}+\mathrm{H}]^{+}$: 353.1860, found: 353.1865 . 
$[\alpha]_{\mathrm{D}}^{25}:$

$-232.0\left(c=0.20, \mathrm{CHCl}_{3}\right)$.

TLC (50\% ethyl acetate in hexanes), Rf: 0.30 (UV, CAM). 

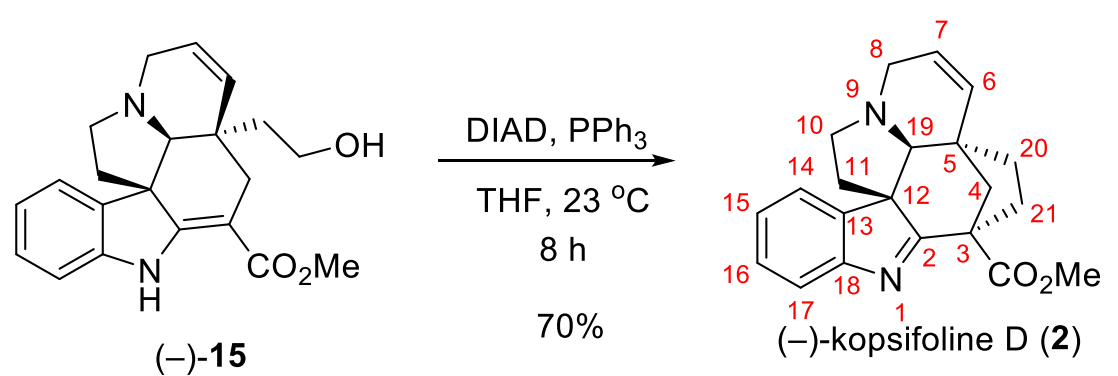

\section{(-)-Methyl (3aR,3a $\left.{ }^{1} S, 6 R, 11 \mathrm{~b} R\right)-4,5,12,13$-tetrahydro-1H,3a ${ }^{1} H, 6 H-3 a, 6-$} methanoindolizino $\left[1^{\prime}, 8^{\prime}: 2,3,4\right]$ cyclohepta $[1,2-b]$ indole-6-carboxylate; (-)-kopsifoline D (2):

Triphenylphosphine ( $36 \mathrm{mg}, 0.14 \mathrm{mmol}, 7.0$ equiv) was added to a solution of C21alcohol (-)-15 (6.9 mg, $20 \mu \mathrm{mol}, 1$ equiv) in THF $(2.0 \mathrm{~mL})$ at $23{ }^{\circ} \mathrm{C}$ as a solid. Diisopropyl azodicarboxylate $\left(20 \mu \mathrm{L}, 0.10 \mathrm{mmol}, 5.0\right.$ equiv) was added to the reaction mixture at $23{ }^{\circ} \mathrm{C}$. After $8 \mathrm{~h}$, the reaction mixture was concentrated under reduced pressure. The resulting residue was purified by flash column chromatography on silica gel (eluent: $10 \%$ ethyl acetate in hexanes with $2.5 \%$ triethyl amine). The resulting residue was purified by flash column chromatography on basic alumina (eluent: $20 \%$ ethyl acetate in hexanes) to afford (-)kopsifoline D (2, $4.6 \mathrm{mg}, 14 \mu \mathrm{mol}, 70 \%)$ as a white solid. Structural assignments were made using additional information from gCOSY, gHSQC and gHMBC experiments.

${ }^{1} \mathrm{H}$ NMR $\left(600 \mathrm{MHz}, \mathrm{CDCl}_{3}, 25^{\circ} \mathrm{C}\right)$ :

${ }^{13} \mathrm{C}$ NMR $\left(150.9 \mathrm{MHz}, \mathrm{CDCl}_{3}, 25^{\circ} \mathrm{C}\right)$ : $\delta 7.57\left(\mathrm{~d}, J=7.6 \mathrm{~Hz}, 1 \mathrm{H}, \mathrm{C}_{17} \mathbf{H}\right), 7.47(\mathrm{~d}, J=7.0$ $\left.\mathrm{Hz}, 1 \mathrm{H}, \mathrm{C}_{14} \mathbf{H}\right), 7.30(\mathrm{td}, J=7.6,1.2 \mathrm{~Hz}, 1 \mathrm{H}$, $\left.\mathrm{C}_{15} \mathbf{H}\right), 7.19\left(\mathrm{td}, J=7.5,0.9 \mathrm{~Hz}, 1 \mathrm{H}, \mathrm{C}_{16} \mathbf{H}\right), 5.76$ (ddd, $\left.J=10.0,4.4,1.8 \mathrm{~Hz}, 1 \mathrm{H}, \mathrm{C}_{7} \mathbf{H}\right), 5.63$ (dt, $J$ $\left.=10.0,1.8 \mathrm{~Hz}, 1 \mathrm{H}, \mathrm{C}_{6} \mathbf{H}\right), 3.79\left(\mathrm{~s}, 3 \mathrm{H}, \mathrm{CO}_{2} \mathrm{CH}_{3}\right)$, 3.58 (dt, $J=17.6,1.8 \mathrm{~Hz}, 1 \mathrm{H}, \mathrm{C}_{8} \mathbf{H}_{\mathrm{a}}$ ), 3.42 (ddd, $\left.J=17.8,4.3,1.2 \mathrm{~Hz}, 1 \mathrm{H}, \mathrm{C}_{8} \mathbf{H}_{\mathrm{b}}\right), 3.35(\mathrm{td}, J=$ $\left.8.2,6.2 \mathrm{~Hz}, 1 \mathrm{H}, \mathrm{C}_{10} \mathbf{H}_{\mathrm{a}}\right), 3.29(\mathrm{td}, J=7.8,5.6 \mathrm{~Hz}$, $\left.1 \mathrm{H}, \mathrm{C}_{10} \mathbf{H}_{\mathrm{b}}\right), 2.77$ (br s, $\left.1 \mathrm{H}, \mathrm{C}_{19} \mathbf{H}\right), 2.65-2.57(\mathrm{~m}$, $1 \mathrm{H}, \mathrm{C}_{11} \mathbf{H}_{\mathrm{a}}$ ), 2.63 (br d, $J=11.7 \mathrm{~Hz}, 1 \mathrm{H}, \mathrm{C}_{4} \mathbf{H}_{\mathrm{a}}$ ), $2.43\left(\mathrm{td}, J=12.0,8.4 \mathrm{~Hz}, 1 \mathrm{H}, \mathrm{C}_{21} \mathbf{H}_{\mathrm{a}}\right), 2.17-2.04$ $\left(\mathrm{m}, 2 \mathrm{H}, \mathrm{C}_{11} \mathbf{H}_{\mathrm{b}}, \mathrm{C}_{21} \mathbf{H}_{\mathrm{b}}\right), 1.77(\mathrm{~d}, J=12.3,1.8$ $\mathrm{Hz}, 1 \mathrm{H}, \mathrm{C}_{4} \mathbf{H}_{\mathrm{b}}$ ), 1.69 (ddd, $J=14.4,12.0,2.3 \mathrm{~Hz}$, $\left.1 \mathrm{H}, \mathrm{C}_{20} \mathbf{H}_{\mathrm{a}}\right), 1.22-1.14\left(\mathrm{~m}, 1 \mathrm{H}, \mathrm{C}_{20} \mathbf{H}_{\mathrm{b}}\right)$.

$\delta 188.5\left(\mathbf{C}_{2}\right), 172.5\left(\mathrm{OC}(\mathrm{O}) \mathrm{CH}_{3}\right), 154.6\left(\mathbf{C}_{18}\right)$, $147.4\left(\mathbf{C}_{13}\right), 132.6\left(\mathbf{C}_{6}\right), 127.7\left(\mathbf{C}_{16}\right), 126.1$ $\left(\mathbf{C}_{15}\right), 125.5\left(\mathbf{C}_{7}\right), 121.6\left(\mathbf{C}_{14}\right), 120.5\left(\mathbf{C}_{17}\right), 69.3$ $\left(\mathbf{C}_{19}\right), 62.8\left(\mathbf{C}_{12}\right), 56.7\left(\mathbf{C}_{3}\right), 52.8,\left(\mathrm{OC}(\mathrm{O}) \mathbf{C H}_{3}\right)$ $51.1\left(\mathbf{C}_{10}\right), 47.0\left(\mathbf{C}_{8}\right), 43.6\left(\mathbf{C}_{4}\right), 42.5\left(\mathbf{C}_{5}\right), 39.2$ $\left(\mathbf{C}_{21}\right) 35.1\left(\mathbf{C}_{20}\right), 35.0\left(\mathbf{C}_{11}\right)$.

FTIR (thin film) $\mathrm{cm}^{-1}$ : 3303 (br), 2981 (w), 2924 (w), 1730 (s), 1466 (w), $1238(\mathrm{~m}), 1109(\mathrm{~m})$.

calc'd for $\mathrm{C}_{21} \mathrm{H}_{23} \mathrm{~N}_{2} \mathrm{O}_{2}[\mathrm{M}+\mathrm{H}]^{+}$: 335.1754 , 
found: 335.1758 .

$[\alpha]_{\mathrm{D}}^{25}:$

$-87.9\left(c=0.10, \mathrm{CHCl}_{3}\right){ }^{6}$

TLC (50\% ethyl acetate in hexanes), Rf: 0.40 (UV, CAM). 
Table S1. Comparison of ${ }^{1} \mathrm{H}$ NMR data of (-)-kopsifoline D (2) with literature data

\begin{tabular}{|c|c|c|c|c|}
\hline Assignment & $\begin{array}{l}\text { Naturally occurring } \\
\text { (-)-kopsifoline D } \\
\left(400 \mathrm{MHz}, \mathrm{CDCl}_{3}\right)^{8}\end{array}$ & 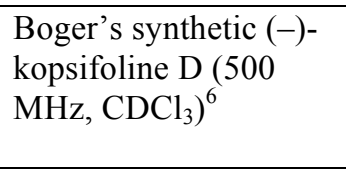 & $\begin{array}{l}\text { Peng's synthetic (-)- } \\
\text { kopsifoline } \mathrm{D}(400 \\
\left.\mathrm{MHz}, \mathrm{CDCl}_{3}\right)^{9}\end{array}$ & 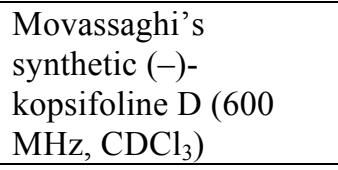 \\
\hline N1-H & - & - & - & - \\
\hline $\mathrm{C} 2$ & - & - & - & - \\
\hline $\mathrm{C} 3$ & - & - & - & 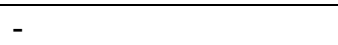 \\
\hline $\mathrm{C} 4$ & $\begin{array}{l}1.78(\mathrm{dd}, J=13,2 \\
\mathrm{Hz}, 1 \mathrm{H}) \\
2.63(\mathrm{br} \mathrm{d}, J=13 \mathrm{~Hz}, \\
1 \mathrm{H})\end{array}$ & $\begin{array}{l}1.77(\mathrm{~d}, J=12.3 \mathrm{~Hz}, \\
1 \mathrm{H}) \\
2.63(\mathrm{br} \mathrm{d}, J=11.7 \\
\mathrm{Hz}, 1 \mathrm{H})\end{array}$ & $\begin{array}{l}1.77(\mathrm{dd}, J=12.4,1.8 \\
\mathrm{Hz}, 1 \mathrm{H}) \\
2.63(\mathrm{~d}, J=10.5 \mathrm{~Hz} \\
1 \mathrm{H})\end{array}$ & $\begin{array}{l}1.77(\mathrm{dd}, J=12.3,1.8 \\
\mathrm{Hz}, 1 \mathrm{H}) \\
2.63(\mathrm{br} \mathrm{d}, J=11.7 \\
\mathrm{Hz}, 1 \mathrm{H})\end{array}$ \\
\hline $\mathrm{C} 5$ & - & 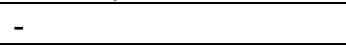 & - & 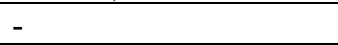 \\
\hline C6 & $\begin{array}{l}5.64(\mathrm{dt}, J=10,2 \mathrm{~Hz}, \\
1 \mathrm{H})\end{array}$ & $\begin{array}{l}5.63(\mathrm{~d}, J=10.2 \mathrm{~Hz}, \\
1 \mathrm{H})\end{array}$ & $\begin{array}{l}5.63(\mathrm{dt}, J=10.1,2.0 \\
\mathrm{Hz}, 1 \mathrm{H})\end{array}$ & $\begin{array}{l}5.63(\mathrm{dt}, J=10.0,1.8 \\
\mathrm{Hz}, 1 \mathrm{H})\end{array}$ \\
\hline $\mathrm{C} 7$ & $\begin{array}{l}5.77(\mathrm{ddd}, J=10,4,2 \\
\mathrm{Hz}, 1 \mathrm{H})\end{array}$ & $\begin{array}{l}5.76(\mathrm{dd}, J=10.2,4.2 \\
\mathrm{Hz}, 1 \mathrm{H})\end{array}$ & $\begin{array}{l}5.76(\mathrm{ddd}, J=10.1, \\
4.3,1.7 \mathrm{~Hz}, 1 \mathrm{H})\end{array}$ & $\begin{array}{l}5.76(\mathrm{ddd}, J=10.0, \\
4.4,1.8 \mathrm{~Hz}, 1 \mathrm{H})\end{array}$ \\
\hline C8 & $\begin{array}{l}3.42(\mathrm{dd}, J=17,4 \\
\mathrm{Hz}, 1 \mathrm{H}) \\
3.59(\mathrm{br} \mathrm{d}, J=17 \mathrm{~Hz} \\
1 \mathrm{H})\end{array}$ & $\begin{array}{l}3.41(\mathrm{dd}, J=17.7,4.4 \\
\mathrm{Hz}, 1 \mathrm{H}) \\
3.58(\mathrm{~d}, J=17.6 \mathrm{~Hz} \\
1 \mathrm{H})\end{array}$ & $\begin{array}{l}3.41(\mathrm{ddd}, J=18.0 \\
4.5,1.7 \mathrm{~Hz}, 1 \mathrm{H}) \\
3.58(\mathrm{dt}, J=17.7,2.1 \\
\mathrm{Hz}, 1 \mathrm{H})\end{array}$ & $\begin{array}{l}3.41(\mathrm{ddd}, J=17.8, \\
4.3,1.2 \mathrm{~Hz}, 1 \mathrm{H}) \\
3.58(\mathrm{dt}, J=17.6,1.8 \\
\mathrm{Hz}, 1 \mathrm{H})\end{array}$ \\
\hline $\mathrm{C} 10$ & $\begin{array}{l}3.31(\mathrm{~m}, 1 \mathrm{H}) \\
3.36(\mathrm{td}, J=8,6 \mathrm{~Hz} \\
1 \mathrm{H})\end{array}$ & $\begin{array}{l}3.32-3.25(\mathrm{~m}, 1 \mathrm{H}) \\
3.35(\mathrm{q}, J=7.8 \mathrm{~Hz} \\
1 \mathrm{H})\end{array}$ & $\begin{array}{l}3.32-3.25(\mathrm{~m}, 1 \mathrm{H}) \\
3.35(\mathrm{td}, J=8.4,6.0 \\
\mathrm{Hz}, 1 \mathrm{H})\end{array}$ & $\begin{array}{l}3.29(\mathrm{td}, J=7.8,5.6 \\
\mathrm{Hz}, 1 \mathrm{H}) \\
3.35(\mathrm{td}, J=8.2,6.2 \\
\mathrm{Hz}, 1 \mathrm{H})\end{array}$ \\
\hline C11 & & $\begin{array}{l}2.18-2.03(\mathrm{~m}, 1 \mathrm{H}) \\
2.65-2.56(\mathrm{~m}, 1 \mathrm{H})\end{array}$ & & $\begin{array}{l}2.17-2.04(\mathrm{~m}, 1 \mathrm{H}) \\
2.65-2.57(\mathrm{~m}, 1 \mathrm{H})\end{array}$ \\
\hline $\mathrm{C} 12$ & - & - & - & \\
\hline $\mathrm{C} 13$ & - & - & - & 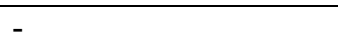 \\
\hline C14 & $\begin{array}{l}7.48(\text { br d, } J=8 \mathrm{~Hz}, \\
1 \mathrm{H})\end{array}$ & $\begin{array}{l}7.47(\mathrm{~d}, J=7.3 \mathrm{~Hz}, \\
1 \mathrm{H})\end{array}$ & $\begin{array}{l}7.47(\mathrm{~d}, J=7.3 \mathrm{~Hz}, \\
1 \mathrm{H})\end{array}$ & $\begin{array}{l}7.47(\mathrm{~d}, J=7.0 \mathrm{~Hz}, \\
1 \mathrm{H})\end{array}$ \\
\hline $\mathrm{C} 15$ & $\begin{array}{l}7.30(\mathrm{td}, J=8,2 \mathrm{~Hz}, \\
1 \mathrm{H})\end{array}$ & $\begin{array}{l}7.29(\mathrm{t}, J=7.6 \mathrm{~Hz}, \\
1 \mathrm{H})\end{array}$ & $\begin{array}{l}7.30(\mathrm{td}, J=7.6,1.3 \\
\mathrm{Hz}, 1 \mathrm{H})\end{array}$ & $\begin{array}{l}7.30(\mathrm{td}, J=7.6,1.2 \\
\mathrm{Hz}, 1 \mathrm{H})\end{array}$ \\
\hline C16 & $\begin{array}{l}7.20(\mathrm{td}, J=8,2 \mathrm{~Hz}, \\
1 \mathrm{H})\end{array}$ & $\begin{array}{l}7.19(\mathrm{t}, J=7.4 \mathrm{~Hz}, \\
1 \mathrm{H})\end{array}$ & $\begin{array}{l}7.19(\mathrm{td}, J=7.4,1.1 \\
\mathrm{Hz}, 1 \mathrm{H})\end{array}$ & $\begin{array}{l}7.19(\mathrm{td}, J=7.5,0.9 \\
\mathrm{Hz}, 1 \mathrm{H})\end{array}$ \\
\hline $\mathrm{C} 17$ & $\begin{array}{l}7.57(\mathrm{dd}, J=8,2 \mathrm{~Hz}, \\
1 \mathrm{H})\end{array}$ & $\begin{array}{l}7.57(\mathrm{~d}, J=7.7 \mathrm{~Hz}, \\
1 \mathrm{H})\end{array}$ & $\begin{array}{l}7.57(\mathrm{~d}, J=7.7 \mathrm{~Hz}, \\
1 \mathrm{H})\end{array}$ & $\begin{array}{l}7.57(\mathrm{~d}, J=7.6 \mathrm{~Hz}, \\
1 \mathrm{H})\end{array}$ \\
\hline $\mathrm{C} 18$ & - & -1 & - & - \\
\hline C19 & $2.78(\mathrm{br} \mathrm{s}, 1 \mathrm{H})$ & $2.77(\mathrm{~s}, 1 \mathrm{H})$ & $\begin{array}{l}2.77(\mathrm{~d}, J=1.7 \mathrm{~Hz} \\
1 \mathrm{H})\end{array}$ & $2.77($ br s, $1 \mathrm{H})$ \\
\hline $\mathrm{C} 20$ & $\begin{array}{l}1.20(\mathrm{~m}, 1 \mathrm{H}) \\
1.69(\mathrm{~m}, 1 \mathrm{H})\end{array}$ & $\begin{array}{l}1.23-1.14(\mathrm{~m}, 1 \mathrm{H}) \\
1.69(\mathrm{t}, J=13.1 \mathrm{~Hz}, \\
1 \mathrm{H})\end{array}$ & $\begin{array}{l}1.23-1.14(\mathrm{~m}, 1 \mathrm{H}) \\
1.69(\mathrm{ddd}, J=14.3, \\
11.8,2.5 \mathrm{~Hz}, 1 \mathrm{H})\end{array}$ & $\begin{array}{l}1.22-1.14(\mathrm{~m}, 1 \mathrm{H}) \\
1.69(\mathrm{ddd}, J=14.4, \\
12.0,2.3 \mathrm{~Hz}, 1 \mathrm{H})\end{array}$ \\
\hline $\mathrm{C} 21$ & $\begin{array}{l}2.13(\mathrm{dddd}, J=12,9 \\
3,2 \mathrm{~Hz}, 1 \mathrm{H}) \\
2.43(\mathrm{td}, J=12,8 \mathrm{~Hz}, \\
1 \mathrm{H})\end{array}$ & $\begin{array}{l}2.18-2.03(\mathrm{~m}, 1 \mathrm{H}) \\
2.43(\mathrm{td}, J=11.9,8.2 \\
\mathrm{Hz}, 1 \mathrm{H})\end{array}$ & $\begin{array}{l}2.18-2.03(\mathrm{~m}, 1 \mathrm{H}) \\
2.43(\mathrm{td}, J=12.1,8.5 \\
\mathrm{Hz}, 1 \mathrm{H})\end{array}$ & $\begin{array}{l}2.17-2.04(\mathrm{~m}, 1 \mathrm{H}) \\
2.43(\mathrm{td}, J=12.0,8.4 \\
\mathrm{Hz}, 1 \mathrm{H})\end{array}$ \\
\hline $\mathrm{CO}_{2} \mathrm{CH}_{3}$ & $3.79(\mathrm{~s}, 3 \mathrm{H})$ & $3.79(\mathrm{~s}, 3 \mathrm{H})$ & $3.79(\mathrm{~s}, 3 \mathrm{H})$ & $3.79(\mathrm{~s}, 3 \mathrm{H})$ \\
\hline
\end{tabular}


Table S2. Comparison of ${ }^{13} \mathrm{C}$ NMR data of (-)-kopsifoline D (2) with literature data

\begin{tabular}{|c|c|c|c|c|c|c|c|}
\hline \multirow[t]{2}{*}{$\begin{array}{l}\text { Assignment } \\
\\
\mathrm{C} 2\end{array}$} & \multicolumn{2}{|c|}{$\begin{array}{l}\text { Naturally occurring }(-)- \\
\text { kopsifoline D }(100 \mathrm{MHz} \text {, } \\
\left.\mathrm{CDCl}_{3}\right),{ }^{8} \text { Chemical Shift } \\
\text { Difference } \\
\Delta=\delta \text { (Movassaghi's synthetic, } \\
\text { solvent ref. } \delta 77.16)-\delta \text { (naturally } \\
\text { occurring) }\end{array}$} & \multicolumn{2}{|c|}{$\begin{array}{l}\text { Boger's synthetic (-)- } \\
\text { kopsifoline D (150 } \\
\left.\mathrm{MHz}, \mathrm{CDCl}_{3}\right){ }^{6} \\
\text { Chemical Shift } \\
\text { Difference }^{11} \\
\Delta=\delta \text { (Movassaghi's synthetic, } \\
\text { solvent ref. } \delta 77.16)-\delta \\
\text { (Boger's synthetic) } \\
\end{array}$} & \multicolumn{2}{|c|}{$\begin{array}{l}\text { Peng's synthetic (-)- } \\
\text { kopsifoline D }(125 \\
\left.\mathrm{MHz} \mathrm{CDCl}_{3}\right),{ }^{9} \\
\text { Chemical Shift } \\
\text { Difference } \\
\Delta=\delta \text { (Movassaghi's synthetic, } \\
\text { solvent ref. } \delta 77.16)-\delta \text { (Peng's } \\
\text { synthetic) }\end{array}$} & \multirow[t]{2}{*}{$\begin{array}{l}\text { Movassaghi's } \\
\text { synthetic (-)- } \\
\text { kopsifoline D } \\
\left(150 \mathrm{MHz}^{-}\right. \\
\left.\mathrm{CDCl}_{3}\right) \\
188.5\end{array}$} \\
\hline & 187.7 & $0.8^{12}$ & 188.3 & 0.2 & 188.3 & 0.2 & \\
\hline $\mathrm{C} 3$ & 56.5 & 0.2 & 56.5 & 0.2 & 56.6 & 0.1 & 56.7 \\
\hline $\mathrm{C} 4$ & 43.4 & 0.2 & 43.4 & 0.2 & 43.4 & 0.2 & 43.6 \\
\hline $\mathrm{C} 5$ & 42.3 & 0.2 & 42.3 & 0.2 & 42.4 & 0.1 & 42.5 \\
\hline C6 & 132.5 & 0.1 & 132.5 & 0.1 & 132.5 & 0.1 & 132.6 \\
\hline $\mathrm{C} 7$ & 125.2 & 0.3 & 125.3 & 0.2 & 125.4 & 0.1 & 125.5 \\
\hline $\mathrm{C} 8$ & 46.9 & 0.1 & 46.9 & 0.1 & 46.9 & 0.1 & 47.0 \\
\hline $\mathrm{C} 10$ & 50.9 & 0.2 & 50.9 & 0.2 & 50.9 & 0.2 & 51.1 \\
\hline $\mathrm{C} 11$ & 34.7 & 0.3 & 34.8 & 0.2 & 34.8 & 0.2 & 35.0 \\
\hline $\mathrm{C} 12$ & 63.0 & -0.2 & 62.7 & 0.1 & 62.7 & 0.1 & 62.8 \\
\hline $\mathrm{C} 13$ & 147.0 & 0.4 & 147.2 & 0.2 & 147.2 & 0.2 & 147.4 \\
\hline C14 & 121.6 & 0.0 & 121.5 & 0.1 & 121.5 & 0.1 & 121.6 \\
\hline $\mathrm{C} 15$ & 125.8 & 0.3 & 125.9 & 0.2 & 125.9 & 0.2 & 126.1 \\
\hline C16 & 127.5 & 0.2 & 127.5 & 0.2 & 127.5 & 0.2 & 127.7 \\
\hline $\mathrm{C} 17$ & 120.3 & 0.2 & 120.3 & 0.2 & 120.4 & 0.2 & 120.5 \\
\hline $\mathrm{C} 18$ & 154.3 & 0.3 & 154.4 & 0.2 & 154.4 & 0.2 & 154.6 \\
\hline C19 & 69.1 & 0.2 & 69.1 & 0.2 & 69.1 & 0.2 & 69.3 \\
\hline $\mathrm{C} 20$ & 35.0 & 0.1 & 35.0 & 0.1 & 35.0 & 0.1 & 35.1 \\
\hline $\mathrm{C} 21$ & 39.0 & 0.2 & 39.0 & 0.2 & 39.0 & 0.2 & 39.2 \\
\hline $\mathrm{CO}_{2} \mathrm{CH}_{3}$ & 52.6 & 0.2 & 52.6 & 0.2 & 52.6 & 0.2 & 52.8 \\
\hline $\mathrm{CO}_{2} \mathrm{CH}_{3}$ & 172.2 & 0.3 & 172.4 & 0.1 & 172.4 & 0.1 & 172.5 \\
\hline
\end{tabular}




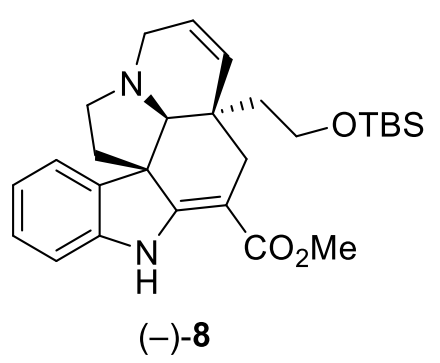

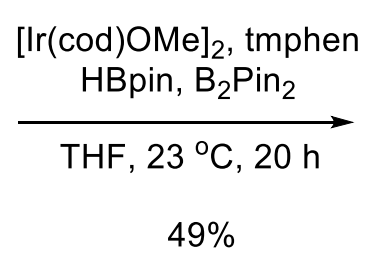

$49 \%$

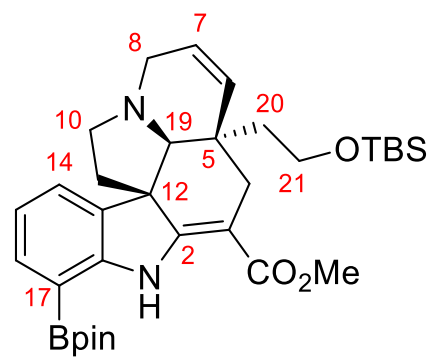

$(-)-12$

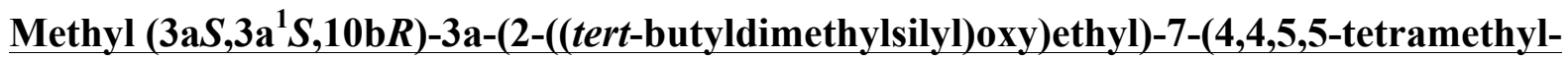
1,3,2-dioxaborolan-2-yl)-3a,3a ${ }^{1}, 4,6,11,12$-hexahydro- $1 H$-indolizino $[8,1$-cd $]$ carbazole-5carboxylate; boronic ester $(-)-12$ :

Pinacoleborane (75 $\mu \mathrm{L}, 0.52 \mathrm{mmol}, 5.0$ equiv) and Bis(pinacolato)diboron (130 mg, $0.52 \mathrm{mmol}, 5.0$ equiv) were added to a solution of (1,5-cyclooctadiene)(methoxy)iridium(I) dimer $(6.8 \mathrm{mg}, 10 \mu \mathrm{mol}, 10 \mathrm{~mol} \%)$ and 3,4,7,8-tetramethyl-1,10-phenanthroline $(4.9 \mathrm{mg}, 21$ $\mu \mathrm{mol}, 20 \mathrm{~mol} \%)$ in THF $(0.20 \mathrm{~mL})$ at $23{ }^{\circ} \mathrm{C}$. A solution of vinylogous urethane $(-)-8(48 \mathrm{mg}$, $0.10 \mathrm{mmol}, 1$ equiv) in THF $(0.80 \mathrm{~mL})$ was added to the reaction mixture at $23^{\circ} \mathrm{C}$. After $20 \mathrm{~h}$, the reaction mixture was concentrated under reduced pressure. The resulting residue was purified by flash column chromatography on silica gel (eluent: 5\% ethyl acetate in hexanes to $10 \%$ ethyl acetate in hexanes) to afford boronic ester (-)-12 (29 $\mathrm{mg}, 0.26 \mathrm{mmol}, 49 \%)$ as a colorless oil. Structural assignments were made using additional information from gCOSY, gHSQC and gHMBC experiments.

${ }^{1} \mathrm{H}$ NMR $\left(500 \mathrm{MHz}, \mathrm{CDCl}_{3}, 25^{\circ} \mathrm{C}\right)$ :

$\delta 9.53$ (br. s., $\left.1 \mathrm{H}, \mathrm{N}_{1} \mathbf{H}\right), 7.50(\mathrm{~d}, J=7.6 \mathrm{~Hz}, 1 \mathrm{H}$, $\left.\mathrm{C}_{16} \mathbf{H}\right), 7.28\left(\mathrm{~d}, J=7.3 \mathrm{~Hz}, 1 \mathrm{H}, \mathrm{C}_{14} \mathbf{H}\right), 6.83(\mathrm{t}, J$ $\left.=7.3 \mathrm{~Hz}, 1 \mathrm{H}, \mathrm{C}_{15} \mathbf{H}\right), 5.83-5.71\left(\mathrm{~m}, 2 \mathrm{H}, \mathrm{C}_{6} \mathbf{H}\right.$, $\left.\mathrm{C}_{7} \mathbf{H}\right), 3.82\left(\mathrm{~s}, 3 \mathrm{H}, \mathrm{CO}_{2} \mathrm{CH}_{3}\right), 3.52(\mathrm{td}, J=10.0$, $\left.6.7 \mathrm{~Hz}, 1 \mathrm{H}, \mathrm{C}_{21} \mathbf{H}_{\mathrm{a}}\right), 3.48-3.39\left(\mathrm{~m}, 2 \mathrm{H}, \mathrm{C}_{21} \mathbf{H}_{\mathrm{b}}\right.$, $\left.\mathrm{C}_{8} \mathbf{H}_{\mathrm{a}}\right), 3.19\left(\mathrm{~d}, J=15.6 \mathrm{~Hz}, 1 \mathrm{H}, \mathrm{C}_{8} \mathbf{H}_{\mathrm{b}}\right), 3.03(\mathrm{t}$, $\left.J=6.7 \mathrm{~Hz}, 1 \mathrm{H}, \mathrm{C}_{10} \mathbf{H}_{\mathrm{a}}\right), 2.77-2.65(\mathrm{~m}, 2 \mathrm{H}$, $\left.\mathrm{C}_{10} \mathbf{H}_{\mathrm{b}}, \mathrm{C}_{19} \mathbf{H}\right), 2.57-2.44\left(\mathrm{~m}, 2 \mathrm{H}, \mathrm{C}_{4} \mathbf{H}_{2}\right), 2.10$ $1.99\left(\mathrm{~m}, 1 \mathrm{H}, \mathrm{C}_{11} \mathbf{H}_{\mathrm{a}}\right), 1.83-1.76\left(\mathrm{~m}, 1 \mathrm{H}, \mathrm{C}_{11} \mathbf{H}_{\mathrm{b}}\right)$, $1.41\left(\mathrm{~s}, 12 \mathrm{H}, \mathrm{B}\left(\mathrm{OC}\left(\mathrm{CH}_{3}\right)_{2}\right)_{2}\right), 1.24-1.16(\mathrm{~m}, 2 \mathrm{H}$, $\left.\mathrm{C}_{20} \mathbf{H}_{2}\right), 0.77$ (s, 9H, $\left.\mathrm{SiC}\left(\mathrm{CH}_{3}\right)_{3}\right),-0.08$ to -0.14 $\left(\mathrm{m}, 6 \mathrm{H}, \mathrm{Si}\left(\mathrm{CH}_{3}\right)_{2}\right)$.

${ }^{13} \mathrm{C} \mathrm{NMR}\left(125.8 \mathrm{MHz}, \mathrm{CDCl}_{3}, 25{ }^{\circ} \mathrm{C}\right): \quad \delta 168.1\left(\mathrm{OC}(\mathrm{O}) \mathrm{CH}_{3}\right), 165.1\left(\mathbf{C}_{2}\right), 150.1\left(\mathbf{C}_{18}\right)$, $137.3\left(\mathbf{C}_{13}\right), 133.8\left(2 \mathrm{C}, \mathbf{C}_{16}, \mathbf{C}_{6}\right), 124.9\left(\mathbf{C}_{7}\right)$, $124.4\left(\mathbf{C}_{14}\right), 119.8\left(\mathbf{C}_{15}\right), 108.3\left(\mathbf{C}_{17}\right), 92.3\left(\mathbf{C}_{3}\right)$, $84.1\left(\mathrm{~B}\left(\mathrm{OC}\left(\mathrm{CH}_{3}\right)_{2}\right)_{2}\right), 70.0\left(\mathbf{C}_{19}\right), 59.5\left(\mathbf{C}_{21}\right)$, $55.2\left(\mathbf{C}_{12}\right), 51.1\left(2 \mathrm{C}, \mathrm{OC}(\mathrm{O}) \mathbf{C H}_{3}, \mathbf{C}_{10}\right), 50.6$ $\left(\mathbf{C}_{8}\right), 45.0\left(\mathbf{C}_{11}\right), 40.2\left(\mathbf{C}_{5}\right), 37.3\left(\mathbf{C}_{20}\right), 29.7\left(\mathbf{C}_{4}\right)$, $26.1\left(\mathrm{SiC}\left(\mathbf{C H}_{3}\right)_{3}\right), 25.2\left(\mathrm{~B}\left(\mathrm{OC}\left(\mathrm{CH}_{3}\right)_{2}\right)_{2}\right), 25.1$ $\left(\mathrm{B}\left(\mathrm{OC}\left(\mathrm{CH}_{3}\right)_{2}\right)_{2}\right), \quad 18.5 \quad\left(\mathrm{SiC}\left(\mathrm{CH}_{3}\right)_{3}\right), \quad-5.3$ $\left(\mathrm{Si}\left(\mathrm{CH}_{3}\right)_{2}\right)$. 
FTIR (thin film) $\mathrm{cm}^{-1}$ :

HRMS (ESI) $(m / z)$ :

$[\alpha]_{\mathrm{D}}^{25}$ : $2928(\mathrm{~m}), 2855(\mathrm{w}), 1682(\mathrm{~m}), 1613(\mathrm{~m}), 1437$ (s), $1369(\mathrm{~m}), 1252(\mathrm{~s}), 1158(\mathrm{~m}), 1124(\mathrm{~m}), 837$ (m), $777(\mathrm{w})$.

calc'd for $\mathrm{C}_{33} \mathrm{H}_{49} \mathrm{BN}_{2} \mathrm{NaO}_{5} \mathrm{Si}[\mathrm{M}+\mathrm{Na}]^{+}: 615.3$ 396.

found: 615.3397 .

$-16.9\left(c=0.23, \mathrm{CHCl}_{3}\right)$.

TLC (15\% ethyl acetate in hexanes), Rf: 0.30 (UV, CAM). 


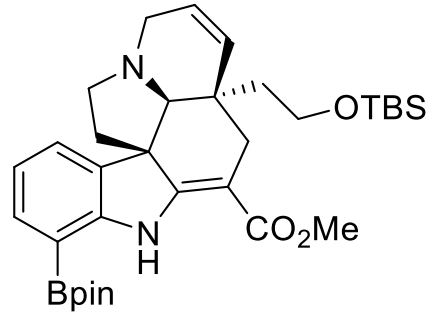

$(-)-12$
$\underset{\mathrm{MeOH}, 23^{\circ} \mathrm{C}, 48 \mathrm{~h}}{\stackrel{\text { diethylhydroxylamine }}{\longrightarrow}}$

$64 \%$

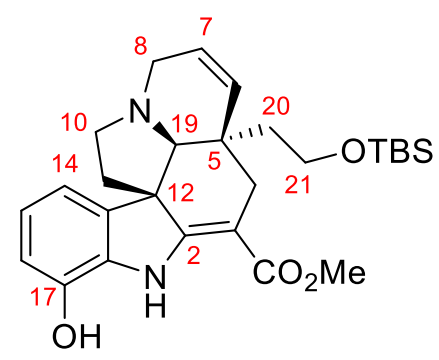

$(-)-16$

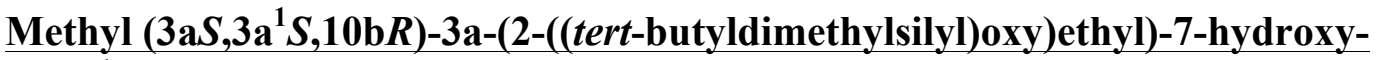
$3 \mathrm{a}^{3}, \mathrm{a}^{1}, 4,6,11,12$-hexahydro-1H-indolizino $[8,1-c d]$ carbazole-5-carboxylate; $\mathrm{C} 17$-alcohol $(-)-16$ :

$N, N$-Diethylhydroxylamine ( $22 \mu \mathrm{L}, 0.21 \mathrm{mmol} .20$ equiv) was added to a solution of boronic ester $(-)-12\left(6.3 \mathrm{mg}, 11 \mu \mathrm{mol}, 1\right.$ equiv) in methanol $(0.20 \mathrm{~mL})$ at $23{ }^{\circ} \mathrm{C}$. After $24 \mathrm{~h}$, Diethylhydroxylamine $(22 \mu \mathrm{L}, 0.21 \mathrm{mmol})$ was added to the reaction mixture additionally. After $24 \mathrm{~h}$, saturated aqueous ammonium chloride solution $(10 \mathrm{~mL})$ was added to the reaction mixture and diluted with dichloromethane $(10 \mathrm{~mL})$. The layers were separated, and the aqueous layer was extracted with dichloromethane $(2 \times 10 \mathrm{~mL})$. The combined organic layers were dried over anhydrous sodium sulfate, were filtered, and were concentrated under reduced pressure. The resulting residue was purified by flash column chromatography on silica gel (eluent: $5 \%$ ethyl acetate in hexanes to $15 \%$ ethyl acetate in hexanes) to afford $\mathrm{C} 17$ alcohol (-)-16 (3.2 mg, $7.0 \mu \mathrm{mol}, 64 \%)$ as a colorless oil. Structural assignments were made using additional information from gCOSY, gHSQC and gHMBC experiments.

${ }^{1} \mathrm{H}$ NMR $\left(600 \mathrm{MHz}, \mathrm{CD}_{3} \mathrm{CN}, 25^{\circ} \mathrm{C}\right)$ :

${ }^{13} \mathrm{C}$ NMR (150.9 MHz, $\left.\mathrm{CD}_{3} \mathrm{CN}, 25^{\circ} \mathrm{C}\right)$ :

FTIR (thin film) $\mathrm{cm}^{-1}$ : $\delta 8.83$ (br. s., $\left.1 \mathrm{H}, \mathrm{N}_{1} \mathbf{H}\right), 6.86(\mathrm{~d}, J=7.3 \mathrm{~Hz}, 1 \mathrm{H}$, $\left.\mathrm{C}_{14} \mathbf{H}\right), 6.77\left(\mathrm{t}, J=7.6 \mathrm{~Hz}, 1 \mathrm{H}, \mathrm{C}_{15} \mathbf{H}\right), 6.72(\mathrm{~d}, J$ $\left.=8.0 \mathrm{~Hz}, 1 \mathrm{H}, \mathrm{C}_{16} \mathbf{H}\right), 5.81-5.74\left(\mathrm{~m}, 2 \mathrm{H}, \mathrm{C}_{6} \mathbf{H}\right.$, $\left.\mathrm{C}_{7} \mathbf{H}\right), 3.73\left(\mathrm{~s}, 3 \mathrm{H}, \mathrm{CO}_{2} \mathrm{CH}_{3}\right), 3.50$ (ddd, $J=$ $\left.10.2,8.3,7.3 \mathrm{~Hz}, 1 \mathrm{H}, \mathrm{C}_{21} \mathbf{H}_{\mathrm{a}}\right), 3.43-3.36(\mathrm{~m}, 2 \mathrm{H}$, $\left.\mathrm{C}_{21} \mathbf{H}_{\mathrm{b}}, \mathrm{C}_{8} \mathbf{H}_{\mathrm{a}}\right), 3.14\left(\mathrm{~d}, J=16.0 \mathrm{~Hz}, 1 \mathrm{H}, \mathrm{C}_{8} \mathbf{H}_{\mathrm{b}}\right.$ ), $2.97\left(\mathrm{dd}, J=8.4,6.5 \mathrm{~Hz}, 1 \mathrm{H}, \mathrm{C}_{10} \mathbf{H}_{\mathrm{a}}\right), 2.68(\mathrm{~s}$, $\left.1 \mathrm{H}, \mathrm{C}_{19} \mathrm{H}\right), 2.66(\mathrm{ddd}, J=11.6,8.7,4.7 \mathrm{~Hz}, 1 \mathrm{H}$, $\left.\mathrm{C}_{10} \mathbf{H}_{\mathrm{b}}\right)$ 2.50-2.42 (m, 2H, $\left.\mathrm{C}_{4} \mathbf{H}_{2}\right), 1.98(\mathrm{td}, J=$ 11.5, $\left.6.4 \mathrm{~Hz}, 1 \mathrm{H}, \mathrm{C}_{11} \mathbf{H}_{\mathrm{a}}\right), 1.71(\mathrm{dd}, J=11.6,4.4$ $\left.\mathrm{Hz}, 1 \mathrm{H}, \mathrm{C}_{11} \mathbf{H}_{\mathrm{b}}\right), 1.11-1.07\left(\mathrm{~m}, 2 \mathrm{H}, \mathrm{C}_{20} \mathbf{H}_{2}\right), 0.77$ $\left(\mathrm{s}, 9 \mathrm{H}, \mathrm{SiC}\left(\mathrm{CH}_{3}\right)_{3}\right),-0.08$ to $-0.14(\mathrm{~m}, 6 \mathrm{H}$, $\left.\mathrm{Si}\left(\mathrm{CH}_{3}\right)_{2}\right)$.

$\delta 169.2\left(\mathrm{OC}(\mathrm{O}) \mathrm{CH}_{3}\right), 167.8\left(\mathbf{C}_{2}\right), 141.8\left(\mathbf{C}_{17}\right)$, $140.4\left(\mathbf{C}_{13}\right), 134.2\left(\mathbf{C}_{6}\right), 131.5\left(\mathbf{C}_{18}\right), 126.1\left(\mathbf{C}_{7}\right)$, $122.6\left(\mathbf{C}_{15}\right), 116.1\left(\mathbf{C}_{16}\right), 114.5\left(\mathbf{C}_{14}\right), 93.2\left(\mathbf{C}_{3}\right)$, $70.7\left(\mathbf{C}_{19}\right), 59.8\left(\mathbf{C}_{21}\right), 56.7\left(\mathbf{C}_{12}\right), 51.5\left(\mathbf{C}_{10}\right)$, $51.4\left(\mathbf{C}_{8}\right), 51.3\left(\mathrm{OC}(\mathrm{O}) \mathbf{C H}_{3}\right), 45.5\left(\mathbf{C}_{11}\right), 41.3$ $\left(\mathbf{C}_{5}\right), 37.9\left(\mathbf{C}_{20}\right), 29.9\left(\mathbf{C}_{4}\right), 26.2\left(\mathrm{SiC}\left(\mathbf{C H}_{3}\right)_{3}\right)$, $18.7\left(\mathrm{SiC}\left(\mathrm{CH}_{3}\right)_{3}\right),-5.3\left(\mathrm{Si}\left(\mathrm{CH}_{3}\right)_{2}\right)$.

3367 (br), 2925 (s), 2854 (m), 1613 (m), 1463 
(w), $1294(\mathrm{~m}), 1253(\mathrm{~m}), 1168(\mathrm{w}), 1091(\mathrm{w})$, $837(\mathrm{w}), 777(\mathrm{w})$.

HRMS (ESI) $(m / z)$ :

calc'd for $\mathrm{C}_{27} \mathrm{H}_{39} \mathrm{~N}_{2} \mathrm{O}_{4} \mathrm{Si}[\mathrm{M}+\mathrm{H}]^{+}$: 483.2674 .

found: 483.2675 .

$[\alpha]_{\mathrm{D}}^{25}:$

$-73.7\left(c=0.08, \mathrm{CHCl}_{3}\right)$.

TLC (20\% ethyl acetate in hexanes), Rf: 0.20 (UV, CAM). 


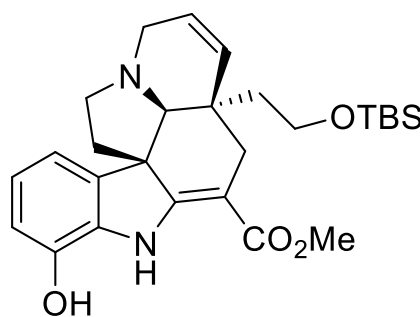

$(-)-16$

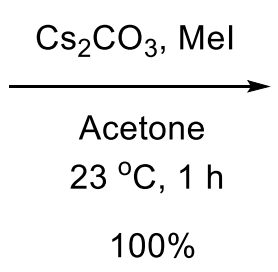

Methyl (3aS,3a1S,10bR)-3a-(2-((tert-butyldimethylsilyl)oxy)ethyl)-7-methoxy3a,3a1,4,6,11,12-hexahydro-1H-indolizino[8,1-cd]carbazole-5-carboxylate; C17-methyl ether $(-)-7$ :

Cesium carbonate (49 mg, $0.15 \mathrm{mmol}, 10$ equiv) and iodomethane $(9.3 \mu \mathrm{L}, 0.15 \mathrm{mmol}$, 10 equiv) were added to a solution of $\mathrm{C} 17$-alcohol (-)-16 (7.2 $\mathrm{mg}, 15 \mu \mathrm{mol}, 1$ equiv) in acetone $(0.30 \mathrm{~mL})$ at $23{ }^{\circ} \mathrm{C}$. After $1 \mathrm{~h}$, a solution of saturated aqueous ammonium chloride $(10 \mathrm{~mL})$ and dichloromethane $(10 \mathrm{~mL})$ were added and the layers were separated. The aqueous layer was extracted with dichloromethane $(2 \times 10 \mathrm{~mL})$ and the combined organic layers were dried with anhydrous sodium sulfate, were filtered, and were concentrated under reduced pressure. The resulting residue was purified by flash column chromatography on silica gel (eluent: 10\% ethyl acetate in hexanes) to afford C17-methyl ether (-)-7 (7.4 mg, $0.15 \mathrm{mmol}, 100 \%$ ) as a colorless oil. Structural assignments were made using additional information from gCOSY, gHSQC and gHMBC experiments.

${ }^{1} \mathrm{H}$ NMR $\left(600 \mathrm{MHz}, \mathrm{CDCl}_{3}, 25^{\circ} \mathrm{C}\right)$ :

$\delta 8.89$ (br. s., $\left.1 \mathrm{H}, \mathrm{N}_{1} \mathbf{H}\right), 6.86(\mathrm{~d}, J=7.3 \mathrm{~Hz}, 1 \mathrm{H}$, $\left.\mathrm{C}_{14} \mathbf{H}\right), 6.84\left(\mathrm{t}, J=7.3 \mathrm{~Hz}, 1 \mathrm{H}, \mathrm{C}_{15} \mathbf{H}\right), 6.75(\mathrm{~d}, J$ $\left.=7.3 \mathrm{~Hz}, 1 \mathrm{H}, \mathrm{C}_{16} \mathbf{H}\right), 5.81-5.73\left(\mathrm{~m}, 2 \mathrm{H}, \mathrm{C}_{6} \mathbf{H}\right.$, $\left.\mathrm{C}_{7} \mathbf{H}\right), 3.86\left(\mathrm{~s}, \quad 3 \mathrm{H}, \quad \mathrm{OCH}_{3}\right), 3.77(\mathrm{~s}, 3 \mathrm{H}$, $\left.\mathrm{CO}_{2} \mathrm{CH}_{3}\right), 3.53-3.39\left(\mathrm{~m}, 3 \mathrm{H}, \mathrm{C}_{21} \mathbf{H}_{2}, \mathrm{C}_{8} \mathbf{H}_{\mathrm{a}}\right), 3.17$ $\left(\mathrm{d}, J=15.6 \mathrm{~Hz}, 1 \mathrm{H}, \mathrm{C}_{8} \mathbf{H}_{\mathrm{b}}\right), 3.02(\mathrm{t}, J=7.3 \mathrm{~Hz}$, $\left.1 \mathrm{H}, \mathrm{C}_{10} \mathbf{H}_{\mathrm{a}}\right), 2.69\left(\mathrm{~s}, 1 \mathrm{H}, \mathrm{C}_{19} \mathbf{H}\right), 2.68$ (ddd, $J=$ 11.6, 8.7, $\left.4.7 \mathrm{~Hz}, 1 \mathrm{H}, \mathrm{C}_{10} \mathbf{H}_{\mathrm{b}}\right) 2.49\left(\mathrm{~s}, 2 \mathrm{H}, \mathrm{C}_{4} \mathbf{H}_{2}\right)$, $2.06\left(\mathrm{td}, J=11.4,6.4 \mathrm{~Hz}, 1 \mathrm{H}, \mathrm{C}_{11} \mathbf{H}_{\mathrm{a}}\right), 1.80(\mathrm{dd}$, $\left.J=11.6,4.4 \mathrm{~Hz}, 1 \mathrm{H}, \mathrm{C}_{11} \mathbf{H}_{\mathrm{b}}\right), 1.21-1.17$ (m, 2H, $\left.\mathrm{C}_{20} \mathbf{H}_{2}\right), 0.78$ (s, 9H, $\left.\mathrm{SiC}\left(\mathrm{CH}_{3}\right)_{3}\right),-0.10$ to -0.13 $\left(\mathrm{m}, 6 \mathrm{H}, \mathrm{Si}\left(\mathrm{CH}_{3}\right)_{2}\right)$.

${ }^{13} \mathrm{C}$ NMR $\left(150.9 \mathrm{MHz}, \mathrm{CDCl}_{3}, 25{ }^{\circ} \mathrm{C}\right)$ :

$\delta 168.7\left(\mathrm{OC}(\mathrm{O}) \mathrm{CH}_{3}\right), 166.8\left(\mathbf{C}_{2}\right), 144.4\left(\mathbf{C}_{17}\right)$, $138.9\left(\mathbf{C}_{13}\right), 133.9\left(\mathbf{C}_{6}\right), 132.2\left(\mathbf{C}_{18}\right), 124.9\left(\mathbf{C}_{7}\right)$, $121.4\left(\mathbf{C}_{15}\right), 114.1\left(\mathbf{C}_{14}\right), 110.4\left(\mathbf{C}_{16}\right), 92.5\left(\mathbf{C}_{3}\right)$, $70.0\left(\mathbf{C}_{19}\right), 59.5\left(\mathbf{C}_{21}\right), 56.1\left(\mathbf{C}_{12}\right), 55.7\left(\mathrm{OCH}_{3}\right)$ $51.1\left(2 \mathrm{C}, \mathbf{C}_{10}, \mathbf{C}_{8}\right), 50.8\left(\mathrm{OC}(\mathrm{O}) \mathbf{C H}_{3}\right), 44.7$ $\left(\mathbf{C}_{11}\right), 40.5\left(\mathbf{C}_{5}\right), 37.2\left(\mathbf{C}_{20}\right), 29.3\left(\mathbf{C}_{4}\right), 26.1$ $\left(\mathrm{SiC}\left(\mathrm{CH}_{3}\right)_{3}\right), 18.5\left(\mathrm{SiC}\left(\mathrm{CH}_{3}\right)_{3}\right),-5.4\left(\mathrm{Si}\left(\mathrm{CH}_{3}\right)_{2}\right)$.

FTIR (thin film) $\mathrm{cm}^{-1}$ :
2926 (s), 2854 (m), 1679 (m), 1614 (s), 1459(w), 1278 (s), 1254 (s), 1158 (m), 837 (w), 
775 (w).

HRMS (ESI) $(m / z)$ :

calc'd for $\mathrm{C}_{28} \mathrm{H}_{41} \mathrm{~N}_{2} \mathrm{O}_{4} \mathrm{Si}[\mathrm{M}+\mathrm{H}]^{+}$: 497.2830.

found: 497.2830.

$[\alpha]_{\mathrm{D}}^{25}$ :

$-105.5\left(c=0.08, \mathrm{CHCl}_{3}\right)$.

TLC (20\% ethyl acetate in hexanes), Rf: 0.30 (UV, CAM). 
<smiles>COC(=O)C1=C2Nc3c(OC)cccc3[C@@]23C=CCN2CC[C@](CCO[Sb])(C1)C23</smiles>

$(-)-7$

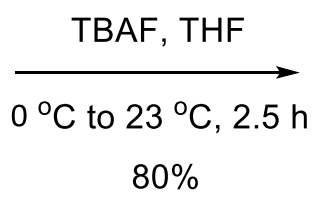

Methyl (3aS,3a $\left.\mathrm{a}^{1}, 10 \mathrm{~b} R\right)$-3a-(2-hydroxyethyl)-7-methoxy-3a,3a ${ }^{1}, 4,6,11,12$-hexahydro-1 $H$ indolizino $[8,1-c d]$ carbazole-5-carboxylate; C21-alcohol (-)-17:

Tetrabutylammonium fluoride solution (1.0 M in THF, $150 \mu \mathrm{L}, 0.15 \mathrm{mmol}, 10$ equiv) was added to a solution of C17-methyl ether (-)-7 (7.4 mg, $15 \mu \mathrm{mol}, 1$ equiv) in THF $(0.75$ $\mathrm{mL}$ ) at $0{ }^{\circ} \mathrm{C}$. After the addition, the reaction mixture was allowed to $23{ }^{\circ} \mathrm{C}$. After $2.5 \mathrm{~h}$, saturated aqueous sodium bicarbonate solution $(10 \mathrm{~mL})$ was added to the reaction mixture and diluted with ethyl acetate $(10 \mathrm{~mL})$. The layers were separated, and the aqueous layer was extracted with ethyl acetate $(2 \times 10 \mathrm{~mL})$. The combined organic layers were dried over anhydrous sodium sulfate, were filtered, and were concentrated under reduced pressure. The resulting residue was purified by flash column chromatography on silica gel (eluent: $30 \%$ ethyl acetate in hexanes to 50\% ethyl acetate in hexanes) to afford C21-alcohol (-)-17 (4.6 $\mathrm{mg}, 12 \mu \mathrm{mol}, 80 \%$ ) as a colorless oil. Structural assignments were made using additional information from gCOSY, gHSQC and gHMBC experiments.

${ }^{1} \mathrm{H} \mathrm{NMR}\left(600 \mathrm{MHz}, \mathrm{CDCl}_{3}, 25{ }^{\circ} \mathrm{C}\right)$ :

$\delta 8.87$ (br. s., $\left.1 \mathrm{H}, \mathrm{N}_{1} \mathbf{H}\right), 6.87(\mathrm{~d}, J=7.3 \mathrm{~Hz}, 1 \mathrm{H}$, $\left.\mathrm{C}_{14} \mathbf{H}\right), 6.84\left(\mathrm{t}, J=7.6 \mathrm{~Hz}, 1 \mathrm{H}, \mathrm{C}_{15} \mathbf{H}\right), 6.76(\mathrm{~d}, J$ $\left.=7.6 \mathrm{~Hz}, 1 \mathrm{H}, \mathrm{C}_{16} \mathbf{H}\right), 5.81(\mathrm{ddd}, J=9.8,4.7,1.1$ $\left.\mathrm{Hz}, 1 \mathrm{H}, \mathrm{C}_{7} \mathbf{H}\right) 5.76(\mathrm{dt}, J=9.8,1.8 \mathrm{~Hz}, 1 \mathrm{H}$, $\left.\mathrm{C}_{6} \mathbf{H}\right), 3.87\left(\mathrm{~s}, \quad 3 \mathrm{H}, \quad \mathrm{OCH}_{3}\right), 3.78(\mathrm{~s}, 3 \mathrm{H}$, $\mathrm{CO}_{2} \mathrm{CH}_{3}$ ), 3.56-3.43 (m, 3H, $\left.\mathrm{C}_{21} \mathbf{H}_{2}, \mathrm{C}_{8} \mathbf{H}_{\mathrm{a}}\right), 3.18$ $\left(\mathrm{d}, J=16 \mathrm{~Hz}, 1 \mathrm{H}, \mathrm{C}_{8} \mathbf{H}_{\mathrm{b}}\right), 3.03(\mathrm{t}, J=7.4 \mathrm{~Hz}$, $\left.1 \mathrm{H}, \mathrm{C}_{10} \mathbf{H}_{\mathrm{a}}\right), 2.74\left(\mathrm{~s}, 1 \mathrm{H}, \mathrm{C}_{19} \mathbf{H}\right), 2.69$ (ddd, $J=$ 11.2, 8.3, $\left.4.5 \mathrm{~Hz}, 1 \mathrm{H}, \mathrm{C}_{10} \mathbf{H}_{\mathrm{b}}\right) 2.55-2.47(\mathrm{~m}, 2 \mathrm{H}$, $\left.\mathrm{C}_{4} \mathbf{H}_{2}\right), 2.07\left(\mathrm{td}, J=11.4,6.4 \mathrm{~Hz}, 1 \mathrm{H}, \mathrm{C}_{11} \mathbf{H}_{\mathrm{a}}\right.$ ), $1.81\left(\mathrm{dd}, J=11.6,4.4 \mathrm{~Hz}, 1 \mathrm{H}, \mathrm{C}_{11} \mathbf{H}_{\mathrm{b}}\right), 1.21-$ 1.17 (m, 2H, $\mathrm{C}_{20} \mathbf{H}_{2}$ ).

${ }^{13} \mathrm{C} \mathrm{NMR}\left(150.9 \mathrm{MHz}, \mathrm{CDCl}_{3}, 25^{\circ} \mathrm{C}\right)$ :

$\delta 168.7\left(\mathrm{OC}(\mathrm{O}) \mathrm{CH}_{3}\right), 166.6\left(\mathbf{C}_{2}\right), 144.4\left(\mathbf{C}_{17}\right)$, $138.8\left(\mathbf{C}_{13}\right), 133.5\left(\mathbf{C}_{6}\right), 132.1\left(\mathbf{C}_{18}\right), 125.6\left(\mathbf{C}_{7}\right)$, $121.5\left(\mathbf{C}_{15}\right), 114.1\left(\mathbf{C}_{14}\right), 110.4\left(\mathbf{C}_{16}\right), 92.5\left(\mathbf{C}_{3}\right)$, $69.7\left(\mathbf{C}_{19}\right), 59.0\left(\mathbf{C}_{21}\right), 56.1\left(\mathbf{C}_{12}\right), 55.7\left(\mathrm{OCH}_{3}\right)$ $51.2\left(\mathbf{C}_{10}\right), 51.0\left(\mathrm{OC}(\mathrm{O}) \mathbf{C H}_{3}\right), 50.7\left(\mathbf{C}_{8}\right), 44.6$ $\left(\mathbf{C}_{11}\right), 40.5\left(\mathbf{C}_{5}\right), 37.4\left(\mathbf{C}_{20}\right), 29.7\left(\mathbf{C}_{4}\right)$.

FTIR (thin film) $\mathrm{cm}^{-1}$ : 3390 (br), 2923 (s), 2853 (m), 1677 (m), 1614 (m), $1459(\mathrm{w}), 1278(\mathrm{~m})$.

calc'd for $\mathrm{C}_{22} \mathrm{H}_{27} \mathrm{~N}_{2} \mathrm{O}_{4}[\mathrm{M}+\mathrm{H}]^{+}$: 383.1965 . 
found: 383.1967 .

$[\alpha]_{\mathrm{D}}^{25}:$

$-68.0\left(c=0.07, \mathrm{CHCl}_{3}\right)$.

TLC (50\% ethyl acetate in hexanes), Rf: 0.30 (UV, CAM). 


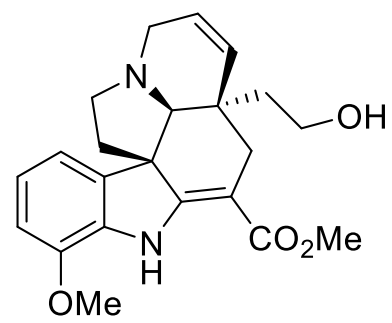

$(-)-17$

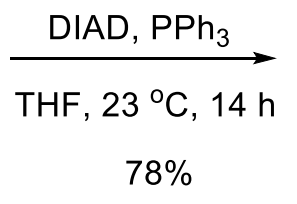

$78 \%$

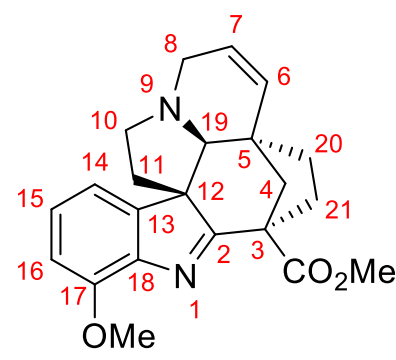

(+)-kopsifoline $E(3)$

(+)-Methyl (3aR,3a $\left.{ }^{1} S, 6 R, 11 \mathrm{~b} R\right)-8$-methoxy-4,5,12,13-tetrahydro-1H,3a ${ }^{1} H, 6 H-3 a, 6-$ methanoindolizino $\left[1^{\prime}, 8^{\prime}: 2,3,4\right]$ cyclohepta $[1,2-b]$ indole-6-carboxylate; $(+)$-kopsifoline $E$ (3):

Triphenylphosphine (30 mg, $0.11 \mathrm{mmol}, 7.0$ equiv) was added to a solution of C21alcohol (-)-17 (6.2 mg, $16 \mu \mathrm{mol}, 1$ equiv) in THF $(1.6 \mathrm{~mL})$ at $23{ }^{\circ} \mathrm{C}$ as a solid. Diisopropyl azodicarboxylate $\left(16 \mu \mathrm{L}, 81 \mu \mathrm{mol}, 5.0\right.$ equiv) was added to the reaction mixture at $23{ }^{\circ} \mathrm{C}$. After $14 \mathrm{~h}$, the reaction mixture was concentrated under reduced pressure. The resulting residue was purified by flash column chromatography on silica gel (eluent: $10 \%$ ethyl acetate in hexanes with $2.5 \%$ triethyl amine). The resulting residue was further purified by flash column chromatography on basic alumina (eluent: $20 \%$ ethyl acetate in hexanes) to afford (+)-kopsifoline E (3, $4.6 \mathrm{mg}, 13 \mu \mathrm{mol}, 78 \%)$ as a white solid. The optical rotation was acquired not only in chloroform ${ }^{7}$ (treated with $\mathrm{K}_{2} \mathrm{CO}_{3}$ ) for comparison with literature, but also in anhydrous dichloromethane ${ }^{1}$ prompted by incisive observations by Boger related to optical rotation of (-)-kopsifoline D (2). ${ }^{6}$ Structural assignments were made using additional information from $\mathrm{gCOSY}, \mathrm{gHSQC}$ and $\mathrm{gHMBC}$ experiments.

${ }^{1} \mathrm{H} \mathrm{NMR}\left(600 \mathrm{MHz}, \mathrm{CDCl}_{3}, 25^{\circ} \mathrm{C}\right)$ :

$\delta 7.16\left(\mathrm{t}, J=7.9,1 \mathrm{H}, \mathrm{C}_{15} \mathbf{H}\right), 7.08(\mathrm{~d}, J=7.3 \mathrm{~Hz}$, $\left.1 \mathrm{H}, \mathrm{C}_{14} \mathbf{H}\right), 6.85\left(\mathrm{~d}, J=8.2 \mathrm{~Hz}, 1 \mathrm{H}, \mathrm{C}_{16} \mathbf{H}\right), 5.75$ (ddd, $\left.J=10.0,4.2,1.3 \mathrm{~Hz}, 1 \mathrm{H}, \mathrm{C}_{7} \mathbf{H}\right), 5.63(\mathrm{dt}, J$ $\left.=10.3,1.3 \mathrm{~Hz}, 1 \mathrm{H}, \mathrm{C}_{6} \mathbf{H}\right), 3.97\left(\mathrm{~s}, 3 \mathrm{H}, \mathrm{OCH}_{3}\right)$, $3.75\left(\mathrm{~s}, 3 \mathrm{H}, \mathrm{CO}_{2} \mathrm{CH}_{3}\right), 3.56(\mathrm{dt}, J=17.6,2.0 \mathrm{~Hz}$, $\left.1 \mathrm{H}, \mathrm{C}_{8} \mathbf{H}_{\mathrm{a}}\right), 3.40$ (ddd, $J=17.6,4.1,1.5 \mathrm{~Hz}, 1 \mathrm{H}$, $\left.\mathrm{C}_{8} \mathbf{H}_{\mathrm{b}}\right), 3.33\left(\mathrm{td}, J=8.2,5.6 \mathrm{~Hz}, 1 \mathrm{H}, \mathrm{C}_{10} \mathbf{H}_{\mathrm{a}}\right.$ ), $3.28\left(\mathrm{td}, J=7.9,5.9 \mathrm{~Hz}, 1 \mathrm{H}, \mathrm{C}_{10} \mathbf{H}_{\mathrm{b}}\right), 2.75$ (br s, $\left.1 \mathrm{H}, \mathrm{C}_{19} \mathbf{H}\right), 2.63-2.56\left(\mathrm{~m}, 1 \mathrm{H}, \mathrm{C}_{11} \mathbf{H}_{\mathrm{a}}\right), 2.60$ (br d, $\left.J=12.4 \mathrm{~Hz}, 1 \mathrm{H}, \mathrm{C}_{4} \mathbf{H}_{\mathrm{a}}\right), 2.44(\mathrm{td}, J=12.0,8.7$ $\mathrm{Hz}, 1 \mathrm{H}, \mathrm{C}_{21} \mathbf{H}_{\mathrm{a}}$ ), 2.19 (ddt, $J=11.7,9.1,2.6 \mathrm{~Hz}$, $\left.1 \mathrm{H}, \mathrm{C}_{21} \mathbf{H}_{\mathrm{b}}\right), 2.07(\mathrm{dt}, J=13.1,6.5 \mathrm{~Hz}, 1 \mathrm{H}$, $\mathrm{C}_{11} \mathbf{H}_{\mathrm{b}}$ ), 1.76 (br d, $\left.J=12.4 \mathrm{~Hz}, 1 \mathrm{H}, \mathrm{C}_{4} \mathbf{H}_{\mathrm{b}}\right), 1.68$ (ddd, $\left.J=14.2,12.0,2.1 \mathrm{~Hz}, 1 \mathrm{H}, \mathrm{C}_{20} \mathbf{H}_{\mathrm{a}}\right), 1.20$ $1.12\left(\mathrm{~m}, 1 \mathrm{H}, \mathrm{C}_{20} \mathbf{H}_{\mathrm{b}}\right)$.

${ }^{13} \mathrm{C}$ NMR $\left(150.9 \mathrm{MHz}, \mathrm{CDCl}_{3}, 25^{\circ} \mathrm{C}\right)$ :

$\delta 186.8\left(\mathbf{C}_{2}\right), 172.8\left(\mathrm{OC}(\mathrm{O}) \mathrm{CH}_{3}\right), 151.1\left(\mathbf{C}_{18}\right)$, $149.5\left(\mathbf{C}_{13}\right), 142.5\left(\mathbf{C}_{17}\right), 132.7\left(\mathbf{C}_{6}\right), 127.4$ $\left(\mathbf{C}_{15}\right), \quad 125.5\left(\mathbf{C}_{7}\right), 114.1\left(\mathbf{C}_{14}\right), 110.4\left(\mathbf{C}_{16}\right)$, 
$69.4\left(\mathbf{C}_{19}\right), 63.4\left(\mathbf{C}_{12}\right), 56.7\left(\mathbf{C}_{3}\right), 55.9\left(\mathrm{OCH}_{3}\right)$, $52.6\left(\mathrm{OC}(\mathrm{O}) \mathbf{C H}_{3}\right), 51.1\left(\mathbf{C}_{10}\right), 47.1\left(\mathbf{C}_{8}\right), 44.3$ $\left(\mathbf{C}_{4}\right), 42.7\left(\mathbf{C}_{5}\right), 39.2\left(\mathbf{C}_{21}\right), 35.1\left(\mathbf{C}_{20}\right), 34.9$ $\left(\mathbf{C}_{11}\right)$.

FTIR (thin film) $\mathrm{cm}^{-1}$ : 2922 (s), 2852 (m), 1738 (s), 1485 (m), 1327 (s), 749 (w).

HRMS (ESI) $(m / z)$ :

calc'd for $\mathrm{C}_{22} \mathrm{H}_{24} \mathrm{~N}_{2} \mathrm{NaO}_{3}[\mathrm{M}+\mathrm{Na}]^{+}$: 387.1679. found: 387.1682 .

$[\alpha]_{\mathrm{D}}^{25}:$

$+44.3\left(c=0.07, \mathrm{CHCl}_{3}\right) ;+65.1(c=0.07$, $\mathrm{CH}_{2} \mathrm{Cl}_{2}$ ).

TLC (50\% ethyl acetate in hexanes), Rf: 0.40 (UV, CAM). 
Table S3. Comparison of ${ }^{1} \mathrm{H}$ NMR data of (+)-kopsifoline E with literature data

\begin{tabular}{|l|l|l|}
\hline Assignment & $\begin{array}{l}\text { Naturally occurring (+)-kopsifoline E (400 } \\
\left.\text { MHz, } \mathrm{CDCl}_{3}\right)^{8}\end{array}$ & $\begin{array}{l}\text { Movassaghi's synthetic (+)-kopsifoline E (600 } \\
\left.\text { MHz, } \mathrm{CDCl}_{3}\right)\end{array}$ \\
\hline $\mathrm{N} 1-\mathrm{H}$ & - & - \\
\hline $\mathrm{C} 2$ & - & - \\
\hline $\mathrm{C} 3$ & - & - \\
\hline $\mathrm{C} 4$ & $1.76(\mathrm{dd}, J=12,2 \mathrm{~Hz}, 1 \mathrm{H})$ & $1.76(\mathrm{br} \mathrm{d}, J=12.4 \mathrm{~Hz}, 1 \mathrm{H})$ \\
& $2.60(\mathrm{br}, J=12 \mathrm{~Hz}, 1 \mathrm{H})$ & $2.60(\mathrm{br} \mathrm{d}, J=12.4 \mathrm{~Hz}, 1 \mathrm{H})$ \\
\hline $\mathrm{C} 5$ & - & - \\
\hline $\mathrm{C} 6$ & $5.64(\mathrm{dt}, J=10,2 \mathrm{~Hz}, 1 \mathrm{H})$ & $5.63(\mathrm{dt}, J=10.3,1.3 \mathrm{~Hz}, 1 \mathrm{H})$ \\
\hline $\mathrm{C} 7$ & $5.77(\mathrm{ddd}, J=10,4,2 \mathrm{~Hz}, 1 \mathrm{H})$ & $5.75(\mathrm{ddd}, J=10.0,4.2,1.3 \mathrm{~Hz}, 1 \mathrm{H})$ \\
\hline $\mathrm{C} 8$ & $3.41(\mathrm{ddd}, J=17,4,2 \mathrm{~Hz}, 1 \mathrm{H})$, & $3.40(\mathrm{ddd}, J=17.6,4.1,1.5 \mathrm{~Hz}, 1 \mathrm{H})$ \\
& $3.58(\mathrm{br}, \mathrm{d}, J=17 \mathrm{~Hz}, 1 \mathrm{H})$ & $3.56(\mathrm{dt}, J=17.6,2.0 \mathrm{~Hz}, 1 \mathrm{H})$ \\
\hline $\mathrm{C} 10$ & $3.32(\mathrm{~m}, 1 \mathrm{H})$ & $3.28(\mathrm{td}, J=7.9,5.9 \mathrm{~Hz}, 1 \mathrm{H})$ \\
& $3.34(\mathrm{td}, J=8,6 \mathrm{~Hz}, 1 \mathrm{H})$ & $3.33(\mathrm{td}, J=8.2,5.6 \mathrm{~Hz}, 1 \mathrm{H})$ \\
\hline $\mathrm{C} 11$ & $2.08(\mathrm{~m}, 1 \mathrm{H})$ & $2.07(\mathrm{dt}, J=13.1,6.5 \mathrm{~Hz}, 1 \mathrm{H})$ \\
& $2.59(\mathrm{~m}, 1 \mathrm{H})$ & $2.63-2.56(\mathrm{~m}, 1 \mathrm{H})$ \\
\hline $\mathrm{C} 12$ & - & - \\
\hline $\mathrm{C} 13$ & - & - \\
\hline $\mathrm{C} 14$ & $7.10(\mathrm{br}, \mathrm{d}, J=8 \mathrm{~Hz}, 1 \mathrm{H})$ & $7.08(\mathrm{~d}, J=7.3 \mathrm{~Hz}, 1 \mathrm{H})$ \\
\hline $\mathrm{C} 15$ & $7.17(\mathrm{t}, J=8 \mathrm{~Hz}, 1 \mathrm{H})$ & $7.16(\mathrm{t}, J=7.9 \mathrm{~Hz}, 1 \mathrm{H})$ \\
\hline $\mathrm{C} 16$ & $6.86(\mathrm{dd}, J=8,2 \mathrm{~Hz}, 1 \mathrm{H})$ & $6.85(\mathrm{~d}, J=8.2 \mathrm{~Hz}, 1 \mathrm{H})$ \\
\hline $\mathrm{C} 17$ & - & - \\
\hline $17-\mathrm{OCH}$ & & $3.97(\mathrm{~s}, 3 \mathrm{H})$ \\
\hline $\mathrm{C} 18$ & $3.97(\mathrm{~s}, 3 \mathrm{H})$ & - \\
\hline $\mathrm{C} 19$ & - & $2.75(\mathrm{br} \mathrm{s}, 1 \mathrm{H})$ \\
\hline $\mathrm{C} 20$ & $2.76(\mathrm{br} \mathrm{s}, 1 \mathrm{H})$ & $1.20-1.12(\mathrm{~m}, 1 \mathrm{H})$ \\
& $1.20(\mathrm{~m}, 1 \mathrm{H})$ & $1.68(\mathrm{ddd}, J=14.2,12,2.1 \mathrm{~Hz}, 1 \mathrm{H})$ \\
\hline $\mathrm{C} 21$ & $1.69(\mathrm{~m}, 1 \mathrm{H})$ & $2.19(\mathrm{ddt}, J=11.7,9.1,2.6 \mathrm{~Hz}, 1 \mathrm{H})$ \\
& $2.19(\mathrm{ddt}, J=12,9,2 \mathrm{~Hz}, 1 \mathrm{H})$ & $2.44(\mathrm{td}, J=12.0,8.7 \mathrm{~Hz}, 1 \mathrm{H})$ \\
\hline $\mathrm{CO} \mathrm{CH}_{3}$ & $2.44(\mathrm{td}, J=12,8 \mathrm{~Hz}, 1 \mathrm{H})$ & $3.75(\mathrm{~s}, 3 \mathrm{H})$ \\
\hline & $3.79(\mathrm{~s}, 3 \mathrm{H})$ & \\
\hline & & \\
\hline
\end{tabular}


Table S4. Comparison of ${ }^{13} \mathrm{C}$ NMR data of (+)-kopsifoline E with literature data

\begin{tabular}{|l|c|c|c|}
\hline Assignment & $\begin{array}{l}\text { Naturally occurring (+)-kopsifoline E }(100 \mathrm{MHz}, \\
\left.\mathrm{CDCl}_{3}\right)^{8}{ }^{8} \text { Chemical Shift Difference }{ }^{10} \\
\Delta=\delta \text { (Movassaghi's synthetic, solvent ref. } 877.16)-\delta \text { (naturally occurring) }\end{array}$ & $\begin{array}{l}\text { Movassaghi's synthetic (+)-kopsifoline } \\
\left.\text { E } 150 \mathrm{MHz}, \mathrm{CDCl}_{3}\right)\end{array}$ \\
\hline $\mathrm{C} 2$ & 186.7 & 0.1 & 186.8 \\
\hline $\mathrm{C} 3$ & 56.5 & 0.2 & 56.7 \\
\hline $\mathrm{C} 4$ & 44.1 & 0.2 & 44.3 \\
\hline $\mathrm{C} 5$ & 42.4 & 0.3 & 42.7 \\
\hline $\mathrm{C} 6$ & 132.5 & 0.2 & 132.7 \\
\hline $\mathrm{C} 7$ & 125.1 & 0.4 & 125.5 \\
\hline $\mathrm{C} 8$ & 46.9 & 0.2 & 47.1 \\
\hline $\mathrm{C} 10$ & 50.9 & 0.2 & 51.1 \\
\hline $\mathrm{C} 11$ & 34.7 & 0.2 & 34.9 \\
\hline $\mathrm{C} 12$ & 63.2 & 0.2 & 63.4 \\
\hline $\mathrm{C} 13$ & 149.2 & 0.3 & 149.5 \\
\hline $\mathrm{C} 14$ & 113.9 & 0.2 & 114.1 \\
\hline $\mathrm{C} 15$ & 127.2 & 0.2 & 127.4 \\
\hline $\mathrm{C} 16$ & 110.2 & 0.2 & 110.4 \\
\hline $\mathrm{C} 17$ & 142.4 & 0.1 & 142.5 \\
\hline $17-\mathrm{OCH}_{3}$ & 55.7 & 0.2 & 55.9 \\
\hline $\mathrm{C} 18$ & 150.9 & 0.2 & 151.1 \\
\hline $\mathrm{C} 19$ & 69.2 & 0.2 & 69.4 \\
\hline $\mathrm{C} 20$ & 35.0 & 0.1 & 35.1 \\
\hline $\mathrm{C}_{21}$ & 39.0 & 0.2 & 39.2 \\
\hline $\mathrm{CO}_{2} \mathrm{CH}_{3}$ & 52.4 & 0.2 & 52.6 \\
\hline $\mathrm{CO} \mathrm{CH}_{3}$ & 172.5 & 0.3 & 172.8 \\
\hline
\end{tabular}




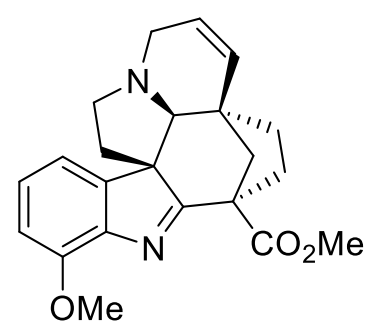

(+)-kopsifoline E (3)

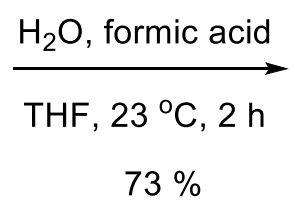

$73 \%$

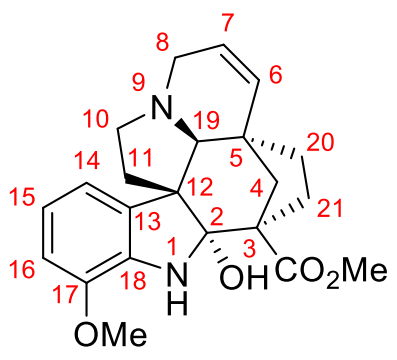

(-)-kopsifoline A (1)

(-)-Methyl (3a $\left.R, 3 a^{1} S, 6 R, 6 a R, 11 b R\right)-6 a-h y d r o x y-8-m e t h o x y-4,5,6 a, 7,12,13-h e x a h y d r o-$ $\underline{1 H, 3 a^{1} H, 6 H-3 a, 6-m e t h a n o i n d o l i z i n o ~}\left[1^{\prime}, 8^{\prime}: 2,3,4\right]$ cyclohepta $[1,2-b]$ indole-6-carboxylate; (-)-kopsifoline A (1):

Water $(0.12 \mathrm{~mL})$ and Formic acid $(50 \mu \mathrm{L})$ were added to a solution of $(+)$-kopsifoline $\mathrm{E}$ (3, $2.4 \mathrm{mg}, 6.6 \mu \mathrm{mol}, 1$ equiv) in THF at $23{ }^{\circ} \mathrm{C}$. After $2 \mathrm{~h}$, the reaction mixture was concentrated under reduced pressure. The resulting residue was purified by flash column chromatography on silica gel (eluent: $50 \%$ ethyl acetate in hexanes to $100 \%$ ethyl acetate) to afforded (-)-kopsifoline A (1, $2.0 \mathrm{mg}, 4.8 \mu \mathrm{mol}, 73 \%)$ as a white solid. Structural assignments were made using additional information from gCOSY, gHSQC and gHMBC experiments.

${ }^{1} \mathrm{H}$ NMR $\left(600 \mathrm{MHz}, \mathrm{CDCl}_{3}, 25^{\circ} \mathrm{C}\right)$ :

$\delta 6.76\left(\mathrm{~d}, J=7.6 \mathrm{~Hz}, 1 \mathrm{H}, \mathrm{C}_{14} \mathbf{H}\right), 6.70(\mathrm{t}, J=7.8$, $\left.1 \mathrm{H}, \mathrm{C}_{15} \mathbf{H}\right), 6.65\left(\mathrm{~d}, J=8.0 \mathrm{~Hz}, 1 \mathrm{H}, \mathrm{C}_{16} \mathbf{H}\right), 5.99$ (br s, $\left.1 \mathrm{H}, \mathrm{N}_{1} \mathbf{H}\right) 5.73$ (ddd, $J=9.4,4.7,1.5 \mathrm{~Hz}$, $\left.1 \mathrm{H}, \mathrm{C}_{7} \mathbf{H}\right), 5.48\left(\mathrm{dt}, J=9.4,1.8 \mathrm{~Hz}, 1 \mathrm{H}, \mathrm{C}_{6} \mathbf{H}\right)$, $3.82\left(\mathrm{~s}, 3 \mathrm{H}, \mathrm{OCH}_{3}\right), 3.76\left(\mathrm{~s}, 3 \mathrm{H}, \mathrm{CO}_{2} \mathrm{CH}_{3}\right), 3.54$ (dd, $\left.J=16.2,4.2 \mathrm{~Hz}, 1 \mathrm{H}, \mathrm{C}_{8} \mathbf{H}_{\mathrm{a}}\right), 3.25(\mathrm{t}, J=8.0$ $\left.\mathrm{Hz}, 1 \mathrm{H}, \mathrm{C}_{10} \mathbf{H}_{\mathrm{a}}\right), 3.06(\mathrm{td}, J=11.9,7.8 \mathrm{~Hz}, 1 \mathrm{H}$, $\mathrm{C}_{11} \mathbf{H}_{\mathrm{a}}$ ), 2.84 (dt, $J=16.3,2.2 \mathrm{~Hz}, 1 \mathrm{H}, \mathrm{C}_{8} \mathbf{H}_{\mathrm{b}}$ ), 2.77 (br s, 1H, OH), 2.48 (br s, $1 \mathrm{H}, \mathrm{C}_{19} \mathbf{H}$ ), 2.39 (ddd, $\left.J=11.3,8.4,6.9 \mathrm{~Hz}, 1 \mathrm{H}, \mathrm{C}_{10} \mathbf{H}_{\mathrm{b}}\right), 2.23$ (d, $J=12.4 \mathrm{~Hz}, 1 \mathrm{H}, \mathrm{C}_{4} \mathbf{H}_{\mathrm{a}}$ ), 1.88 (br d, $J=12.4 \mathrm{~Hz}$, $\left.1 \mathrm{H}, \mathrm{C}_{4} \mathbf{H}_{\mathrm{b}}\right), 1.74-1.70\left(\mathrm{~m}, 2 \mathrm{H}, \mathrm{C}_{21} \mathbf{H}_{2}\right), 1.58-1.51$ $\left(\mathrm{m}, 1 \mathrm{H}, \mathrm{C}_{20} \mathbf{H}_{\mathrm{a}}\right), 1.42(\mathrm{dd}, J=12.5,6.4 \mathrm{~Hz}, 1 \mathrm{H}$, $\left.\mathrm{C}_{11} \mathbf{H}_{\mathrm{b}}\right), 1.32-1.20\left(\mathrm{~m}, 1 \mathrm{H}, \mathrm{C}_{20} \mathbf{H}_{\mathrm{b}}\right)$.

${ }^{13} \mathrm{C} \mathrm{NMR}\left(150.9 \mathrm{MHz}, \mathrm{CDCl}_{3}, 25{ }^{\circ} \mathrm{C}\right)$ :

$\delta 176.5\left(\mathrm{OC}(\mathrm{O}) \mathrm{CH}_{3}\right), 143.2\left(\mathbf{C}_{17}\right), 138.9\left(\mathbf{C}_{13}\right)$, $136.1\left(\mathbf{C}_{18}\right), 133.0\left(\mathbf{C}_{6}\right), 125.7\left(\mathbf{C}_{7}\right), 119.7\left(\mathbf{C}_{15}\right)$, $115.0\left(\mathbf{C}_{14}\right), 109.7\left(\mathbf{C}_{16}\right), 97.3\left(\mathbf{C}_{2}\right), 75.8\left(\mathbf{C}_{19}\right)$, $60.2\left(\mathbf{C}_{3}\right), 56.5\left(\mathbf{C}_{12}\right), 55.6\left(\mathrm{OCH}_{3}\right), 54.1\left(\mathbf{C}_{10}\right)$, $53.3\left(\mathbf{C}_{8}\right), 52.3\left(\mathrm{OC}(\mathrm{O}) \mathbf{C H}_{3}\right), 45.1\left(\mathbf{C}_{5}\right), 38.4$ $\left(\mathbf{C}_{4}\right), 35.3\left(\mathbf{C}_{20}\right), 35.1\left(\mathbf{C}_{11}\right), 29.8\left(\mathbf{C}_{21}\right)$.

FTIR (thin film) $\mathrm{cm}^{-1}$ : 3365 (br), 2923 (s), 2850 (m), 1713 (m), 1495 (m), $1232(\mathrm{~s}), 732(\mathrm{w})$.

HRMS (ESI) $(m / z)$ :

calc'd for $\mathrm{C}_{22} \mathrm{H}_{27} \mathrm{~N}_{2} \mathrm{O}_{4}[\mathrm{M}+\mathrm{H}]^{+}$: 383.1965 .

found: 383.1963 . 
$[\alpha]_{\mathrm{D}}^{25}: \quad-11.7\left(c=0.10 \mathrm{CHCl}_{3}\right)$.

TLC (50\% ethyl acetate in hexanes), Rf: 0.20 (UV, CAM). 
Table S5. Comparison of ${ }^{1} \mathrm{H}$ NMR data of (-)-kopsifoline A with literature data

\begin{tabular}{|c|c|c|}
\hline Assignment & 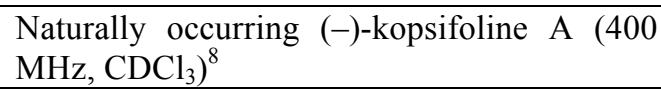 & 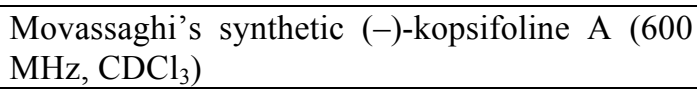 \\
\hline N1-H & 5.98 (br. s) & 5.99 (br. s, $1 \mathrm{H}$ ) \\
\hline $\mathrm{C} 2$ & - & - \\
\hline $2-\mathrm{OH}$ & 2.99 (br. s) & 2.77 (br. s, $1 \mathrm{H})$ \\
\hline $\mathrm{C} 3$ & - & - \\
\hline $\mathrm{C} 4$ & $\begin{array}{l}1.86(\mathrm{dd}, J=13,1 \mathrm{~Hz}, 1 \mathrm{H}) \\
2.23(\mathrm{dd}, J=13,1 \mathrm{~Hz}, 1 \mathrm{H})\end{array}$ & $\begin{array}{l}1.88(\mathrm{~d}, J=12.4 \mathrm{~Hz}, 1 \mathrm{H}) \\
2.23(\mathrm{~d}, J=12.4 \mathrm{~Hz})\end{array}$ \\
\hline $\mathrm{C} 5$ & - & - \\
\hline C6 & $5.47(\mathrm{dt}, J=10,2 \mathrm{~Hz}, 1 \mathrm{H})$ & $5.48(\mathrm{dt}, J=9.4,1.8 \mathrm{~Hz}, 1 \mathrm{H})$ \\
\hline $\mathrm{C} 7$ & $5.72(\mathrm{ddd}, J=10,5,2 \mathrm{~Hz}, 1 \mathrm{H})$ & $5.73(\mathrm{ddd}, J=9.4,4.7,1.5 \mathrm{~Hz}, 1 \mathrm{H})$ \\
\hline $\mathrm{C} 8$ & $\begin{array}{l}2.83(\mathrm{dt}, J=16,2 \mathrm{~Hz}, 1 \mathrm{H}) \\
3.53(\mathrm{ddd}, J=16,5,2 \mathrm{~Hz}, 1 \mathrm{H})\end{array}$ & $\begin{array}{l}2.84(\mathrm{dt}, J=16.3,2.2 \mathrm{~Hz}, 1 \mathrm{H}) \\
3.54(\mathrm{dd}, J=16.2,4.2 \mathrm{~Hz}, 1 \mathrm{H})\end{array}$ \\
\hline $\mathrm{C} 10$ & $\begin{array}{l}2.38(\mathrm{ddd}, J=12,8,7 \mathrm{~Hz}, 1 \mathrm{H}) \\
3.24(\mathrm{t}, J=8 \mathrm{~Hz}, 1 \mathrm{H})\end{array}$ & $\begin{array}{l}2.39(\mathrm{ddd}, J=11.3,8.4,6.9 \mathrm{~Hz}, 1 \mathrm{H}) \\
3.25(\mathrm{t}, J=8.0 \mathrm{~Hz}, 1 \mathrm{H})\end{array}$ \\
\hline C11 & $\begin{array}{l}1.40(\mathrm{dd}, J=13,7 \mathrm{~Hz}, 1 \mathrm{H}) \\
3.05(\mathrm{ddd}, J=13,12,8 \mathrm{~Hz}, 1 \mathrm{H})\end{array}$ & $\begin{array}{l}1.42(\mathrm{dd}, J=12.5,6.4 \mathrm{~Hz}, 1 \mathrm{H}) \\
3.06(\mathrm{td}, J=11.9,7.8 \mathrm{~Hz}, 1 \mathrm{H})\end{array}$ \\
\hline $\mathrm{C} 12$ & - & - \\
\hline $\mathrm{C} 13$ & - & - \\
\hline $\mathrm{C} 14$ & $6.75(\mathrm{dd}, J=8,1 \mathrm{~Hz}, 1 \mathrm{H})$ & $6.76(\mathrm{~d}, J=7.6 \mathrm{~Hz}, 1 \mathrm{H})$ \\
\hline $\mathrm{C} 15$ & $6.69(\mathrm{t}, J=8 \mathrm{~Hz}, 1 \mathrm{H})$ & $6.70(\mathrm{t}, J=7.8 \mathrm{~Hz}, 1 \mathrm{H})$ \\
\hline C16 & $6.65(\mathrm{dd}, J=8,1 \mathrm{~Hz}, 1 \mathrm{H})$ & $6.65(\mathrm{~d}, J=8.0 \mathrm{~Hz}, 1 \mathrm{H})$ \\
\hline $\mathrm{C} 17$ & - & - \\
\hline $17-\mathrm{OCH}_{3}$ & $3.82(\mathrm{~s}, 3 \mathrm{H})$ & $3.82(\mathrm{~s}, 3 \mathrm{H})$ \\
\hline $\mathrm{C} 18$ & - & - \\
\hline C19 & 2.48 (br. s, $1 \mathrm{H}$ ) & 2.48 (br. s, $1 \mathrm{H}$ ) \\
\hline $\mathrm{C} 20$ & $\begin{array}{l}1.26(\mathrm{~m}, 1 \mathrm{H}) \\
1.52(\mathrm{~m}, 1 \mathrm{H})\end{array}$ & $\begin{array}{l}1.32-1.20(\mathrm{~m}, 1 \mathrm{H}) \\
1.58-1.51(\mathrm{~m}, 1 \mathrm{H})\end{array}$ \\
\hline $\mathrm{C} 21$ & $\begin{array}{l}1.71(\mathrm{~m}, 1 \mathrm{H}) \\
1.71(\mathrm{~m}, 1 \mathrm{H})\end{array}$ & $1.74-1.70(\mathrm{~m}, 2 \mathrm{H})$ \\
\hline $\mathrm{CO}_{2} \mathrm{CH}_{3}$ & $3.75(\mathrm{~s}, 3 \mathrm{H})$ & $3.76(\mathrm{~s}, 3 \mathrm{H})$ \\
\hline
\end{tabular}


Table S6. Comparison of ${ }^{13} \mathrm{C}$ NMR data of (-)-kopsifoline A with literature data

\begin{tabular}{|l|c|c|c|}
\hline Assignment & $\begin{array}{l}\text { Naturally occurring (-)-kopsifoline A }(100 \mathrm{MHz}, \\
\left.\mathrm{CDCl}_{3}\right)^{8}{ }^{8} \text { Chemical Shift Difference }{ }^{10} \\
\Delta=\delta \text { (Movassaghi's synthetic, solvent ref. } 877.16)-\delta \text { (naturally occurring) }\end{array}$ & $\begin{array}{l}\text { Movassaghi's synthetic (-)-kopsifoline } \\
\mathrm{A}\left(150 \mathrm{MHz}, \mathrm{CDCl}_{3}\right)\end{array}$ \\
\hline $\mathrm{C} 2$ & 97.1 & 0.2 & 97.3 \\
\hline $\mathrm{C} 3$ & 60.0 & 0.2 & 60.2 \\
\hline $\mathrm{C} 4$ & 38.1 & 0.3 & 38.4 \\
\hline $\mathrm{C} 5$ & 44.8 & 0.3 & 45.1 \\
\hline $\mathrm{C} 6$ & 132.8 & 0.2 & 133.0 \\
\hline $\mathrm{C} 7$ & 125.4 & 0.3 & 125.7 \\
\hline $\mathrm{C} 8$ & 53.0 & 0.3 & 53.3 \\
\hline $\mathrm{C} 10$ & 53.9 & 0.2 & 54.1 \\
\hline $\mathrm{C} 11$ & 34.8 & 0.3 & 35.1 \\
\hline $\mathrm{C} 12$ & 56.2 & 0.3 & 56.5 \\
\hline $\mathrm{C} 13$ & 138.6 & 0.3 & 138.9 \\
\hline $\mathrm{C} 14$ & 114.7 & 0.3 & 115.0 \\
\hline $\mathrm{C} 15$ & 119.4 & 0.3 & 119.7 \\
\hline $\mathrm{C} 16$ & 109.4 & 0.3 & 109.7 \\
\hline $\mathrm{C} 17$ & 143.0 & 0.2 & 143.2 \\
\hline $17-\mathrm{OCH}_{3}$ & 55.3 & 0.3 & 55.6 \\
\hline $\mathrm{C} 18$ & 135.9 & 0.2 & 136.1 \\
\hline $\mathrm{C} 19$ & 75.6 & 0.2 & 75.8 \\
\hline $\mathrm{C} 20$ & 35.1 & 0.2 & 35.3 \\
\hline $\mathrm{C}_{21}$ & 29.5 & 0.3 & 29.8 \\
\hline $\mathrm{CO}_{2} \mathrm{CH}_{3}$ & 52.0 & 0.3 & 52.3 \\
\hline $\mathrm{CO} \mathrm{CH}_{3}$ & 176.3 & 0.3 & 176.5 \\
\hline
\end{tabular}


Computational Information. All computational studies were conducted using the Gaussian09 software package. ${ }^{13}$ The geometries (gas phase) in the ground state of $(+)-\mathbf{1 1}$ and (-)-8 were optimized with Merck Molecular Force Field (MMFF) ${ }^{14}$ followed by density functional theory at B3LYP level with $6-311+\mathrm{g}(\mathrm{d}, \mathrm{p})$ as basis set (Gaussian09, by Gaussian, Inc.). ${ }^{13}$ The electrostatic potential (ESP) derived charge was calculated using the lowest energy conformation.<smiles>CCOCCC1CCCN2CC=CCC12CCc1cc2ccccc2[nH]1</smiles>

$(+)-11$

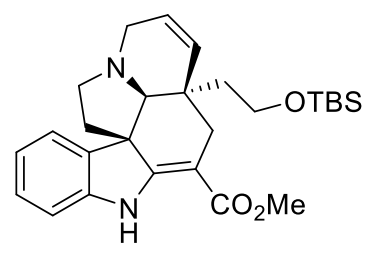

$(-)-8$
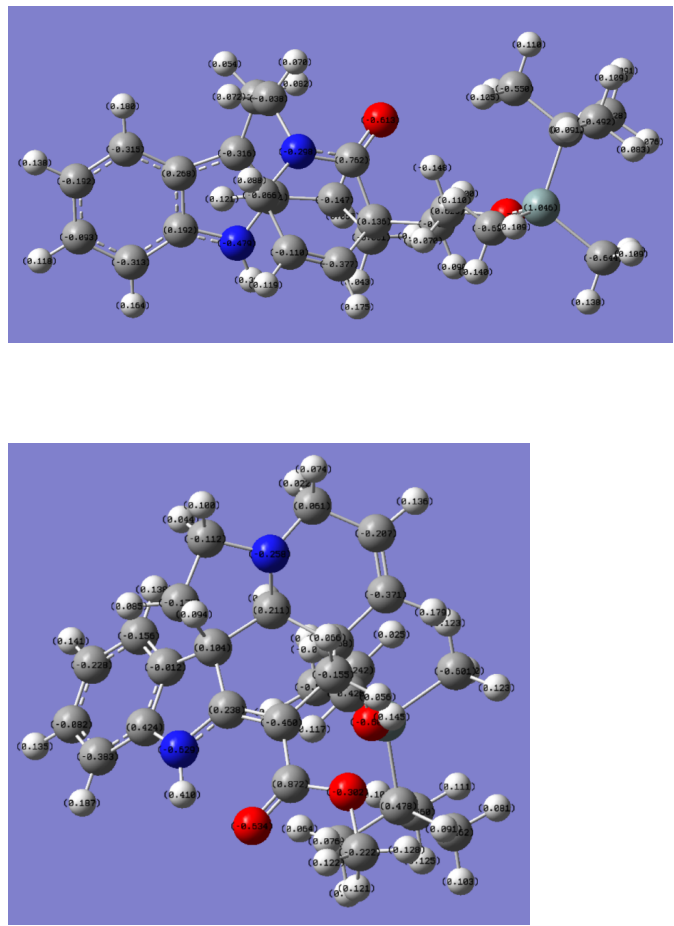

Figure S1. Comparison of atomic charges between vinylogous urethane $\mathbf{8}$ and indole $\mathbf{1 1 .}$

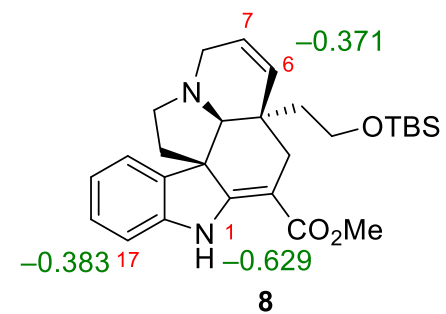

8

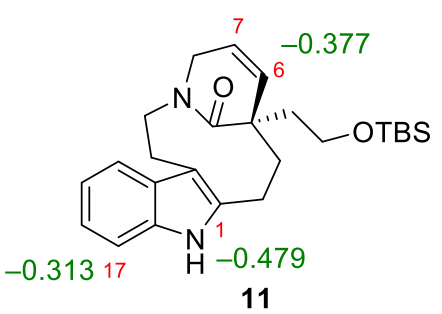

11 
Table S7. Optimized Cartesian Coordinates and Zero Point Vibrational Energy Corrected Absolute Energy in Hartree of indole $(+)-11$ : $E=-1524.21882657$ a.u.

\begin{tabular}{|c|c|c|c|c|c|c|c|}
\hline $\mathrm{C}$ & 6.94259 & 0.90416 & -1.28511 & $\mathrm{H}$ & 5.62026 & 2.50164 & -0.71952 \\
\hline $\mathrm{C}$ & 7.2285 & -0.47215 & -1.21218 & $\mathrm{H}$ & 4.26131 & -2.37531 & 1.11554 \\
\hline $\mathrm{C}$ & 6.40074 & -1.34106 & -0.51142 & $\mathrm{H}$ & 2.62779 & -1.46223 & 2.99692 \\
\hline $\mathrm{C}$ & 5.27716 & -0.79974 & 0.11552 & $\mathrm{H}$ & 1.83292 & 0.05648 & 2.657 \\
\hline $\mathrm{C}$ & 4.96854 & 0.58548 & 0.05867 & $\mathrm{H}$ & 1.41048 & -2.59114 & 1.1768 \\
\hline $\mathrm{C}$ & 5.82427 & 1.4378 & -0.65843 & $\mathrm{H}$ & 0.39048 & -1.89205 & 2.41763 \\
\hline $\mathrm{N}$ & 4.28905 & -1.40064 & 0.86573 & $\mathrm{H}$ & 1.23765 & 3.08046 & 0.16711 \\
\hline $\mathrm{C}$ & 3.37072 & -0.44897 & 1.29321 & $\mathrm{H}$ & 2.43011 & 2.66573 & -1.07035 \\
\hline $\mathrm{C}$ & 3.75717 & 0.78793 & 0.81574 & $\mathrm{H}$ & 3.69304 & 2.92706 & 0.97134 \\
\hline $\mathrm{C}$ & 2.22814 & -0.84242 & 2.18374 & $\mathrm{H}$ & 2.47816 & 2.09629 & 1.92305 \\
\hline $\mathrm{C}$ & 1.04203 & -1.64064 & 1.5766 & $\mathrm{H}$ & 2.76013 & 0.37672 & -1.73119 \\
\hline $\mathrm{C}$ & 0.14695 & -0.96564 & 0.45533 & $\mathrm{H}$ & 1.35239 & 1.01096 & -2.57073 \\
\hline $\mathrm{C}$ & 0.43388 & 0.54425 & 0.48209 & $\mathrm{H}$ & 1.35563 & -1.48662 & -2.78224 \\
\hline $\mathrm{N}$ & 1.23014 & 1.08214 & -0.4867 & $\mathrm{H}$ & 0.12452 & -2.61843 & -1.01687 \\
\hline $\mathrm{C}$ & 1.94756 & 2.31768 & -0.15517 & $\mathrm{H}$ & -1.54002 & -0.77097 & 1.79313 \\
\hline $\mathrm{C}$ & 3.00158 & 2.0785 & 0.96562 & $\mathrm{H}$ & -1.50345 & -2.29539 & 0.90278 \\
\hline $\mathrm{O}$ & 0.04534 & 1.22023 & 1.43165 & $\mathrm{H}$ & -2.21156 & 0.4378 & -0.25667 \\
\hline $\mathrm{C}$ & 1.66315 & 0.40423 & -1.70762 & $\mathrm{H}$ & -2.15721 & -1.06767 & -1.18672 \\
\hline $\mathrm{C}$ & 1.13764 & -0.98297 & -1.84577 & $\mathrm{H}$ & -6.28243 & -2.19266 & 1.04024 \\
\hline $\mathrm{C}$ & 0.46588 & -1.59475 & -0.8786 & $\mathrm{H}$ & -7.26751 & -1.90095 & -0.40091 \\
\hline $\mathrm{C}$ & -1.33919 & -1.21543 & 0.81658 & $\mathrm{H}$ & -5.87205 & -2.98333 & -0.48272 \\
\hline $\mathrm{C}$ & -2.33705 & -0.64977 & -0.18849 & $\mathrm{H}$ & -4.21413 & 0.2588 & -2.68355 \\
\hline $\mathrm{O}$ & -3.65229 & -0.9766 & 0.24657 & $\mathrm{H}$ & -5.8284 & -0.42435 & -2.88697 \\
\hline $\mathrm{Si}$ & -5.11422 & -0.62228 & -0.50082 & $\mathrm{H}$ & -4.43478 & -1.49324 & -2.73614 \\
\hline $\mathrm{C}$ & -5.80184 & 1.04233 & 0.16276 & $\mathrm{H}$ & -7.89382 & 0.61391 & -0.3829 \\
\hline $\mathrm{C}$ & -6.24476 & -2.05342 & -0.04331 & $\mathrm{H}$ & -7.53771 & 2.32498 & -0.14806 \\
\hline $\mathrm{C}$ & -4.87188 & -0.55778 & -2.37294 & $\mathrm{H}$ & -7.00629 & 1.51343 & -1.62131 \\
\hline $\mathrm{C}$ & -7.13244 & 1.38404 & -0.54181 & $\mathrm{H}$ & -6.3997 & 1.88987 & 2.08187 \\
\hline $\mathrm{C}$ & -6.04816 & 0.93002 & 1.68225 & $\mathrm{H}$ & -6.80972 & 0.18052 & 1.91774 \\
\hline $\mathrm{C}$ & -4.79225 & 2.18173 & -0.09275 & $\mathrm{H}$ & -5.13467 & 0.66148 & 2.22024 \\
\hline $\mathrm{H}$ & 7.6101 & 1.55521 & -1.83821 & $\mathrm{H}$ & -3.84965 & 2.01611 & 0.43616 \\
\hline $\mathrm{H}$ & 8.10989 & -0.86079 & -1.7094 & $\mathrm{H}$ & -4.56766 & 2.30533 & -1.15706 \\
\hline $\mathrm{H}$ & 6.62377 & -2.40119 & -0.45532 & $\mathrm{H}$ & -5.2012 & 3.13551 & 0.26467 \\
\hline
\end{tabular}


Table S8. Optimized Cartesian Coordinates and Zero Point Vibrational Energy Corrected Absolute Energy in Hartree of vinylogous urethane (-)-8: $E=-1676.88643626$ a.u.

\begin{tabular}{|c|c|c|c|c|c|c|c|}
\hline $\mathrm{C}$ & 1.13867 & -3.82501 & -2.21667 & $\mathrm{H}$ & 1.3325 & -3.65464 & -0.07294 \\
\hline $\mathrm{C}$ & 1.29871 & -3.1901 & -3.44724 & $\mathrm{H}$ & 2.47287 & 0.84235 & -2.82794 \\
\hline $\mathrm{C}$ & 1.76728 & -1.87447 & -3.52808 & $\mathrm{H}$ & 2.96713 & 1.56305 & 1.86974 \\
\hline $\mathrm{C}$ & 2.06038 & -1.21975 & -2.33897 & $\mathrm{H}$ & 1.6748 & 2.68502 & 1.48213 \\
\hline $\mathrm{C}$ & 1.90963 & -1.84692 & -1.09054 & $\mathrm{H}$ & 0.89887 & -1.56145 & 1.36103 \\
\hline $\mathrm{C}$ & 1.45386 & -3.15296 & -1.02682 & $\mathrm{H}$ & 3.28139 & -3.01427 & 1.68112 \\
\hline $\mathrm{N}$ & 2.51733 & 0.09145 & -2.14753 & $\mathrm{H}$ & 4.58056 & -2.01784 & 2.37312 \\
\hline $\mathrm{C}$ & 2.49223 & 0.40761 & -0.81035 & $\mathrm{H}$ & 4.53427 & -0.47633 & 0.50053 \\
\hline $\mathrm{C}$ & 2.43498 & -0.89238 & -0.02805 & $\mathrm{H}$ & 4.32447 & -2.02649 & -0.33737 \\
\hline $\mathrm{C}$ & 2.41403 & 1.63842 & -0.23347 & $\mathrm{H}$ & 1.66265 & -2.28742 & 3.74662 \\
\hline $\mathrm{C}$ & 2.08001 & 1.70086 & 1.24459 & $\mathrm{H}$ & 3.01138 & -1.34018 & 4.38171 \\
\hline $\mathrm{C}$ & 1.04717 & 0.59542 & 1.64124 & $\mathrm{H}$ & 0.96022 & -0.10035 & 5.08195 \\
\hline $\mathrm{C}$ & 1.68066 & -0.78444 & 1.33623 & $\mathrm{H}$ & 0.12075 & 1.48079 & 3.45586 \\
\hline $\mathrm{N}$ & 2.70741 & -1.06069 & 2.34489 & $\mathrm{H}$ & -0.11173 & 0.77968 & -0.20418 \\
\hline $\mathrm{C}$ & 3.66769 & -1.98062 & 1.77146 & $\mathrm{H}$ & -0.6214 & 1.84816 & 1.08379 \\
\hline $\mathrm{C}$ & 3.88862 & -1.35031 & 0.39775 & $\mathrm{H}$ & -1.18903 & -1.14861 & 0.94103 \\
\hline $\mathrm{C}$ & 2.18036 & -1.3073 & 3.66756 & $\mathrm{H}$ & -1.65671 & -0.09132 & 2.28107 \\
\hline $\mathrm{C}$ & 1.23704 & -0.19323 & 4.03603 & $\mathrm{H}$ & -4.97339 & -2.6781 & -0.01191 \\
\hline $\mathrm{C}$ & 0.7693 & 0.66905 & 3.13301 & $\mathrm{H}$ & -3.41569 & -2.76729 & 0.80248 \\
\hline $\mathrm{C}$ & -0.28279 & 0.82744 & 0.87331 & $\mathrm{H}$ & -3.4853 & -2.36383 & -0.91565 \\
\hline $\mathrm{C}$ & -1.43921 & -0.11392 & 1.20593 & $\mathrm{H}$ & -5.85611 & -0.65265 & 2.26287 \\
\hline $\mathrm{O}$ & -2.579 & 0.30995 & 0.46322 & $\mathrm{H}$ & -4.9043 & 0.81076 & 2.54712 \\
\hline $\mathrm{Si}$ & -4.10668 & -0.38591 & 0.49317 & $\mathrm{H}$ & -4.2429 & -0.7744 & 2.95826 \\
\hline $\mathrm{C}$ & -5.1061 & 0.60313 & -0.80173 & $\mathrm{H}$ & -5.02242 & 1.01363 & -2.94101 \\
\hline $\mathrm{C}$ & -3.98325 & -2.21571 & 0.04634 & $\mathrm{H}$ & -4.48714 & -0.61634 & -2.52698 \\
\hline $\mathrm{C}$ & -4.84651 & -0.2321 & 2.22354 & $\mathrm{H}$ & -3.43015 & 0.76603 & -2.20862 \\
\hline $\mathrm{C}$ & -4.46915 & 0.42686 & -2.19664 & $\mathrm{H}$ & -4.07733 & 2.49611 & -0.39027 \\
\hline $\mathrm{C}$ & -5.09585 & 2.10241 & -0.43453 & $\mathrm{H}$ & -5.64761 & 2.67913 & -1.18791 \\
\hline $\mathrm{C}$ & -6.56538 & 0.10157 & -0.84289 & $\mathrm{H}$ & -5.57281 & 2.29178 & 0.53228 \\
\hline $\mathrm{C}$ & 2.46041 & 2.81563 & -1.0881 & $\mathrm{H}$ & -6.63122 & -0.95664 & -1.1153 \\
\hline $\mathrm{O}$ & 2.60956 & 2.80192 & -2.30637 & $\mathrm{H}$ & -7.13606 & 0.66458 & -1.59207 \\
\hline $\mathrm{O}$ & 2.31304 & 3.9806 & -0.40742 & $\mathrm{H}$ & -7.07402 & 0.23356 & 0.11733 \\
\hline $\mathrm{C}$ & 2.33473 & 5.17813 & -1.19912 & $\mathrm{H}$ & 2.21274 & 5.99562 & -0.49109 \\
\hline $\mathrm{H}$ & 0.76752 & -4.84235 & -2.17847 & $\mathrm{H}$ & 3.28287 & 5.27062 & -1.73113 \\
\hline $\mathrm{H}$ & 1.05182 & -3.72035 & -4.36013 & $\mathrm{H}$ & 1.51964 & 5.17579 & -1.92462 \\
\hline $\mathrm{H}$ & 1.88531 & -1.38189 & -4.48633 & & & & \\
\hline
\end{tabular}




\section{References}

${ }^{1}$ Still, W. C.; Kahn, M.; Mitra, A. J. Org. Chem. 1978, 43, 2923.

${ }^{2}$ Pangborn, A. B.; Giardello, M. A.; Grubbs, R. H.; Rosen, R. K.; Timmers, F. Organometallics 1996, $15,1518$.

${ }^{3}$ Lipton, M. F.; Sorensen, C. M.; Sadler, A. C.; Shapiro, R. H. J. Organomet. Chem. 1980, 186, 155.

${ }^{4}$ a) Gottlieb, H. E.; Kotlyar, V.; Nudelman, A. J. Org. Chem. 1997, 62, 7512; b) Fulmer, G. R.; Miller, A. J. M.; Sherden, N. H.; Gottlieb, H. E.; Nudelman, A.; Stoltz, B. M.; Bercaw, J. E.; Goldberg, K. I. Organometallics 2010, 29, 2176.

${ }^{5}$ Saxton, J. E. Alkaloids of the Aspidospermine Group. In The Alkaloids, Chemistry and Biology, Vol 51 (Ed.: Cordell, G. A.), Academic Press, San Diego, 1998, pp 1-197.

${ }^{6}$ Lee, K.; Boger, D. L. J. Am. Chem. Soc. 2014, 136, 3312.

7 a) Mewald, M.; Medley, J. W.; Movassaghi, M. Angew. Chem. Int. Ed. 2014, 53, 11634-11639; b) White, K. L.; Movassaghi, M. J. Am. Chem. Soc. 2016, 138, 11383-11389.

${ }^{8}$ Kam, T.-S.; Choo, Y.-M. Helv. Chim. Acta 2004, 87, 991-998.

${ }^{9}$ Zhou, Y.-G.; Wong, H. N. C.; Peng, X.-S. J. Org. Chem. 2020, 85, 967-976.

${ }^{10}$ The solvent reference value was not provided in reference 7 .

${ }^{11}$ The solvent reference value was not provided in reference 6 .

${ }^{12}$ This data point is most consistent with prior synthetic samples.

${ }^{13}$ Frisch, M. J.; Trucks, G. W.; Schlegel, H. B.; Scuseria, G. E.; Robb, M. A.; Cheeseman, J. R.; Scalmani, G.; Barone, V.; Mennucci, B.; Petersson, G. A.; Nakatsuji, H.; Caricato, M.; Li, X.; Hratchian, H. P.; Izmaylov, A. F.; Bloino, J.; Zheng, G.; Sonnenberg, J. L.; Hada, M.; Ehara, M.; Toyota, K.; Fukuda, R.; Hasegawa, J.; Ishida, M.; Nakajima, T.; Honda, Y.; Kitao, O.; Nakai, H.; Vreven, T.; Montgomery Jr., J. A.; Peralta, J. E.; Ogliaro, F.; Bearpark, M.; Heyd, J. J.; Brothers, E.; Kudin, K. N.; Staroverov, N.; Keith, T. V.; Kobayashi, R.; Normand, J.; Raghavachari, K.; Rendell, A.; Burant, J. C.; Iyengar, S. S.; Tomasi, J.; Cossi, M.; Rega, N.; Millam, J. M.; Klene, M.; Knox, J. E.; Cross, J. B.; Bakken, V.; Adamo, C.; Jaramillo, J.; Gomperts, R.; Stratmann, R. E.; Yazyev, O.; Austin, A. J.; Cammi, R.; Pomelli, C.; Ochterski, J. W.; Martin, R. L.; Morokuma, K.; Zakrzewski, V. G.; Voth, G. A.; Salvador, P.; Dannenberg, J. J.; Dapprich, S.; Daniels, A. D.; Farkas, O.; Foresman, J. B.; Ortiz, J. V.; Cioslowski, J.; Fox, D. J. Gaussian 09, Revision D.01; Gaussian, Inc.: Wallingford, CT, 2013.

${ }^{14}$ Halgren, T. A. Merck molecular force field. I. Basis, form, scope, parameterization, and performance of MMFF94. J. Comput. Chem. 1996, 17, 490-519. 
규슨 (t)

${ }^{1} \mathrm{H} \mathrm{NMR}, 600 \mathrm{MHz}, \mathrm{CDCl}_{3}, 25^{\circ} \mathrm{C}$

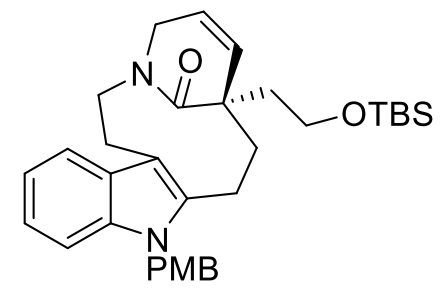

$(+)-10$

I I I
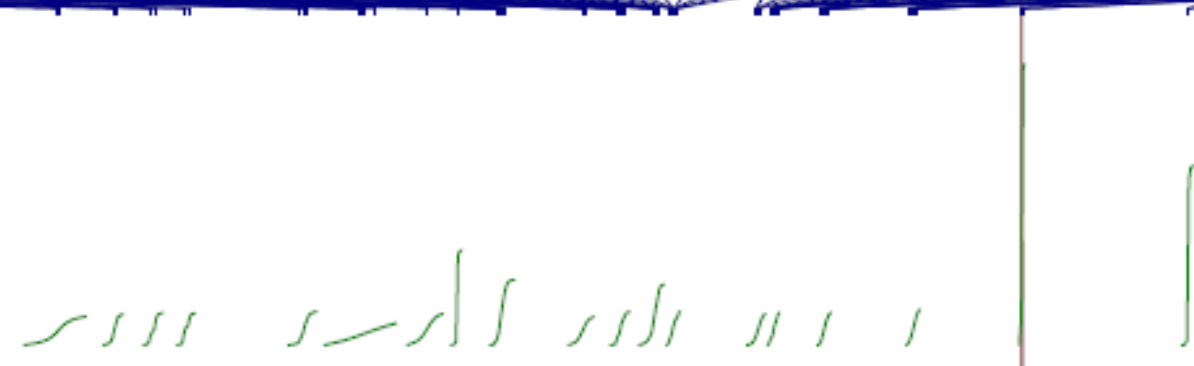

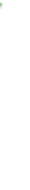

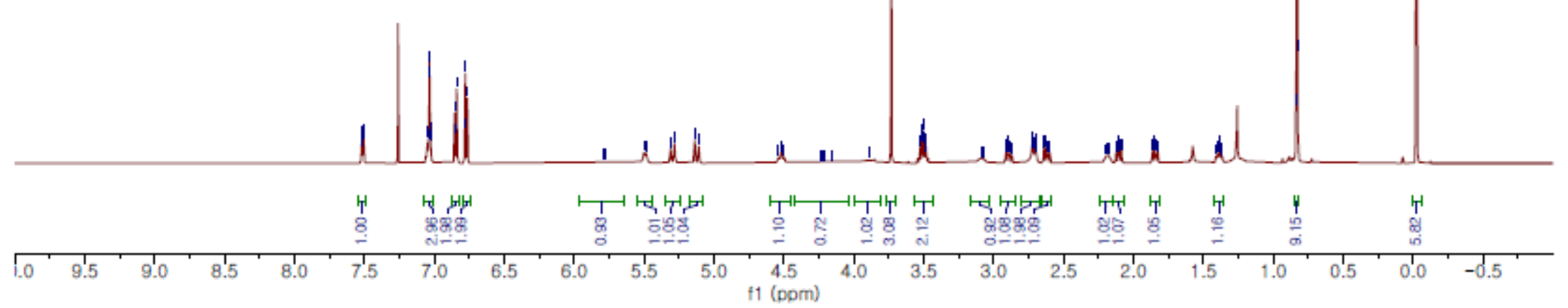


${ }^{13} \mathrm{C}$ NMR, $150.9 \mathrm{MHz}, \mathrm{CDCl}_{3}, 25^{\circ} \mathrm{C}$

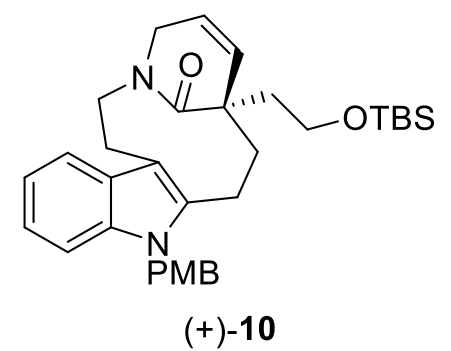

$(+)-10$
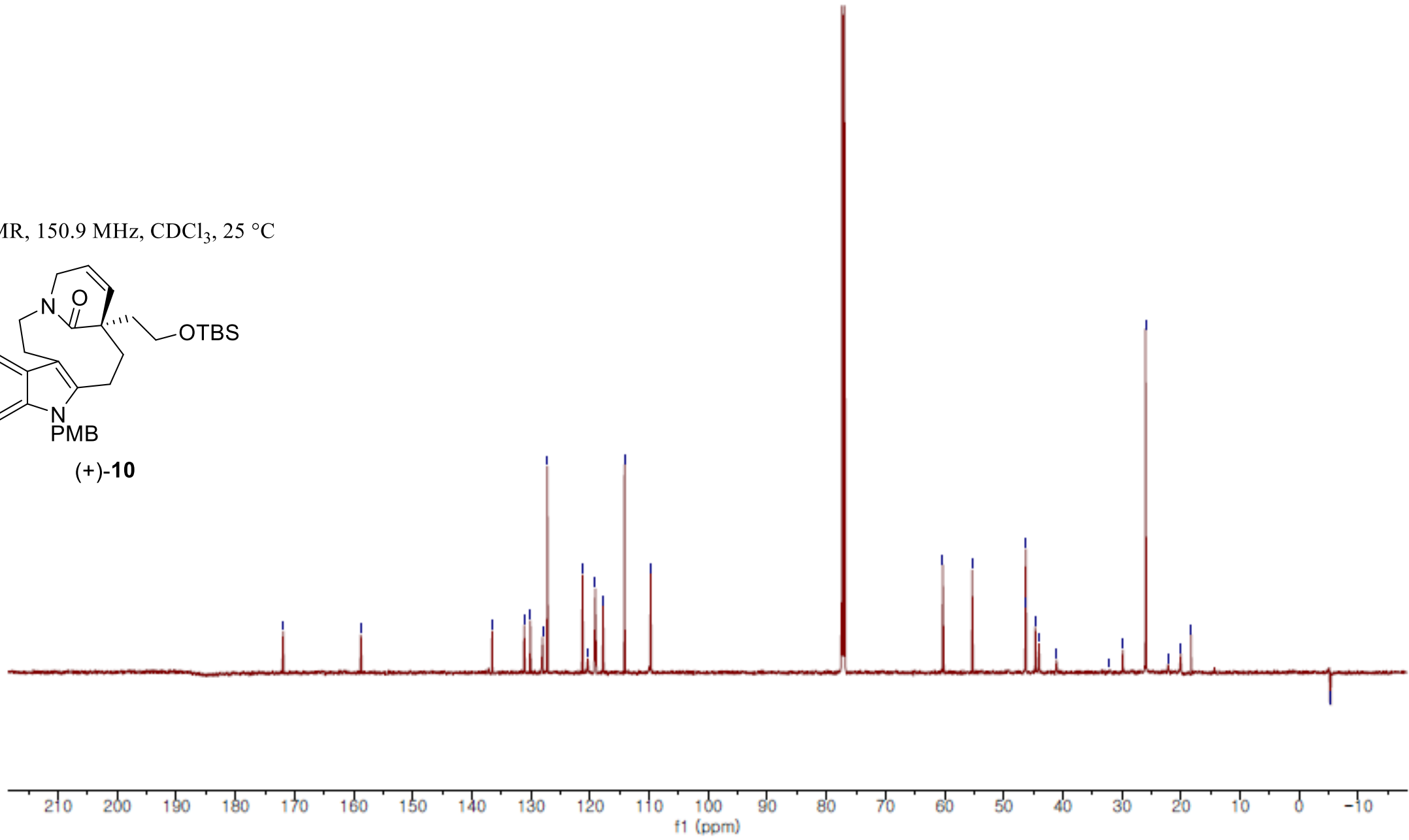


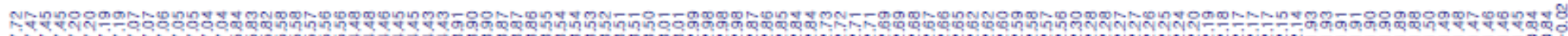

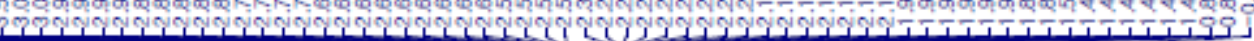

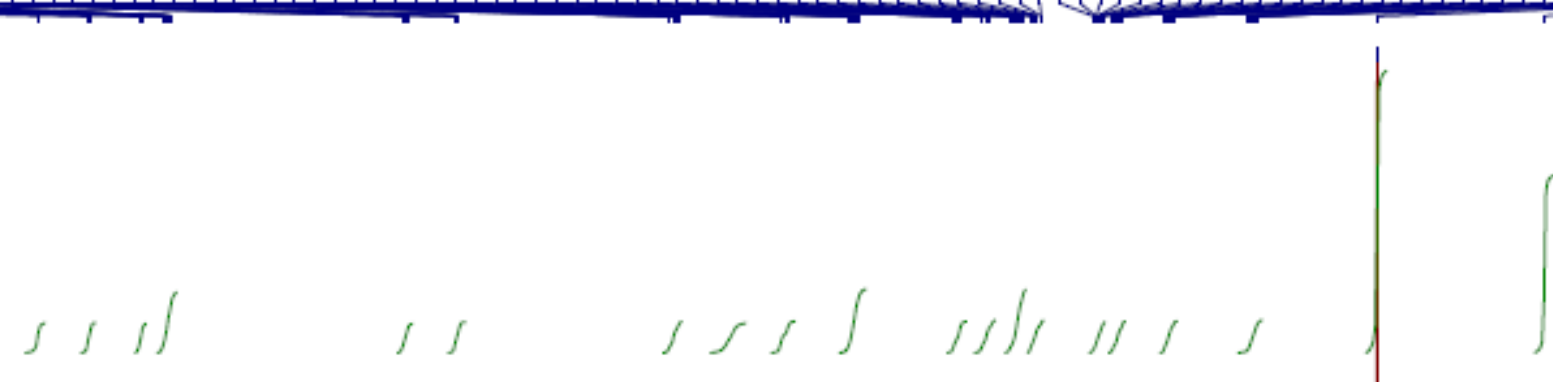

${ }^{1} \mathrm{H} \mathrm{NMR}, 500 \mathrm{MHz}, \mathrm{CDCl}_{3}, 25^{\circ} \mathrm{C}$

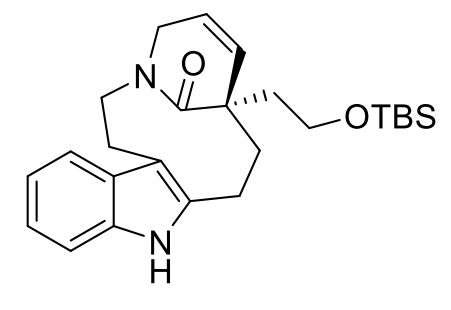

$(+)-11$

$\mu$

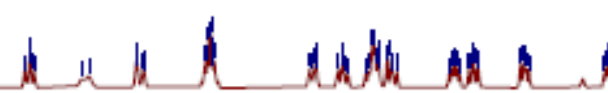

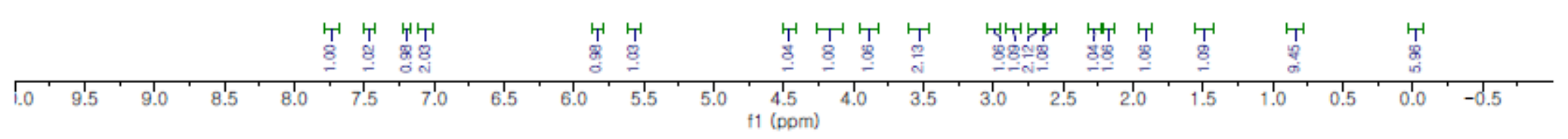



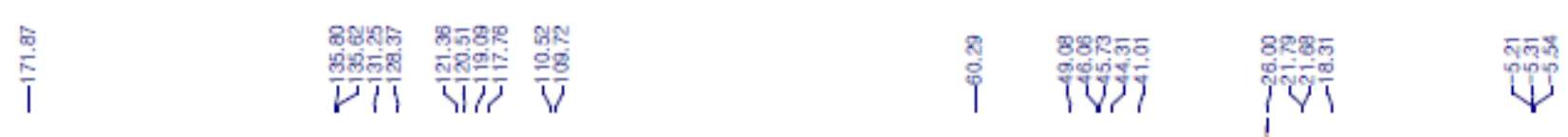

${ }^{13} \mathrm{C}$ NMR, $125.8 \mathrm{MHz}, \mathrm{CDCl}_{3}, 25^{\circ} \mathrm{C}$

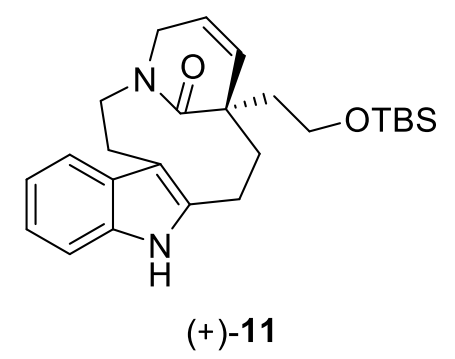

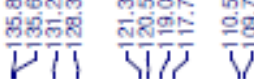

मूलि
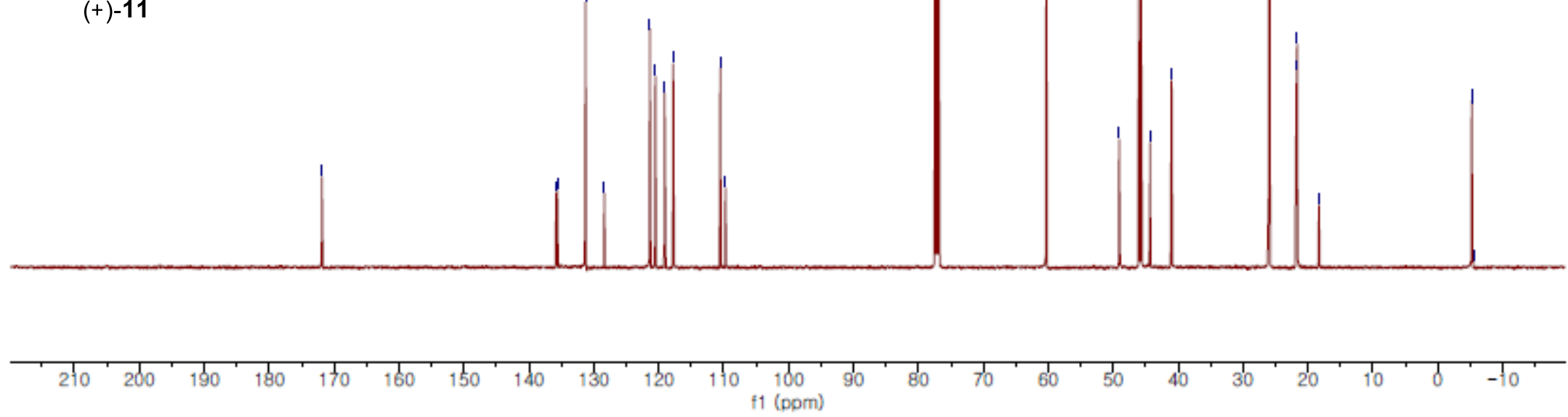


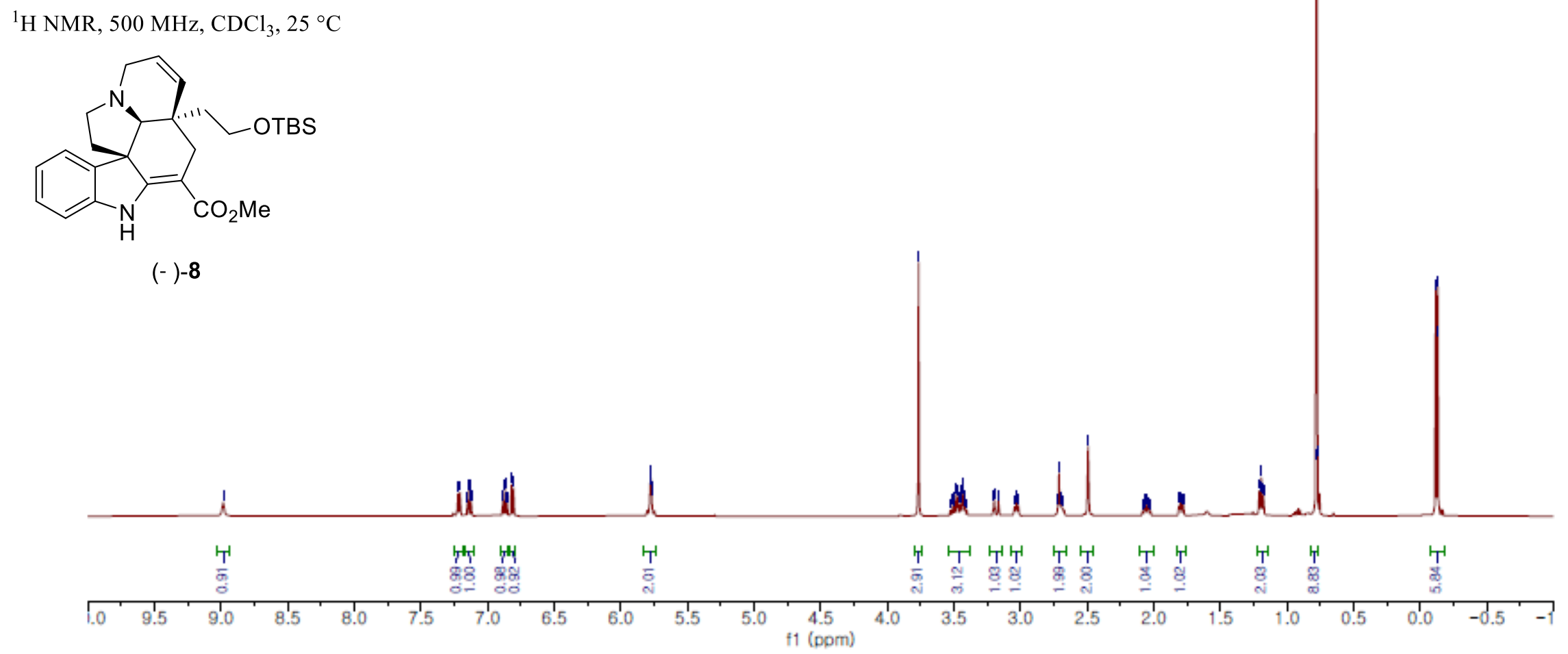




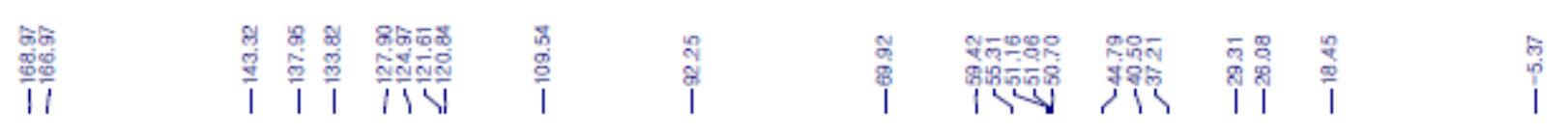

${ }^{13} \mathrm{C}$ NMR, $125.8 \mathrm{MHz}, \mathrm{CDCl}_{3}, 25{ }^{\circ} \mathrm{C}$

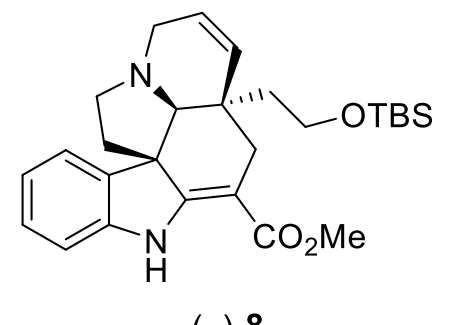

(-)-8 
9: W

${ }^{1} \mathrm{H} \mathrm{NMR}, 500 \mathrm{MHz}, \mathrm{CDCl}_{3}, 25^{\circ} \mathrm{C}$

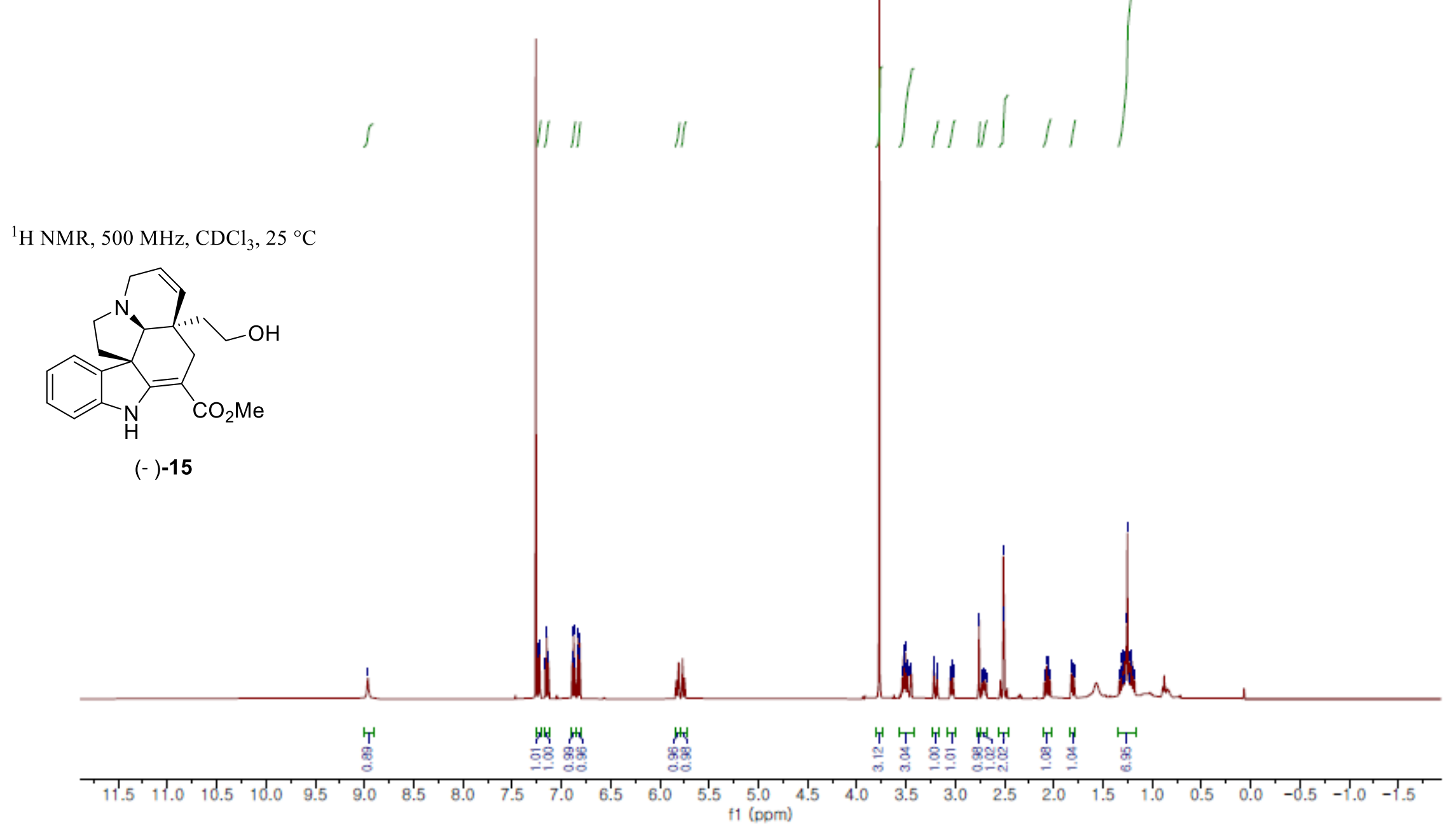

$(-)-15$

(1) 
${ }^{13} \mathrm{C}$ NMR, $125.8 \mathrm{MHz}, \mathrm{CDCl}_{3}, 25{ }^{\circ} \mathrm{C}$

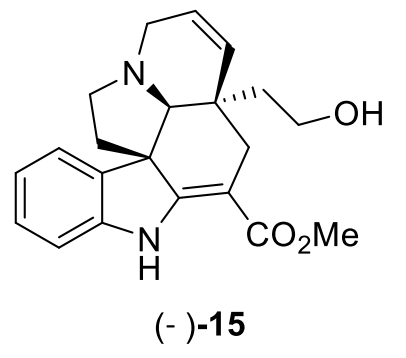

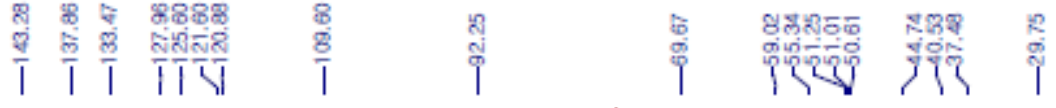

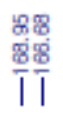

\section{1}
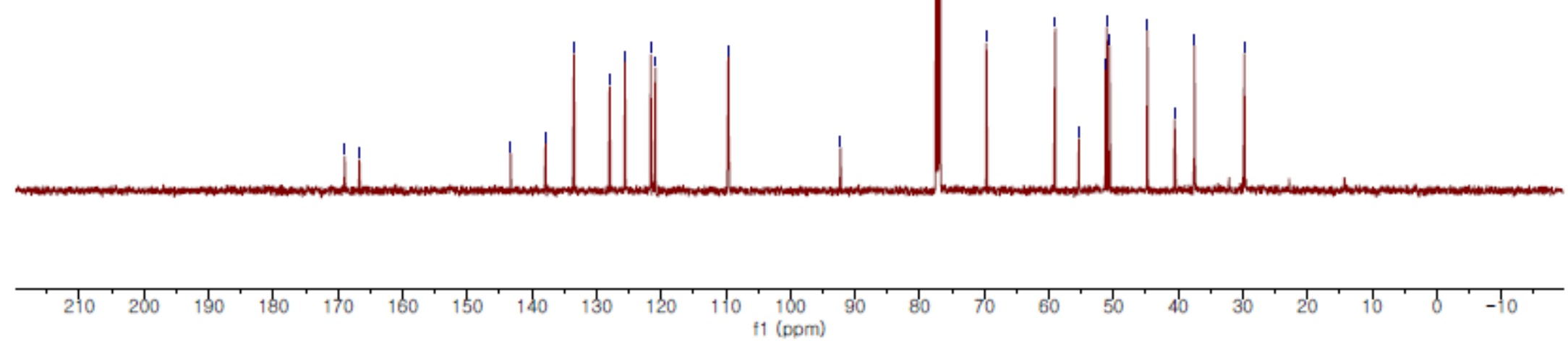


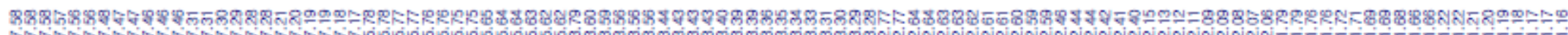

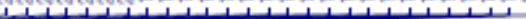

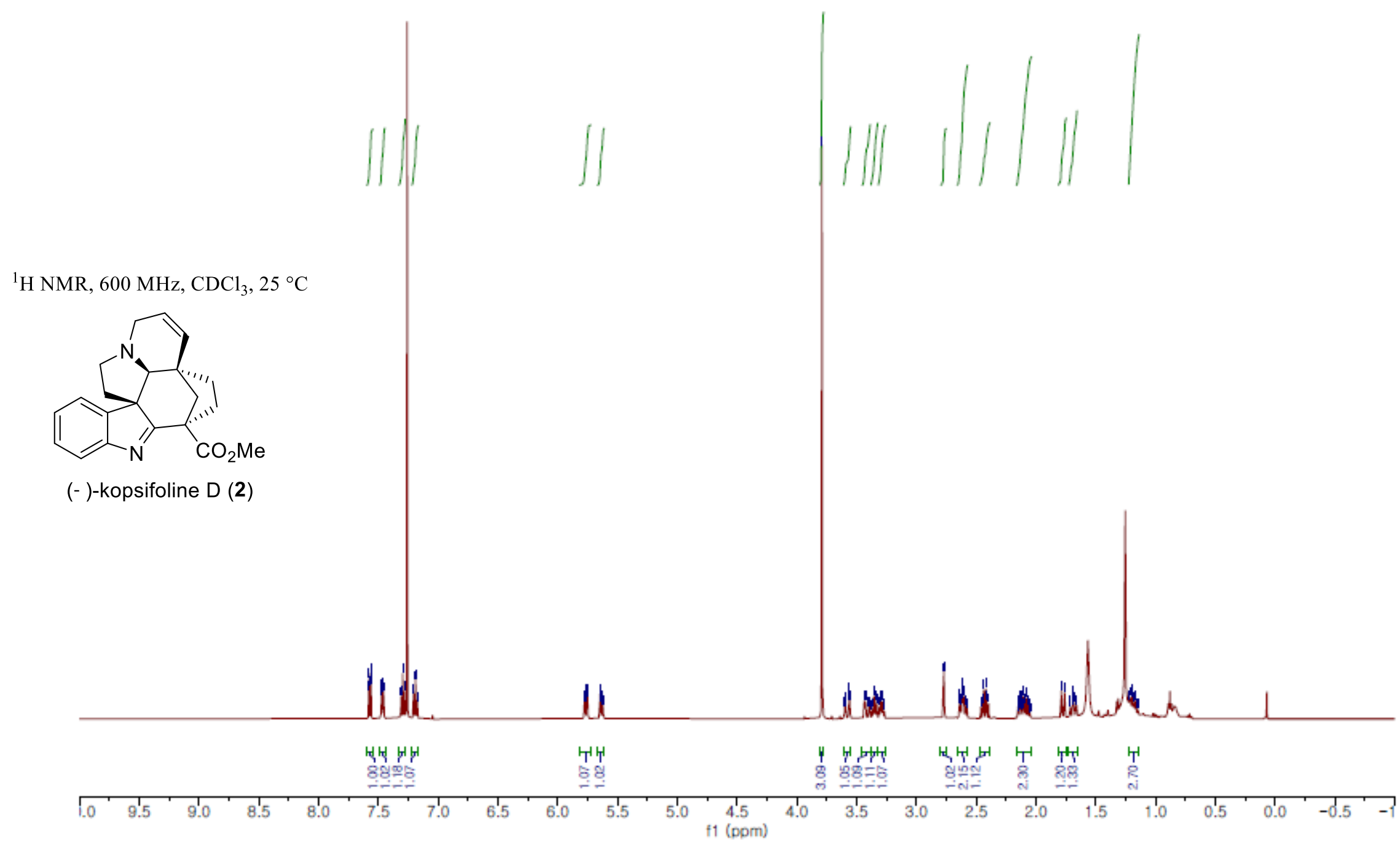


${ }^{13} \mathrm{C}$ NMR, $150.9 \mathrm{MHz}, \mathrm{CDCl}_{3}, 25{ }^{\circ} \mathrm{C}$

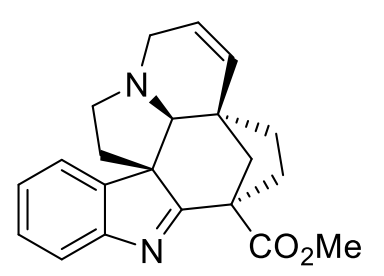

(- )-kopsifoline D (2)

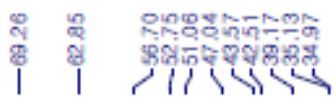

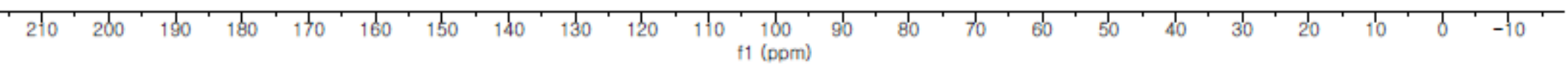



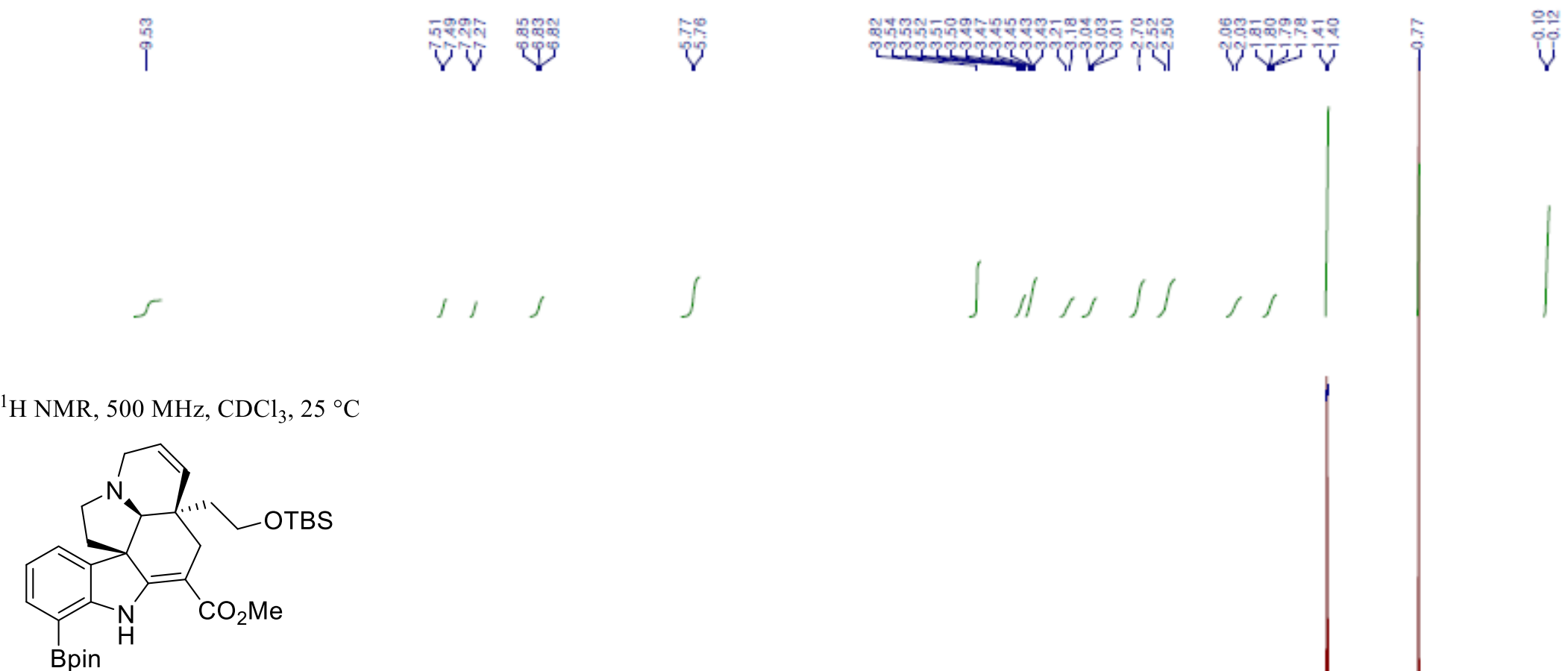

$(-)-12$

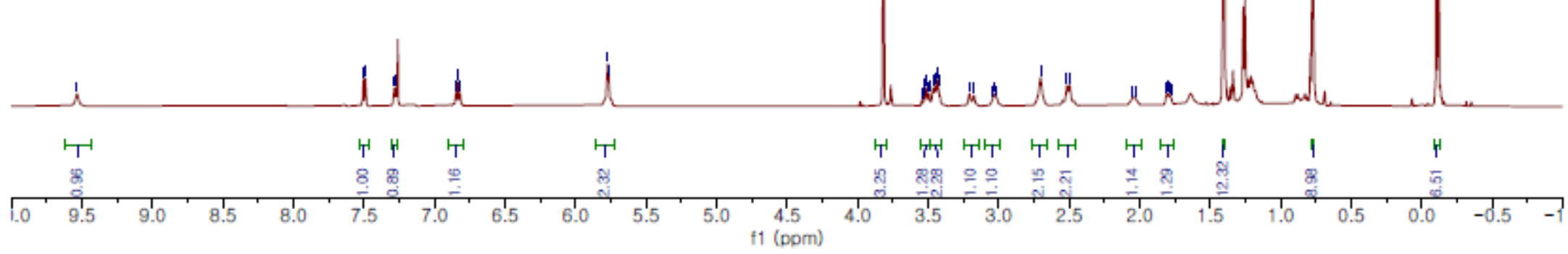



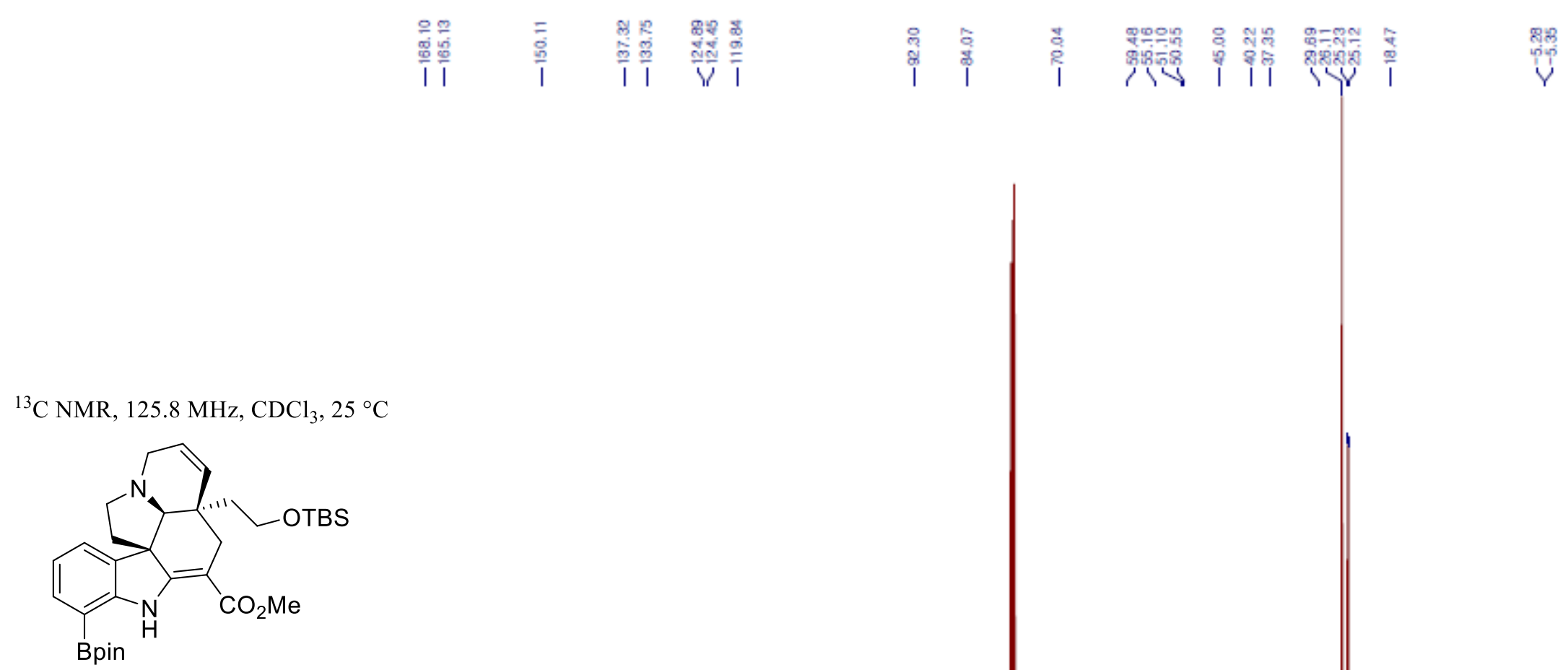

(- )-12
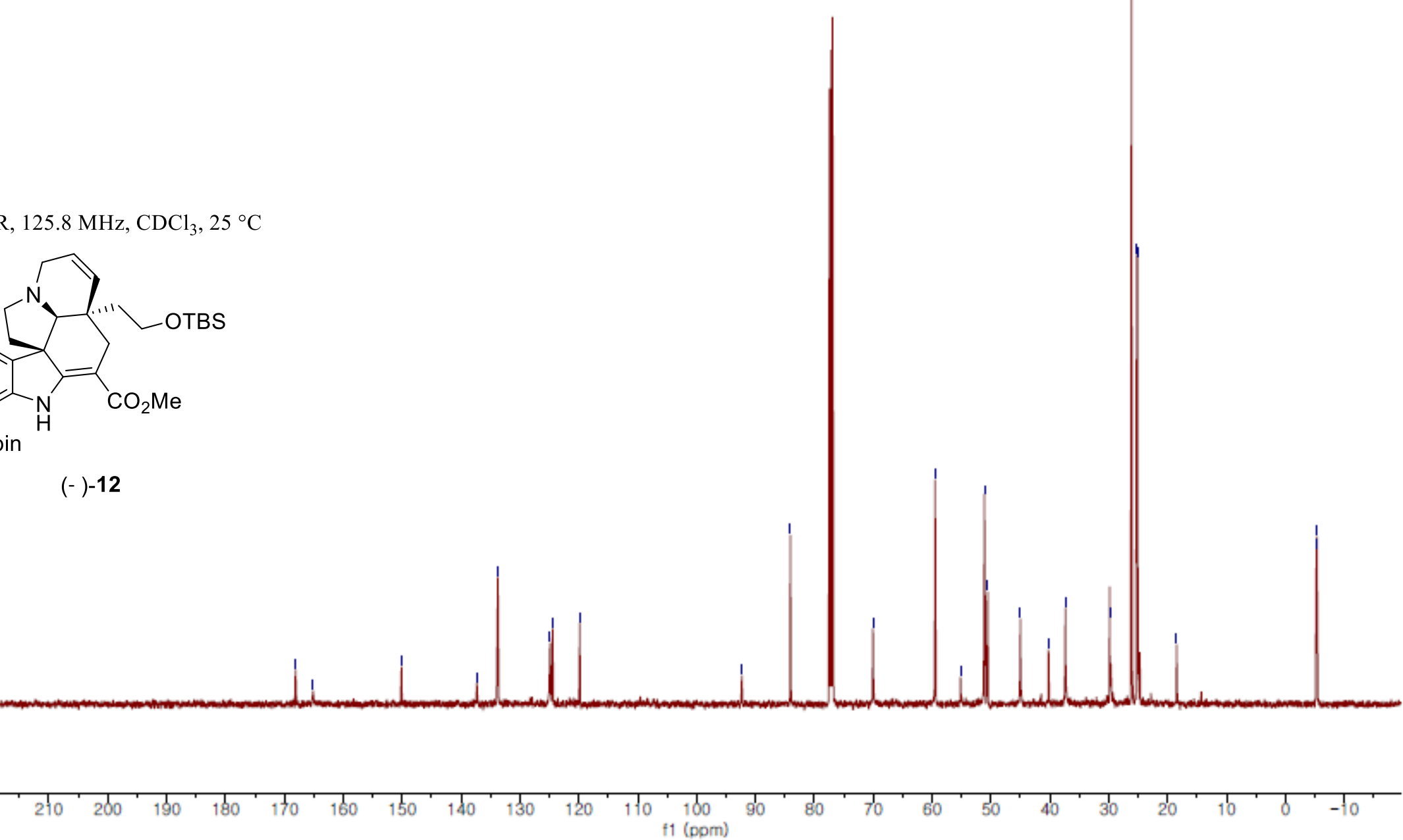
\% D.

\%
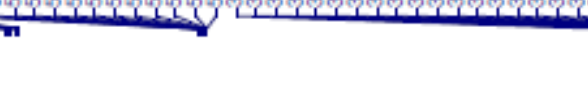

$\int$

III

$1 / 110111$

${ }^{1} \mathrm{H} \mathrm{NMR}, 600 \mathrm{MHz}, \mathrm{CD}_{3} \mathrm{CN}, 25^{\circ} \mathrm{C}$

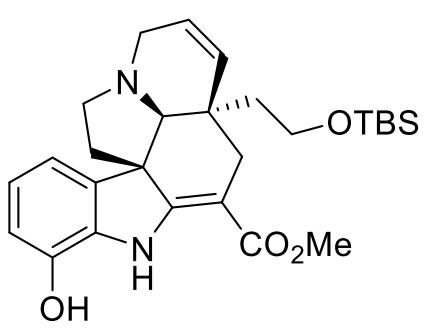

(- )-16
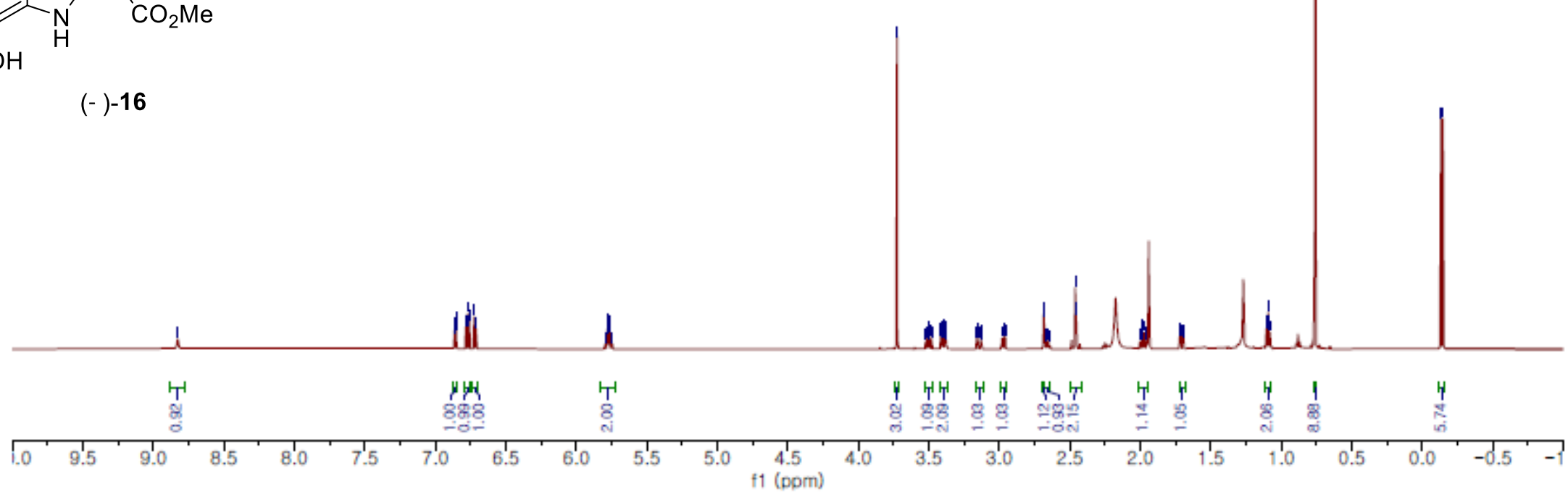
${ }^{13} \mathrm{C}$ NMR, $150.9 \mathrm{MHz}, \mathrm{CD}_{3} \mathrm{CN}, 25^{\circ} \mathrm{C}$

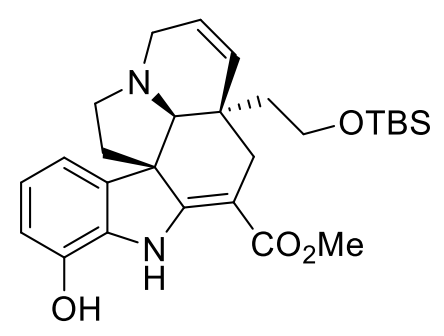

(- )-16
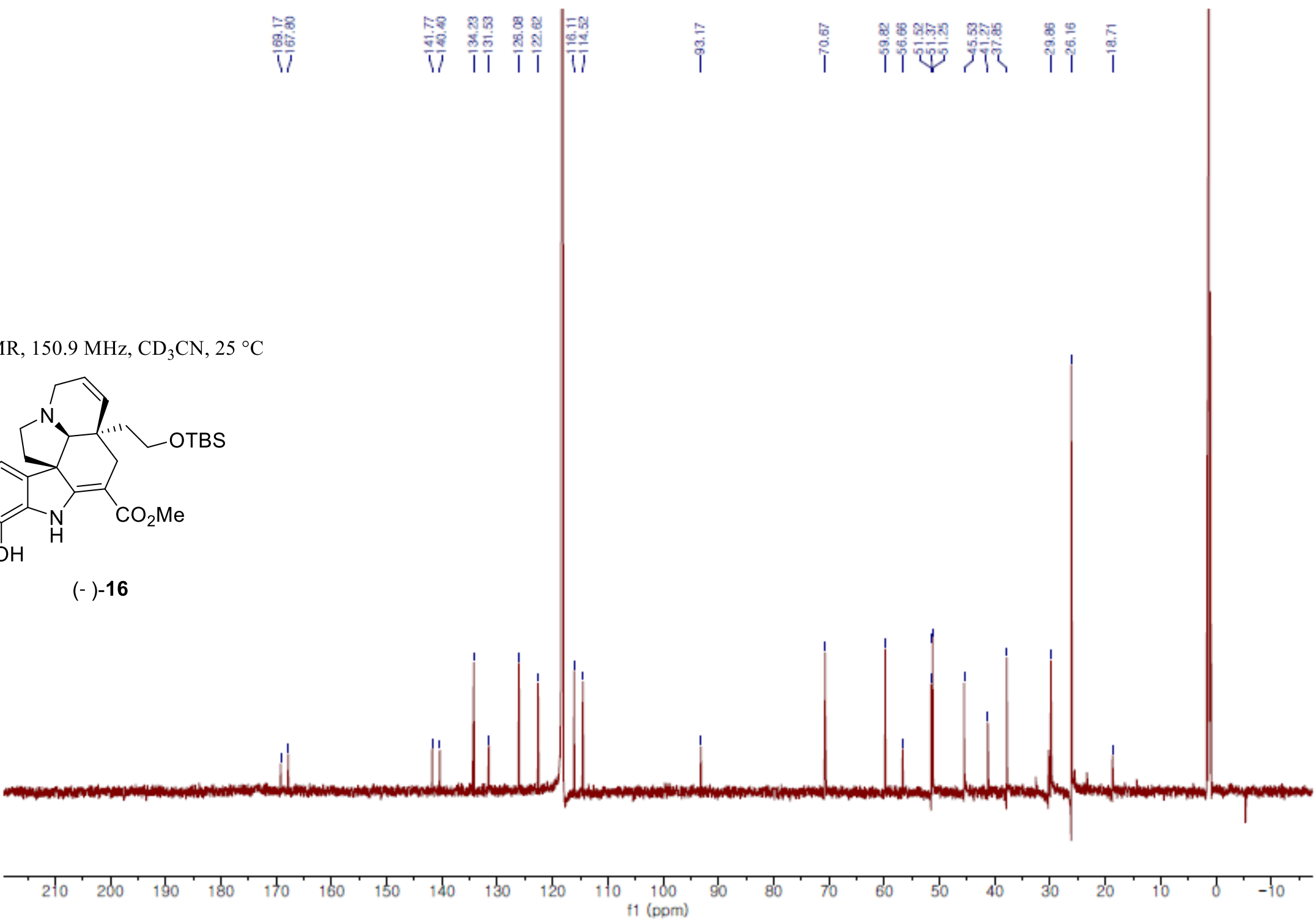


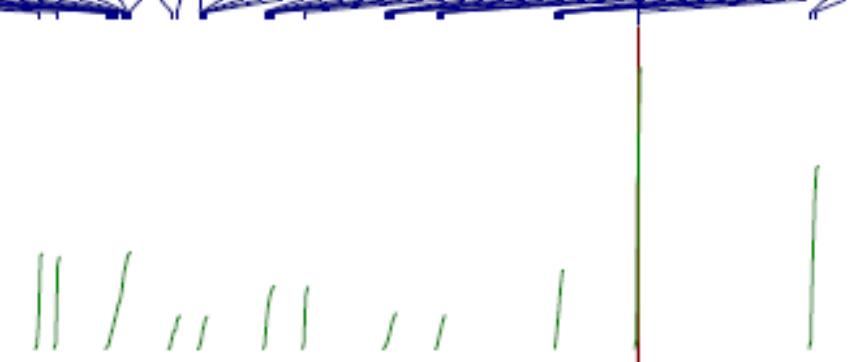

${ }^{1} \mathrm{H} \mathrm{NMR}, 600 \mathrm{MHz}, \mathrm{CDCl}_{3}, 25^{\circ} \mathrm{C}$

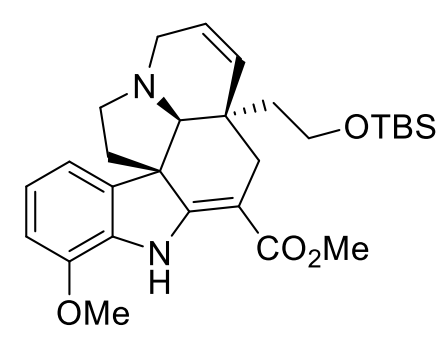

$(-)-7$
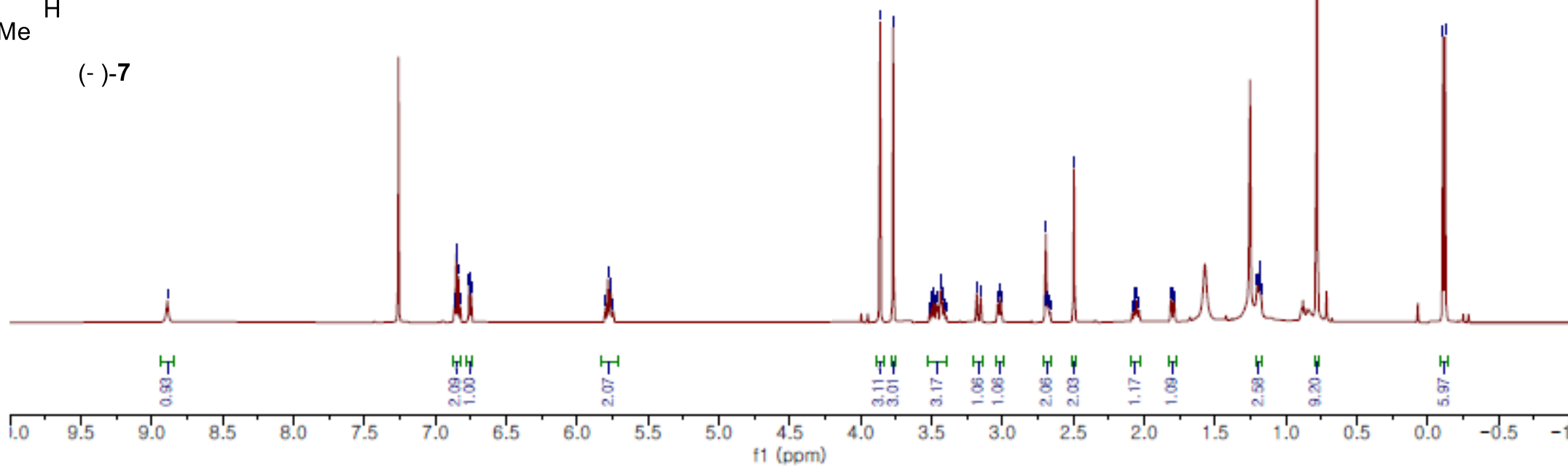


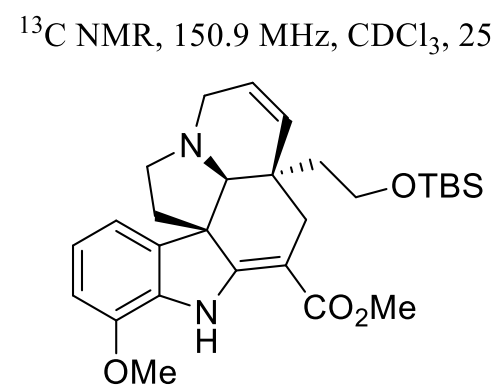

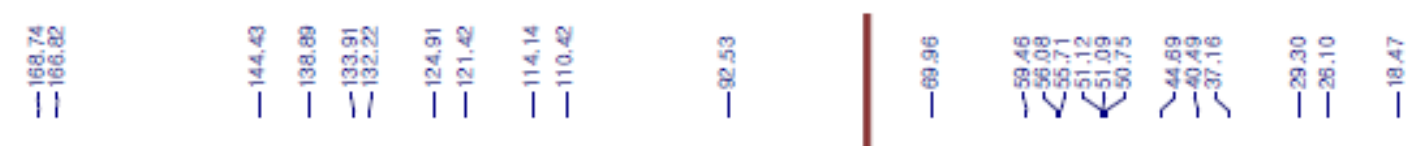

(-) - -7
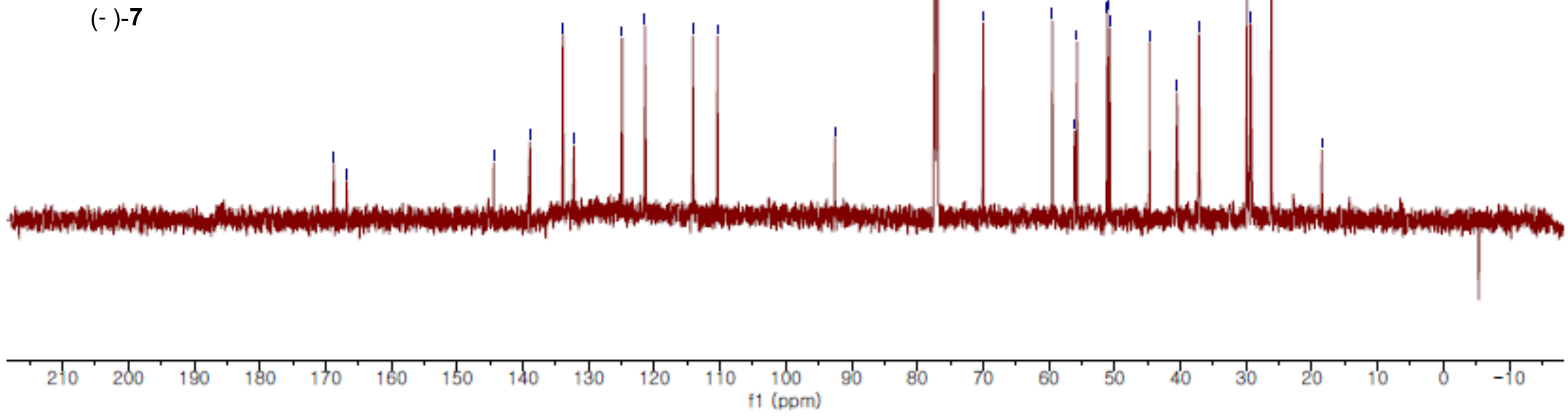
फ0 अ8.
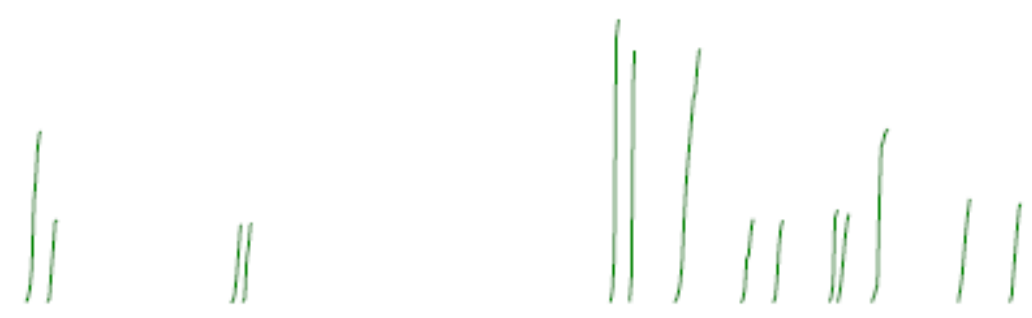

${ }^{1} \mathrm{H} \mathrm{NMR}, 600 \mathrm{MHz}, \mathrm{CDCl}_{3}, 25^{\circ} \mathrm{C}$

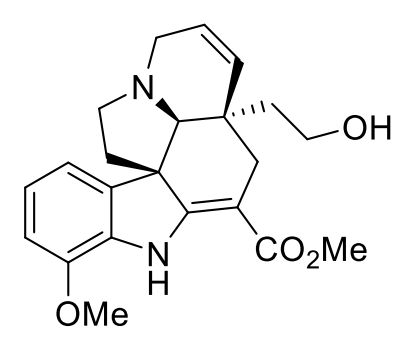

$(-)-17$
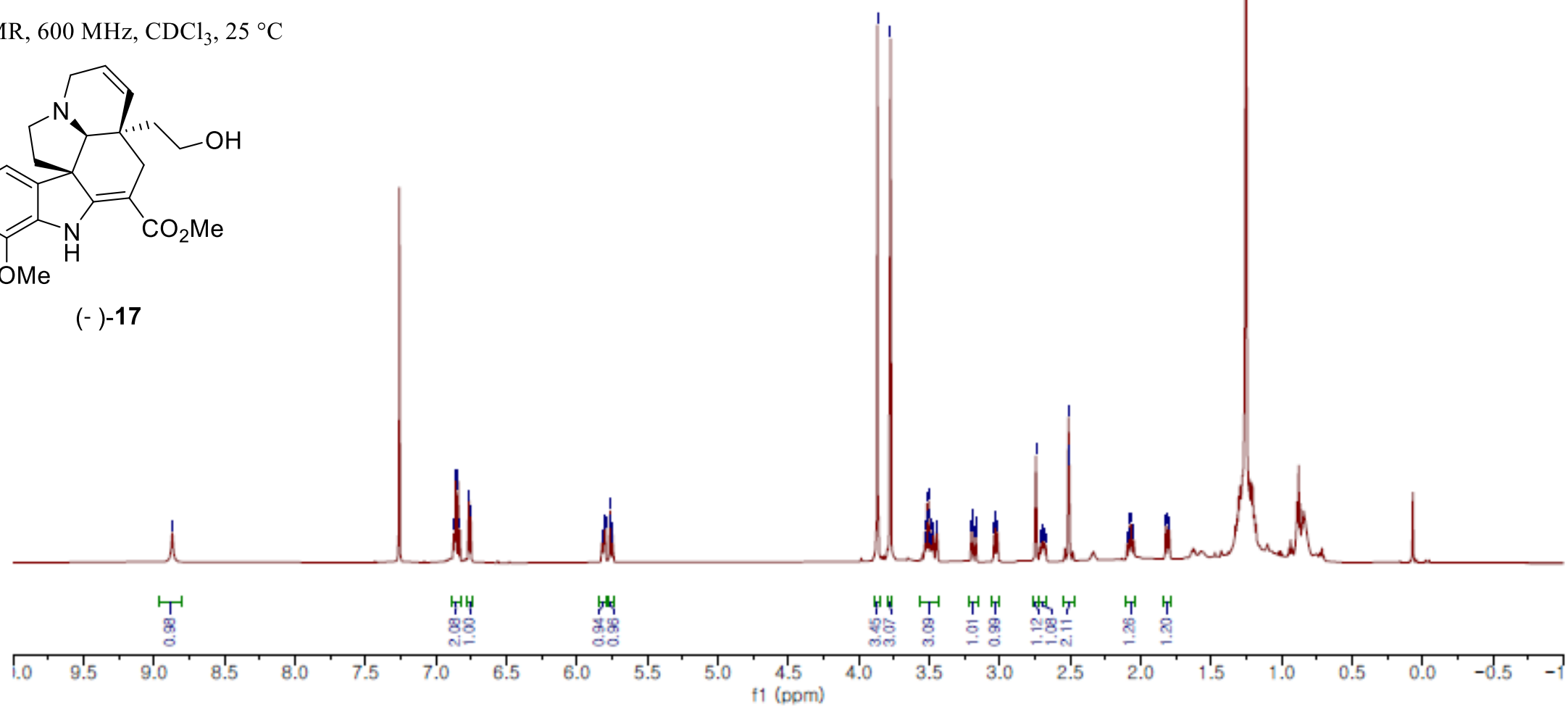
${ }^{13} \mathrm{C}$ NMR, $150.9 \mathrm{MHz}, \mathrm{CDCl}_{3}, 25{ }^{\circ} \mathrm{C}$

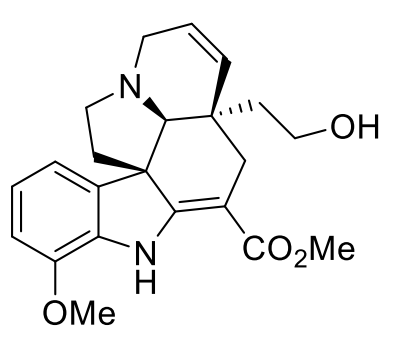

(- )-17

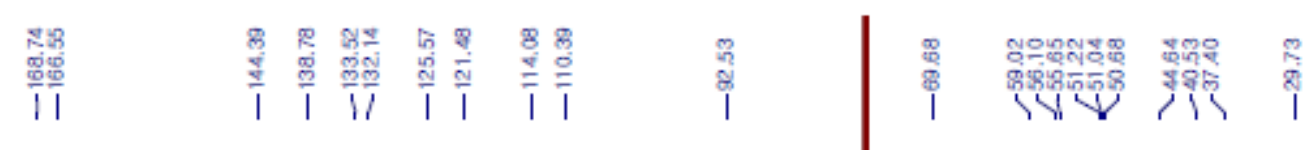
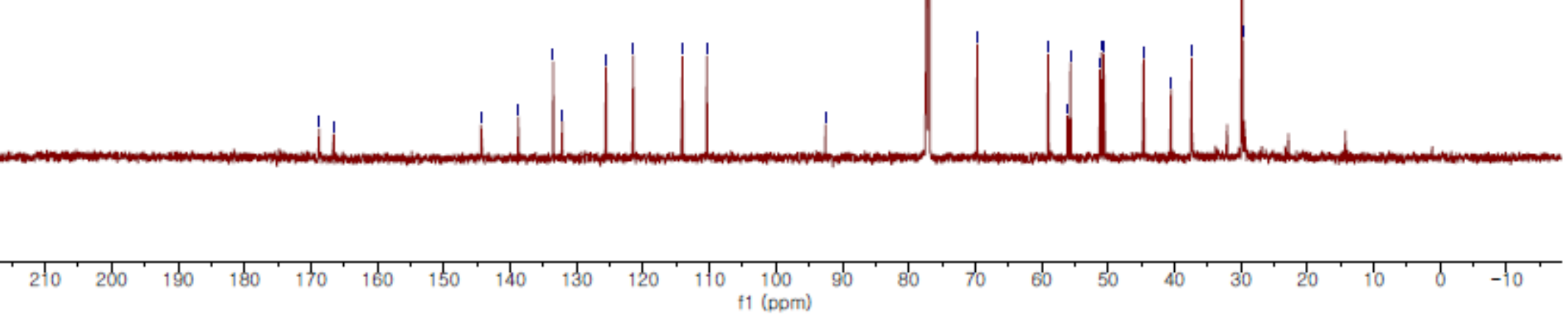


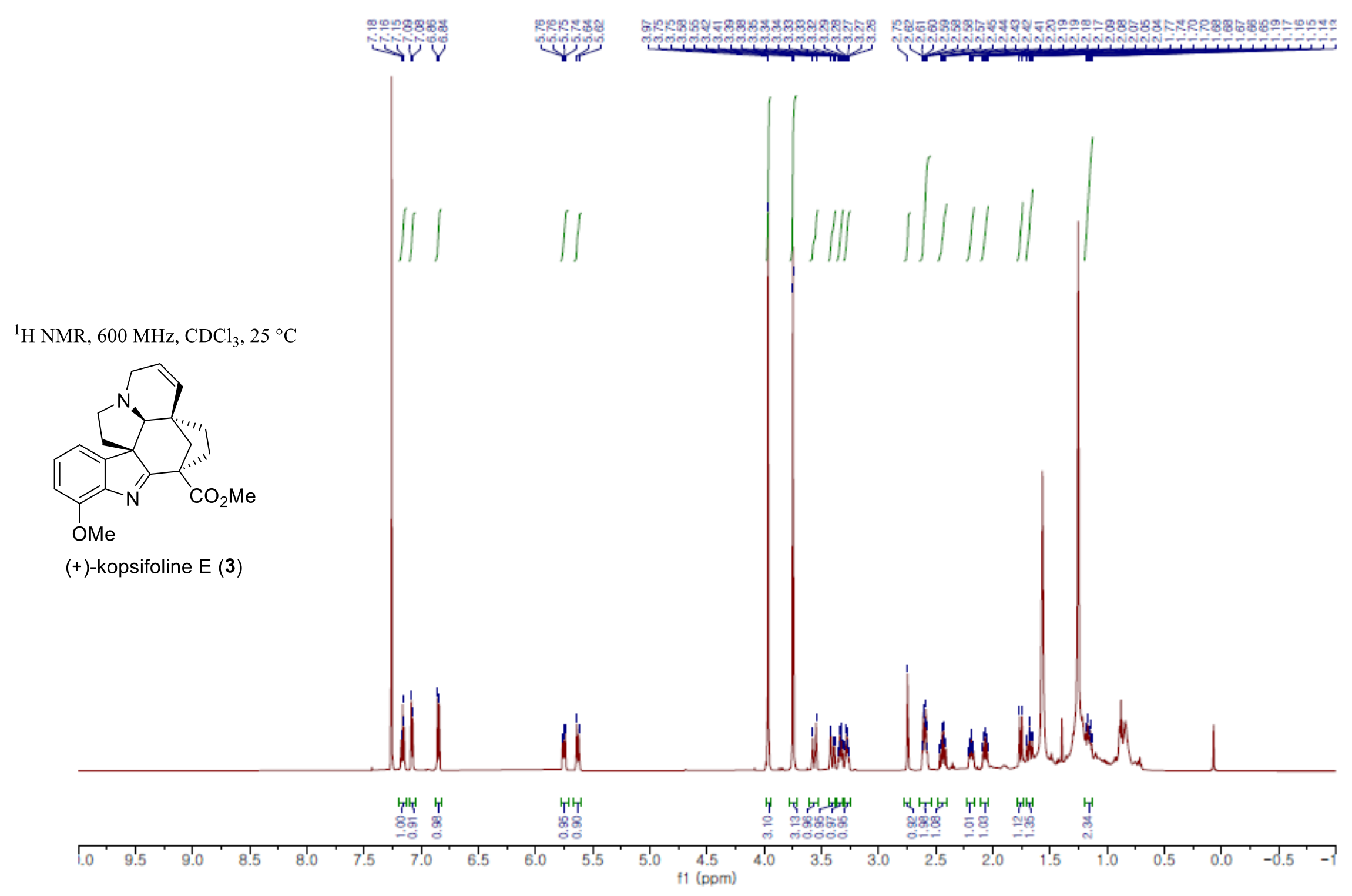




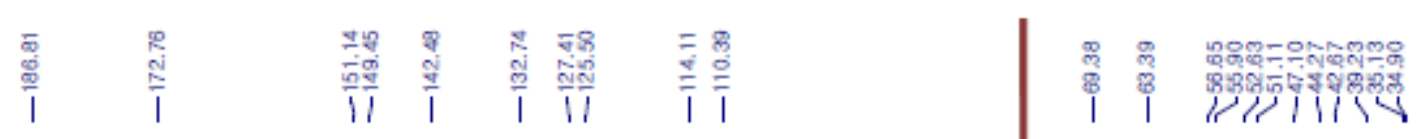

${ }^{13} \mathrm{C}$ NMR, $150.9 \mathrm{MHz}, \mathrm{CDCl}_{3}, 25^{\circ} \mathrm{C}$

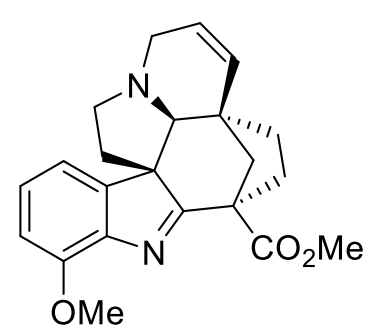

(+)-kopsifoline E (3)
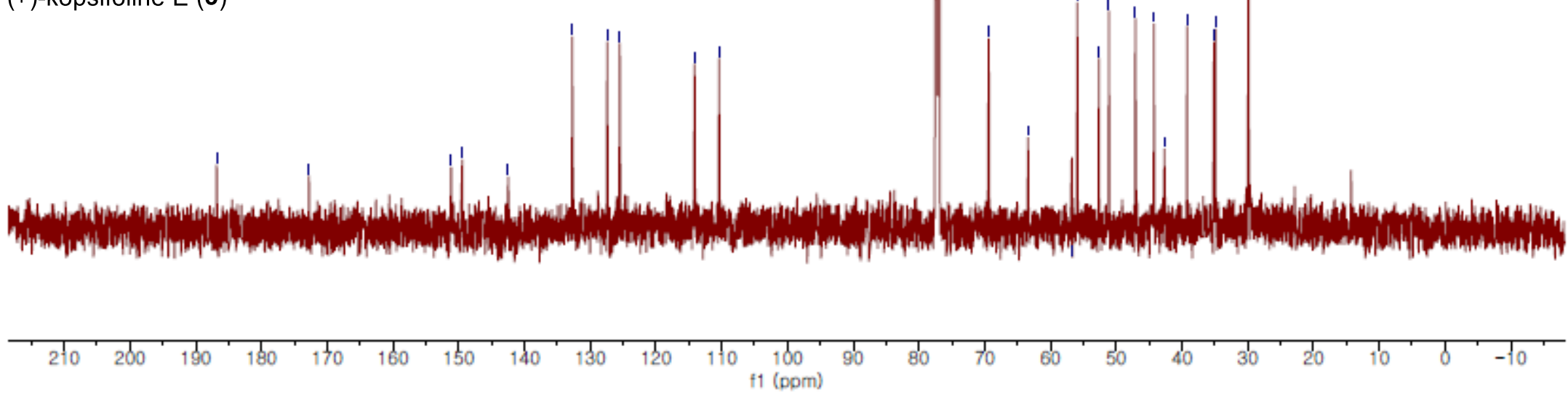


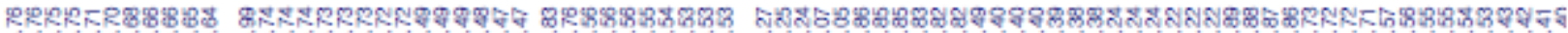

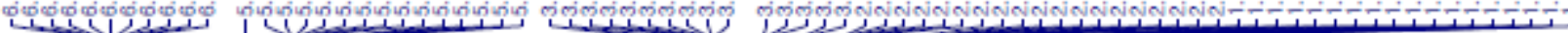

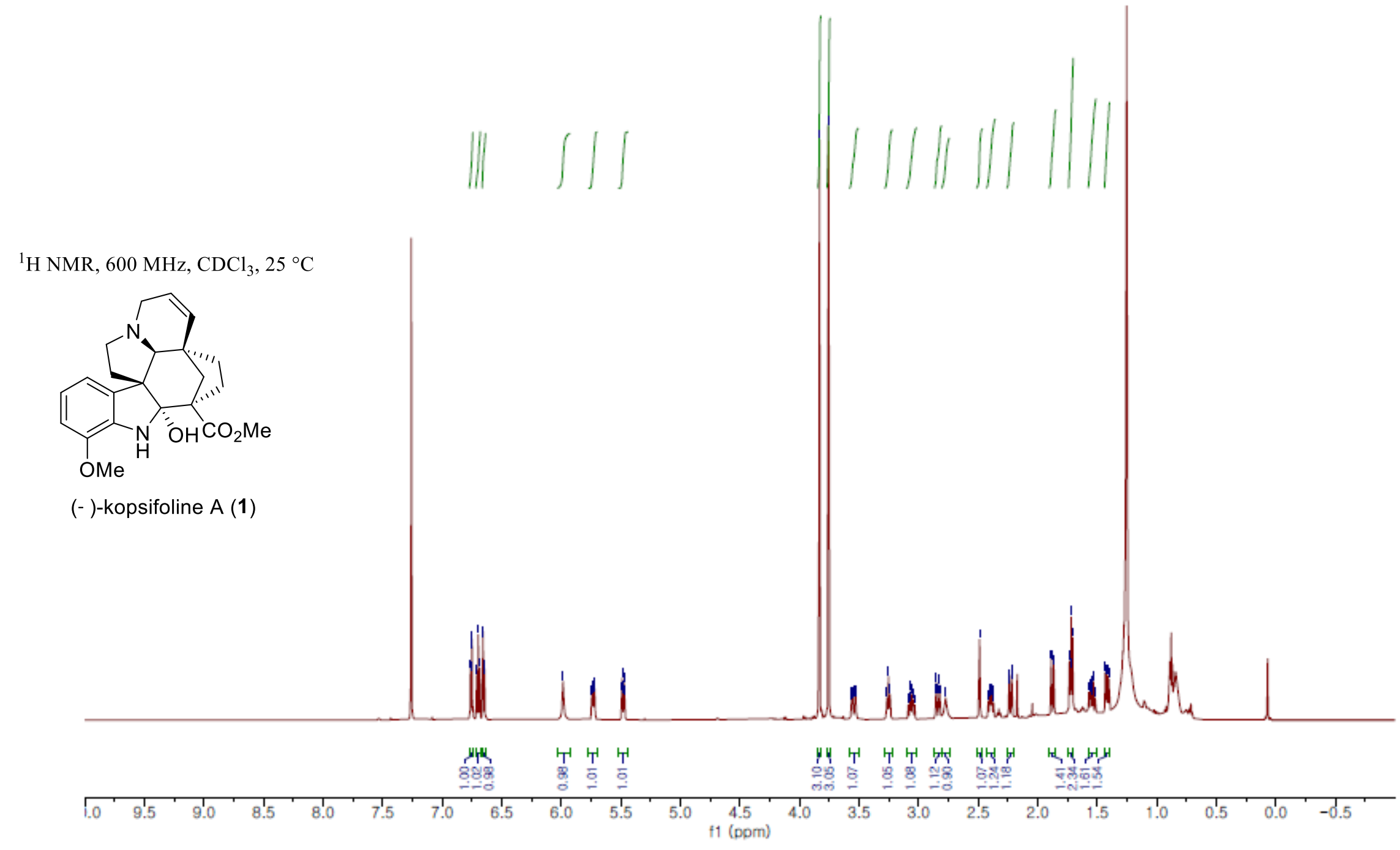




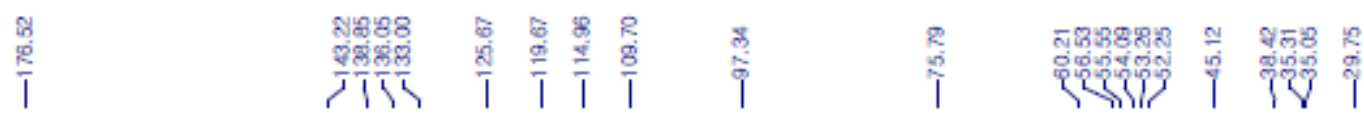

${ }^{13} \mathrm{C}$ NMR, $150.9 \mathrm{MHz}, \mathrm{CDCl}_{3}, 25^{\circ} \mathrm{C}$

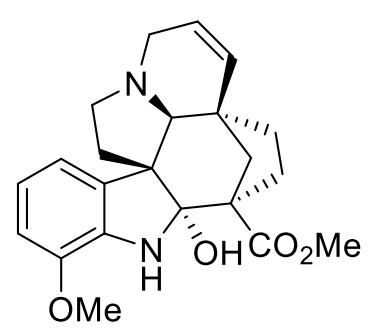

(- )-kopsifoline A (1)
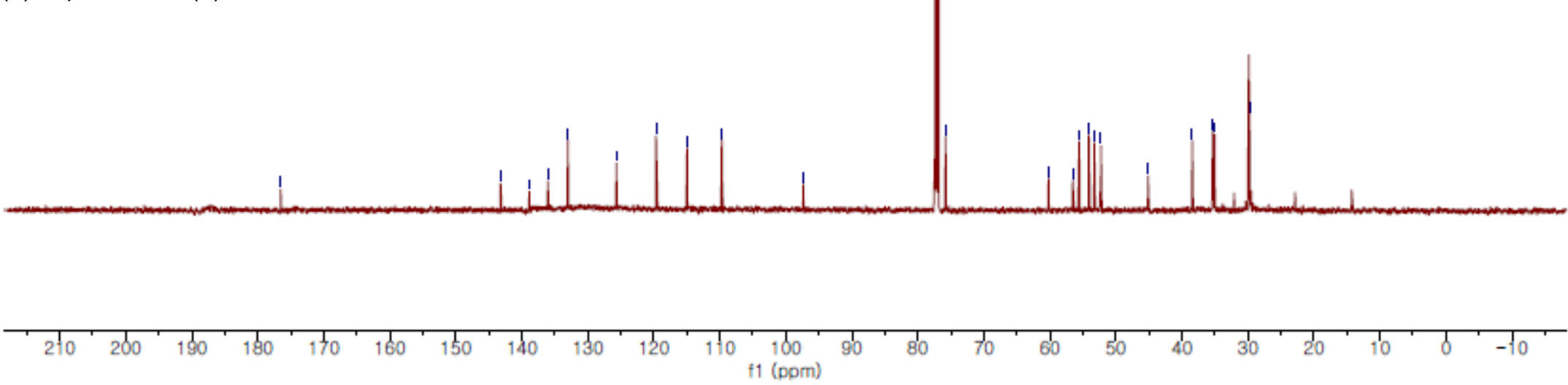